\title{
ANTINEUTRINO NEUTRAL CURRENT INTERACTONS IN MINIBOONE
}

\author{
by \\ RANJAN DHARMAPALAN \\ ION STANCU, COMMITTEE CHAIR \\ JEROME K. BUSENITZ \\ K. CLARK MIDKIFF \\ NOBUCHIKA OKADA \\ ANDREAS PIEPKE \\ SANJOY K. SARKER
}

\section{A DISSERTATION}

Submitted in partial fulfillment of the requirements

for the degree of Doctor of Philosophy in the Department of Physics and Astronomy

in the Graduate School of

The University of Alabama

TUSCALOOSA, ALABAMA 
(C)Ranjan Dharmapalan 2012

ALL RIGHTS RESERVED 


\section{Abstract}

This dissertation reports the antineutrino-nucleus neutral current elastic scattering cross section on $\mathrm{CH}_{2}$ measured by the MiniBooNE experiment located in Batavia, IL. The data set consists of 60,605 events passing the selection cuts corresponding to $10.1 \times 10^{20} \mathrm{POT}$, which represents the world's largest sample of antineutrino neutral current elastic scattering events. The final sample is more than one order of magnitude lager that the previous antineutrino NCE scattering cross section measurement reported by the BNL E734 experiment. The measurement presented in this dissertation also spans a wider range in $Q^{2}$, including the low- $Q^{2}$ regime where the cross section rollover is clearly visible.

A $\chi^{2}$-based minimization was performed to determine the best value of the axial mass, $M_{A}$ and the Pauli blocking scaling function, $\kappa$ that matches the antineutrino NCE scattering data. However, the best fit values of $M_{A}=1.29 \mathrm{GeV}$ and $\kappa=1.026$ still give a relatively poor $\chi^{2}$, which suggests that the underlying nuclear model (based largely on the relativistic Fermi gas model) may not be an accurate representation for this particular interaction.

Additionally, we present a measurement of the antineutrino/neutrino-nucleus NCE scattering cross section ratio. The neutrino mode NCE sample used in this study, corresponding

to $6.4 \times 10^{20} \mathrm{POT}$, is also the world's largest sample (also by an order of magnitude). We have demonstrated that the ratio measurement is robust, as most of the correlated errors cancel, as expected. Furthermore, this ratio also proves to be rather insensitive to variations in the axial mass and the Pauli blocking parameter. This is the first time that this ratio has been experimentally reported. We believe this measurement will aid the theoretical physics community to test various model predictions of neutrino-nucleon/nucleus interactions. 


\section{List of Abbreviations}

$\begin{array}{ll}\text { ADC } & \text { Analog-to-digital converter } \\ \text { AGS } & \text { Alternating Gradient Synchrotron } \\ \text { ATLAS } & \text { A Toroidal LHC Apparatus } \\ \text { BNB } & \text { Booster neutrino beamline } \\ \text { BNL } & \text { Brookhaven National Laboratory } \\ \text { CEBAF } & \text { Continuous Electron Beam Facility } \\ \text { CC } & \text { Charged current } \\ \text { CCQE } & \text { Neutrino-nucleon charge current quasi-elastic }\left(\nu_{l}+n \rightarrow l^{-}+p\right) \\ \text { CCPi+ } & \text { Neutrino-nucleon charge current } \pi^{+}\left(\nu_{l}+n \rightarrow l^{-}+\pi^{+}+n\right) \\ \text { CERN } & \text { European Organization for Nuclear Research } \\ \text { CKM } & \text { Cabibo-Kobayashi-Maskawa } \\ \text { CMS } & \text { Compact Muon Solenoid } \\ \text { CP } & \text { Charge Parity } \\ \text { CV } & \text { Central Value } \\ \text { CVC } & \text { Conserved Vector Current } \\ \text { DAQ } & \text { Data acquisition } \\ \text { DIF } & \text { Decay in flight } \\ \text { DOF } & \text { Degree of freedom } \\ \text { FNAL } & \text { Fermi National Accelerator Laboratory or Fermilab } \\ \text { FSI } & \text { Final state interactions } \\ \text { HARP } & \text { Hadron Production Experiment }\end{array}$




\begin{tabular}{|c|c|}
\hline HPWF & Harvard-Penn-Wisconsin-Fermilab \\
\hline $\mathrm{HV}$ & High voltage \\
\hline GEANT & Geometry and Tracking (modelling software) \\
\hline GWS & Glashow-Weinberg-Salam model \\
\hline $\mathrm{K} 2 \mathrm{~K}$ & KEK to Kamioka \\
\hline KamLAND & Kamioka Liquid Scintillator Antineutrino Detector \\
\hline KATRIN & Karlsruhe Tritium Neutrino Experiment \\
\hline LINAC & Linear accelerator \\
\hline LSND & Liquid Scintillator Neutrino Detector \\
\hline $\mathrm{MC}$ & Monte Carlo (Computer Simulated Data) \\
\hline MINUIT & CERN function minimization and error analysis package \\
\hline MiniBooNE & Mini Booster Neutrino Experiment \\
\hline MINOS & Main Injector Neutrino Oscillation Search \\
\hline MNS & Maki-Nakagawa-Sakata \\
\hline $\mathrm{NC}$ & Neutral current \\
\hline $\mathrm{NCE}$ & Neutrino-nucleon neutral current elastic \\
\hline NuMI & Neutrinos from the Main Injector \\
\hline PMT & Photomultiplier Tube \\
\hline POT & Protons on target \\
\hline$Q^{2}$ & Momentum transfer squared \\
\hline QCD & Quantum chromodynamics \\
\hline QED & Quantum electrodynamics \\
\hline SM & Standard Model \\
\hline$P E$ & Photo-electron \\
\hline PDG & Particle Data Group \\
\hline PID & Particle identification \\
\hline RFG & Relativistic Fermi gas \\
\hline
\end{tabular}




$\begin{array}{ll}\text { RENO } & \text { Reactor Experiment for Neutrino Oscillations } \\ \text { SNO } & \text { Sudbury Neutrino Observatory } \\ \text { SPE } & \text { Single photoelectron } \\ \text { T2K } & \text { Tokai to Kamioka } \\ \text { UA1 } & \text { Underground Area 1 } \\ \text { UV } & \text { Ultraviolet } \\ \text { WMAP } & \text { Wilkinson Microwave Anisotropy Probe } \\ \text { WNC } & \text { Weak neutral current } \\ \text { WS } & \text { Wrong sign }\end{array}$




\section{Acknowledgements}

First and foremost, I would like to thank my advisor, Ion Stancu. Thank you Ion, for getting me interested in experimental neutrino physics. From you, I learnt the importance of having an eye for details which is crucial for an experimentalist, and also about working hard and enjoying life at the same time.

The first part of my graduate studies was spent in Tuscaloosa, AL. It is where I spent most time in one town, after Bombay where I grew up, and deeply appreciate the culture and hospitality I experienced.

I would like to mention the Department of Physics \& Astronomy at the University of Alabama. The professors from whom I learnt most of the physics I know and with the many professors with whom I was a TA where I learnt the ropes and discovered the joy of teaching. The department office staff who were always kind and helpful in dealing with all the bureaucratic paperwork that came with being an international student. My fellow graduate students, Denis and Yujing, with whom I had a great time learning physics or discussing college football.

Being away from home was made a lot easier by my friends from Tuscaloosa (who have

graduated well before me) Vishal, Sachin, Meghna. Thank you for your friendship. The good times we had will always be cherished.

I arrived at Fermilab to begin my research and the second leg of my graduate studies. I had never imagined I would be at a world famous physics laboratory and I feel undeservedly lucky. It has been a great experience to be a part of MiniBooNE experiment. I have had the opportunity to meet many incredibly smart physicists, listen to great talks and discussions 
which kept me motivated during my research.

A special thanks to Denis Perevalov, my fellow graduate student from back in Alabama and who was my mentor in Fermilab. Thank you for helping me when I was getting started with my analysis and showing me how to navigate through the MiniBooNE analysis framework. Thank you for explaining things to me multiple times until I understood it. As I finish my measurement I have come to appreciate the work done by you to develop the NCFitter, without which my analysis would not be possible. Teppei Katori and Joe Grange, thank you for being there to talk to me whenever I had a question or was stuck during my analysis. You are both good physicists and I always learnt something from our discussions.

Many thanks to Chris Polly and Sam Zeller, MiniBooNE analysis coordinators. Chris, thank you for making time for me when I had questions or problems or ideas. Whenever I felt bogged down and lost during my research all I had to do was talk with Sam. Thank you Sam, for keeping me and other graduate students motivated by reminding us of the bigger picture and cheering every time we showed a new plot during meetings.

Zarko Pavlovic, thank you for patiently answering my questions about mutlisims, the analysis framework, condor and linux. I could not have done my ratio measurement without your help.

Richard Van De Water, MiniBooNE spokesperson, thank you for being a relentless champion of the cause. I would not have a world record antineutrino sample for my analysis without your efforts to keep the experiment running.

My stay at Fermilab was made special by the many friends I made, Jason, Josh, Vasu, Saima, Souvik, I had a great time drinking good beer, eating good food, and debating about everything under the sun, including the sun!

A shout-out to my friends from back home in India, who are now scattered all around the world. Kisalay, Ritesh, Asawari, thank you for encouraging me to apply to graduate school. My friends from my undergraduate days in Ruia college, Manish, Sunil, Ruju, Vivek.

I owe everything I have achieved to my family - my late father, my mother Lalitha and 
brothers, Visakh and Vineeth. I love you all and appreciate the sacrifices you made for me. Lastly, thank you Priya, for your love and friendship which has enriched my life more than I ever thought possible. 


\section{Contents}

$\begin{array}{ll}\text { Abstract } & \text { ii }\end{array}$

List of Abbreviations

Acknowledgements vi

List of Figures $\quad$ xiii

List of Tables $\quad$ xvi

1 Introduction $\quad 1$

1.1 The Standard Model Particles . . . . . . . . . . . . . . . . . . . . . . . 1

1.2 The Standard Model Forces _. . . . . . . . . . . . . . . . . . . . . . 4

1.3 The Electroweak Theory $\ldots \ldots \ldots \ldots$

1.4 Limitations of the Standard Model . . . . . . . . . . . . . . . . . 10

1.5 Neutrino Oscillations . . . . . . . . . . . . . . . . . . . . . . 11

1.6 Antiparticles and Antineutrinos . . . . . . . . . . . . . . . . 14

1.7 The Aim of this Dissertation . . . . . . . . . . . . . . . . . 15

1.8 Layout of this document $\ldots \ldots \ldots \ldots$

2 Neutral Current Elastic Scattering $\quad 18$

2.1 Elastic Scattering with Electrons _. . . . . . . . . . . . . . 18

2.2 Neutrino Elastic Scattering . . . . . . . . . . . . . . . . . . . 20 
2.3 Neutrino-Nucleon Neutral-Current Elastic

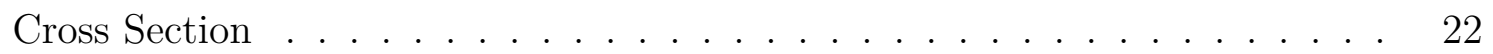

2.4 Nucleon Form Factors . . . . . . . . . . . . . . . . . . . . . . . 23

2.5 MiniBooNE Neutral Current Elastic Cross Section . . . . . . . . . . . . . 28

2.6 Previous Neutral Current Elastic Cross Section Measurements . . . . . . . . 28

2.7 MiniBooNE Neutrino Neutral Current Elastic Cross Section Measurement 31

3 The MiniBooNE Experiment $\quad 34$

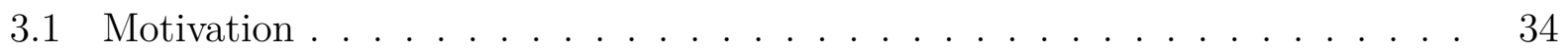

3.2 The MiniBoonE Experiment . . . . . . . . . . . . . . . . . . 37

3.3 The MiniBooNE Neutrino Beam . . . . . . . . . . . . . . . . . . . . 37

3.3.1 Primary Proton beam . . . . . . . . . . . . . . . 38

3.3.2 Secondary meson beam . . . . . . . . . . . . . . . . 39

3.3.3 Tertiary neutrino beam . . . . . . . . . . . . . . 40

3.4 The MiniBooNE Neutrino Flux . . . . . . . . . . . . . . . . . 41

3.4.1 Modelling the Primary Proton Beam and Horn . . . . . . . . . . . . 42

3.4.2 Secondary Particle Production Model . . . . . . . . . . . . . . . . 42

3.5 The MiniBooNE Detector . . . . . . . . . . . . . . . 46

3.5.1 The MiniBooNE Target-Mineral oil . . . . . . . . . . . . . . 48

3.5.2 The Photomultiplier Tubes . . . . . . . . . . . . . . . 49

3.6 Data Acquisition, Digitization and Trigger . . . . . . . . . . . . . 52

3.6.1 Data Acquisition and Digitization . . . . . . . . . . . . 52

3.6 .2 The Trigger System . . . . . . . . . . . . . . . . . 53

3.7 Calibration . . . . . . . . . . . . . . . . . . . . . 55

3.7.1 Laser Calibration System . . . . . . . . . . . . . . 56

3.7.2 Muon Calibration System . . . . . . . . . . . . . 56

3.7.3 Michel Electron Calibration . . . . . . . . . . . . . . 58

3.7.4 Neutral Pion Mass Calibration . . . . . . . . . . . . . . . 59 
3.8 The MiniBooNE Cross section model . . . . . . . . . . . . . . . . . 61

3.8 .1 Neutral Current Elastic Scattering . . . . . . . . . . . . . . . 61

3.8.2 Charged Current Elastic Scattering . . . . . . . . . . . . . . 62

3.8.3 Neutral-Current Single Pion Production . . . . . . . . . . . . . 65

3.8 .4 Nuclear effects . . . . . . . . . . . . . . . . . . . . . 65

3.8 .5 Dirt Interactions $\ldots \ldots \ldots 6$

3.9 The MiniBooNE Detector Monte Carlo . . . . . . . . . . . . . . . 66

3.9 .1 The MiniBooNE Optical Model . . . . . . . . . . . . . . . . 67

3.9.2 Simulation of Photomultiplier tube Response and Digital Acquisition 72

3.10 Event Reconstruction . . . . . . . . . . . . . . . . . . . . . 73

4 Analysis $\quad 79$

4.1 Cross section and Flux Integrated Differential Cross Section _. . . . . . . 79

4.2 Signal definition . . . . . . . . . . . . . . . . . . . . . . . 80

4.3 Analysis Cuts . . . . . . . . . . . . . . . . . . . . . . 82

4.4 Sample composition . . . . . . . . . . . . . . . . . 87

4.5 Estimation of Backgrounds $\ldots \ldots \ldots \ldots$

4.5 .1 Dirt Background . . . . . . . . . . . . . . . 88

4.5.2 Estimation of Neutrino Induced Background . . . . . . . . . . . 98

4.5 .3 The Irreducible Background . . . . . . . . . . . . . . . . . . . . . . 102

4.6 Unfolding . . . . . . . . . . . . . . . . . . . . . . . . . . . . . . . . . . 102

4.7 Measurement Uncertainties . . . . . . . . . . . . . . . . . . . . . . 104

4.8 Multisims and Unisims . . . . . . . . . . . . . . . . . 106

4.9 Error Calculation . . . . . . . . . . . . . . . . . . 107

4.9 .1 Systematic Errors . . . . . . . . . . . . . . 107

$4.9 .2 \quad$ Statistical Error . . . . . . . . . . . . . . . . . . . . 108

4.9 .3 Unfolding Errors . . . . . . . . . . . . . . . . . . 108

4.9 .4 Total Error . . . . . . . . . . . . . . . . . . . . . . 109 
4.10 The $\bar{\nu}$ Neutral Current Elastic Differential

Cross-section . . . . . . . . . . . . . . . . . 109

4.11 Antineutrino to Neutrino Neutral Current

Cross-Section Ratio . . . . . . . . . . . . . . . . . . . . . 114

4.11 .1 The Data Set . . . . . . . . . . . . . . . . . . . . 114

4.11.2 Error Estimation . . . . . . . . . . . . . . . . . . 115

4.11 .3 Result . . . . . . . . . . . . . . . . . . . . . . 118

4.12 Axial Vector Mass Measurement . . . . . . . . . . . . . . . . 120

4.12.1 Error Matrix . . . . . . . . . . . . . . . . 121

4.12 .2 Simultaneous $M_{A}$ and $\kappa$ Fit . . . . . . . . . . . . . 123

5 Conclusions $r 26$

$\begin{array}{lr}\text { A Search for a light dark matter particle at MiniBooNE } & 128\end{array}$

A.1 Introduction . . . . . . . . . . . . . . . . . . 128

A.2 MiniBooNE Neutral Current Interactions . . . . . . . . . . . . . . . . . . 129

A.3 Plan of the Project . . . . . . . . . . . . . . . . . . . 131

A.4 Conclusion . . . . . . . . . . . . . . . . . . . . . 132

$\begin{array}{ll}\text { References } & 133\end{array}$ 


\section{List of Figures}

2.1 Antineutrino quark neutral current interaction . . . . . . . . . . . . . 27

2.2 The BNL E734 cross section measurement. . . . . . . . . . . . . . . . . 29

2.3 The BNL E734 $\Delta s$ measurement. . . . . . . . . . . . . . . . 30

2.4 The MiniBoonE $\nu$ cross section. . . . . . . . . . . . . . . . 32

3.1 The LSND signal. . . . . . . . . . . . . . . . . . . . . . . 36

3.2 The MiniBooNE experimental setup. . . . . . . . . . . . . . . . . . . . . 37

3.3 The Booster neutrino beamline . . . . . . . . . . . . . . . . 38

3.4 The proton beam structure . . . . . . . . . . . . . . . . . . . . . . . 39

3.5 The MiniBooNE magnetic focussing horn . . . . . . . . . . . . . . 40

3.6 The HARP experiment $\pi^{+}$production measurements $\ldots \ldots$. . . . . . . 43

3.7 The MiniBooNE flux prediction . . . . . . . . . . . . . . . . . . . 45

3.8 A schematic of the MiniBooNE detector . . . . . . . . . . . . . 46

3.9 The signal and veto regions of the MiniBooNE detector $\ldots \ldots$. . . . . 47

3.10 A schematic of the MiniBooNE detector . . . . . . . . . . . . . 48

3.11 A PMT with support wires . . . . . . . . . . . . . . . 50

3.12 The MiniBooNE PMT support structure . . . . . . . . . . . . . . . 51

3.13 Distribution of the old and new PMTs within the MiniBooNE detector . . 51

3.14 A schematic diagram of MiniBooNE signal processing . . . . . . . . . . 53

3.15 The MiniBooNE laser calibration system . . . . . . . . . . . . 55

3.16 Muon energy calibration with the cosmic muon calibration system . . . . . 58

3.17 Michel electron calibration . . . . . . . . . . . . . . . . . . . . 59 
$3.18 \mathrm{NC} \pi^{0}$ mass calibration . . . . . . . . . . . . . . . . . 60

3.19 The geometric domain of MiniBooNE GEANT3 simulation . . . . . . . . . 67

3.20 Quantum efficiency of R5912 PMT _ . . . . . . . . . . . . . 68

3.21 Fluors in MiniBooNE oil . . . . . . . . . . . . . . . . . . 70

3.22 Extinction rate in the Marcol 7 mineral oil . . . . . . . . . . . . . . . 72

3.23 Reconstruction geometry . . . . . . . . . . . . . . . . . . . 74

3.24 Geometry of a long outgoing event . . . . . . . . . . . . . . 75

4.1 Antineutrino nucleon NCE interaction . . . . . . . . . . . . 80

$4.2 \nu_{\mu}$ CCQE candidate with decay $\ldots \ldots \ldots \ldots \ldots$

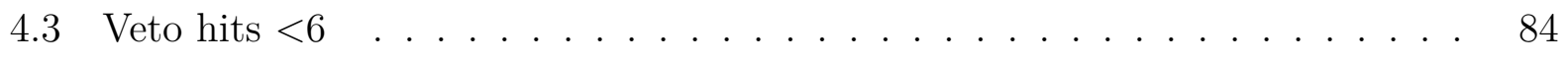

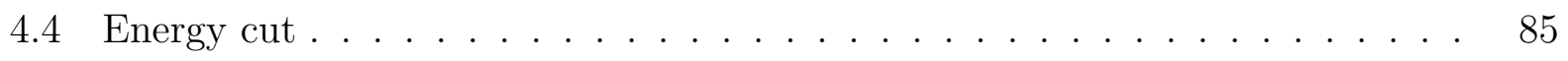

4.5 The particle identification cut . . . . . . . . . . 86

4.6 Sample composition(after analysis cuts) . . . . . . . . . . . 87

4.7 Dirt estimation using $\mathrm{Z}$ distribution $\ldots \ldots \ldots \ldots \ldots$

4.8 Dirt energy correction function from fits in the $\mathrm{Z}$ distribution . . . . . . . . 92

4.9 Dirt estimation using the $\mathrm{R}$ distribution $\ldots \ldots \ldots . \ldots . \ldots 93$

4.10 Dirt energy correction function from fits in the $\mathrm{R}$ distribution $\ldots \ldots . . .94$

4.11 Reconstructed energy distribution . . . . . . . . . . . . . . . . 95

4.12 fractions $\mathrm{f}$ and $\mathrm{g} \ldots \ldots \ldots \ldots \ldots \ldots \ldots$

4.13 Dirt energy correction function from $\mathrm{E} \ldots \ldots . \ldots . \ldots 97$

4.14 Dirt energy correction $\ldots \ldots \ldots \ldots$. . . . . . . . . . . . 98

4.15 HARP coverage . . . . . . . . . . . . . . . . . . . . . . 99

4.16 Wrong sign correction . . . . . . . . . . . . . . . . . . 101

$4.17 \bar{\nu} \mathrm{NCE}$ efficiency $\ldots \ldots \ldots \ldots \ldots$

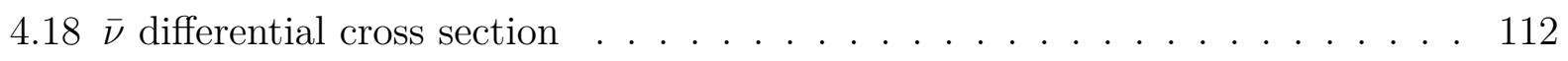

4.19 Comparing the neutrino and antineutrino mode NCE cross section . . . . . 115

4.20 Ratio of antineutrino to neutrino NCE scattering cross section . . . . . . 118 
$4.21 M_{A}$ variations compared to data . . . . . . . . . . . . . . . . . . 121

$4.22 \kappa$ variations compared to data . . . . . . . . . . . . . . . 121

4.23 Simultaneous fit to $M_{A}$ and $\kappa \ldots \ldots \ldots \ldots$. . . . . . . . . . . . . . . . . .

$4.24 \bar{\nu}$ NCE reconstructed energy spectrum . . . . . . . . . . . . . . 125

A.1 Dark matter production diagrams . . . . . . . . . . . . . . . 129

A.2 MiniBooNE dark matter exclusion plot . . . . . . . . . . . . . . . . . 130 


\section{List of Tables}

1.1 Particles in the Standard Model . . . . . . . . . . . . . . . . . . . 2

1.2 Standard Model particles (quarks) . . . . . . . . . . . . . . . . 3

1.3 Standard Model particles (leptons) . . . . . . . . . . . . . . . . 4

1.4 Fundamental forces . . . . . . . . . . . . . . . . . . . 5

1.5 Current values of neutrino oscillation mixing parameters . . . . . . . . 13

3.1 MiniBooNE trigger inputs and comparator settings . . . . . . . . . . . 54

3.2 Processes available with NUANCE I . . . . . . . . . . . . . . 63

3.3 Processes available with NUANCE II . . . . . . . . . . . . . . . . . 64

3.4 Cross-section parameters used in the MiniBooNE MC . . . . . . . . . . . . 64

4.1 Creating a dirt-enriched sample . . . . . . . . . . . . . . . 89

4.2 Antineutrino NCE cross section errors . . . . . . . . . . . . . . . 110

4.3 NCE scattering cross section errors . . . . . . . . . . . . . . 117

4.4 Normalization errors for the $M_{A}$ fit . . . . . . . . . . . . . . . . . 122 


\section{Chapter 1}

\section{Introduction}

The Standard Model of particle physics is the culmination of mankind's attempt to understand the material world. It is a framework which describes the fundamental particles in nature and the forces of interaction among them. In this chapter, a brief overview of the Standard Model is presented with some emphasis on neutrinos and the theory of weak interactions, along with an outline of the thesis.

\subsection{The Standard Model Particles}

According to the Standard Model, the universe is ultimately composed of two kinds of particles, quarks and leptons - all spin 1/2 fermions.

The quarks are presently known to have six degrees of freedom or 'flavors', viz. up $(\mathrm{u})$, down (d), strange (s), charm (c), bottom (b), and top (t). The leptons are also six

in number: the electron (e), muon $(\mu)$, tau $(\tau)$ and their corresponding neutrinos $\nu_{e}, \nu_{\mu}$, and $\nu_{\tau}$ respectively. These particles can be arranged in doublets, in increasing order of mass forming three generations, as shown in Table.1.1. It should be noted that most of the known universe is composed of the particles from the first generation. The second and third generation particles are created at particle accelerators or cosmic rays and decay rapidly into the particles of the first generation. The upper components of lepton doublets all have 


$\begin{array}{cccc}\text { Generation } \rightarrow & \text { I } & \text { II } & \text { III } \\ \text { Quarks } & \left(\begin{array}{l}u \\ d\end{array}\right) & \left(\begin{array}{l}c \\ s\end{array}\right) & \left(\begin{array}{l}t \\ b\end{array}\right) \\ \text { Leptons } & \left(\begin{array}{c}e \\ \nu_{e}\end{array}\right) & \left(\begin{array}{c}\mu \\ \nu_{\mu}\end{array}\right) & \left(\begin{array}{c}\tau \\ \nu_{\tau}\end{array}\right)\end{array}$

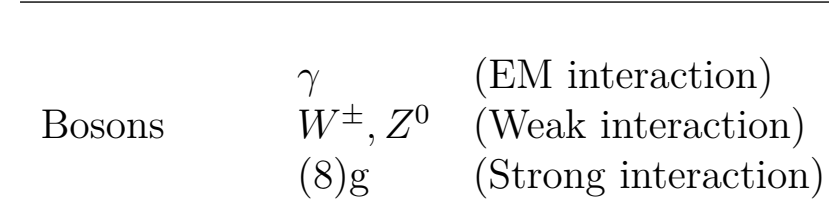

Higgs boson $H$

Table 1.1: Particles in the Standard Model. The quarks and leptons are spin $1 / 2$ particles while the bosons are spin 1.

an electric charge of -1 (in units of the absolute electron charge), while the neutrinos have charge 0 . In the quark sector the upper components have an electric charge of $+2 / 3$ while the lower components are $-1 / 3$. While the leptons can exist in isolation, the quarks, due to the fact that they posses an additional degree of freedom called "color", are only found in color neutral states called baryons ( e.g., proton $=u u d$, neutron $=u d d$, etc.), or mesons which are quark-antiquark pairs (e.g., $\pi^{+}=u \bar{d}, K^{+}=u \bar{s}$, etc.)

According to the Standard Model each of the fermions has a corresponding antiparticle. The antiparticles have the same mass and spin as their particle counterparts, but the opposite values of charge, color charge, and flavor. In the case of the neutrinos, it is still an open question if they are their own antiparticle or if the neutrino and antineutrino are distinct particles. Antiparticles are denoted by their charge labels (e.g., $e^{-}$and $e^{+}$for electrons and positrons, respectively) or a bar over the letter (e.g., $\bar{\nu}$ and $\nu$ for the neutrino and antineutrino, respectively). A list of all the quantum numbers of the quarks are given in 
Table 1.2, while Table 1.3 gives the quantum numbers of the leptons.

\begin{tabular}{cccccccc} 
Quarks & $\mathrm{Q}$ & $I_{3}$ & $S$ & $C$ & $B$ & $T$ & Mass \\
\hline \hline$u$ & $+\frac{2}{3}$ & $+\frac{1}{2}$ & 0 & 0 & 0 & 0 & $2.3_{-0.5}^{+0.7} \mathrm{MeV}$ \\
$d$ & $-\frac{1}{3}$ & $-\frac{1}{2}$ & 0 & 0 & 0 & 0 & $4.8_{-0.3}^{+0.7} \mathrm{MeV}$ \\
\hline$c$ & $-\frac{1}{3}$ & 0 & -1 & 0 & 0 & 0 & $1.275 \pm 0.025 \mathrm{GeV}$ \\
$s$ & $+\frac{2}{3}$ & 0 & 0 & +1 & 0 & 0 & $95 \pm 5 \mathrm{MeV}$ \\
$t$ & $-\frac{1}{3}$ & 0 & 0 & 0 & -1 & 0 & $173.5 \pm 0.6 \pm 0.8 \mathrm{GeV}$ \\
$b$ & $+\frac{2}{3}$ & 0 & 0 & 0 & 0 & +1 & $4.18 \pm 0.03 \mathrm{GeV}$
\end{tabular}

Table 1.2: List of quarks with their quantum numbers and mass. $Q$ : electric charge, $I_{3}:$ rd component of isospin, $S$ : strangeness, $C$ : charmness, $B$ : bottomness, $T$ : topness. These quantum numbers change their signs for anti-quarks. The values for the quark masses are from Ref. [1]

The mediators of interactions between fermions are spin 1 particles called gauge bosons. They are the photon $\gamma$ for the electromagnetic interaction, the weak bosons $W^{ \pm}$and $Z^{0}$ for the weak interaction, and eight gluons $g$ for the strong interactions. The photon and the gluons are massless, while the weak bosons are massive, $M_{W^{ \pm}} \simeq 80 \mathrm{GeV}$ and $M_{Z^{0}} \simeq 91 \mathrm{GeV}$ (throughout this thesis we use $c=1$ ).

The final particle introduced in the Standard Model is the Higgs boson H, which is a spin 0 particle. Evidence for its existence has been reported only very recently by the ATLAS and CMS experiments at the Large Hadron Collider, with a mass of about $125 \mathrm{GeV}[2,3]$. 


\begin{tabular}{cccccc} 
Leptons & $\mathrm{Q}$ & $L_{e}$ & $L_{\mu}$ & $L_{\tau}$ & Mass \\
\hline \hline$e$ & -1 & +1 & 0 & 0 & $0.511 \mathrm{MeV}$ \\
$\nu_{e}$ & 0 & +1 & 0 & 0 & $<2 \mathrm{eV}$ \\
\hline$\mu$ & -1 & 0 & +1 & 0 & $106 \mathrm{MeV}$ \\
$\nu_{\mu}$ & 0 & 0 & +1 & 0 & $<2 \mathrm{eV}$ \\
\hline$\tau$ & -1 & 0 & 0 & +1 & $1.78 \mathrm{GeV}$ \\
$\nu_{\tau}$ & 0 & 0 & 0 & +1 & $<2 \mathrm{eV}$ \\
\hline
\end{tabular}

Table 1.3: List of leptons with their quantum numbers and mass. $Q$ : electric charge, $L_{e}$ : electron number, $L_{\mu}$ : muon number, $L_{\tau}$ : tau number. These quantum numbers change their signs for anti-leptons. The values for the lepton masses are from Ref. [1]

\subsection{The Standard Model Forces}

The quarks and leptons interact among themselves and each other via the four fundamental forces known so far, namely the electromagnetic force, the weak force, the strong force and gravity. Photons are the mediators of the electromagnetic force, the weak force is mediated by the massive weak bosons, while the strong force is mediated by the gluons. All fermions also interact via the gravitational force, but the interaction is so weak that it is beyond the realm of the Standard Model. The mediator for gravitational force is the graviton, a particle with spin 2 which has not been observed to date. The electromagnetic interaction was the first to be investigated and is described by a renormalizable gauge theory with an Abelian $U(1)$ symmetry called quantum electrodynamics (QED). All fermions, with the exception of neutrinos which do not have an intrinsic electric charge, experience the electromagnetic force.

In the 1960's the weak and the electromagnetic interactions were unified into a renor- 


\begin{tabular}{cccc} 
Force & Participants & Mediator(s) & Spin \\
\hline \hline Electromagnetic & $\begin{array}{c}\text { all fermions } \\
\text { except } \nu \text { 's }\end{array}$ & $\gamma$ & 1 \\
Weak & all fermions & $W^{ \pm}, Z^{0}$ & 1 \\
Strong & only quarks \& gluons & gluons & 1 \\
Gravity & all fermions & graviton & 2 \\
& & & \\
\hline
\end{tabular}

Table 1.4: The table shows the four basic forces of interaction. Gravity is not included in the Standard Model and its mediator the graviton has not been observed.

malizable theory in the framework of a non-Abelian gauge theory with $S U(2)_{L} \otimes U(1)_{Y}$ symmetry called the electroweak standard model. The electroweak theory applies to all the fermions since they all experience the weak force. The neutrinos in particular only interact via the weak force. As this dissertation presents neutrino and antineutrino interaction measurements, we will discuss the development of the electroweak theory in some detail in the next section, $\S 1.3$.

The field theory for the strong interaction was put forth in 1970 by Gell-Mann et al. It is also a non-Abelian gauge theory with $S U(3)_{C}$ symmetry. The subscript $\mathrm{C}$ stands for the color charge, which is possessed by both the quarks and the mediator gluons. The theory, called quantum chromodynamics (QCD), describes the dynamics of quarks and gluons. A concise description of the QCD formalism can be found in Ref. [1].

Finally, the Higgs boson with spin 0 is introduced for the Higgs mechanism to work, in which the local gauge symmetry is spontaneously broken, giving rise to massive gauge bosons as well as masses to other fermions. The origin of neutrino masses remains an open issue and neutrinos have long been considered massless in the Standard Model. Recent results from neutrino oscillation experiments show that neutrinos must have non-zero masses, as we discuss in $\S 1.5$. 
To sum up, the weak and electromagnetic interactions are formulated by the gauge theory with $S U(2)_{L} \otimes U(1)_{Y}$ symmetry and the strong interaction by a gauge theory with $S U(3)_{C}$ symmetry. Hence one can describe all the interactions of elementary particles by a gauge theory with some internal symmetry $G$, which can be constructed by taking the direct product of each of the individual symmetries:

$$
G=S U(3)_{C} \otimes S U(2)_{L} \otimes U(1)_{Y} .
$$

The resultant theory is called the Standard Model of particle physics.

\subsection{The Electroweak Theory}

In 1930, Wolfgang Pauli proposed the existence of the neutrino [4] to explain the continuous spectrum of nuclear beta-decay and save the energy conservation principle from being violated. Enrico Fermi was the first to put forth a formal theory of the weak interaction including the neutrino in 1932 [5]. Using the analogy to the electromagnetic interaction he proposed an interaction equivalent to the following interaction Lagrangian,

$$
\mathcal{L}=\frac{G_{F}}{\sqrt{2}}\left(\bar{p} \gamma^{\mu} n\right)\left(\bar{e} \gamma_{\mu} \nu\right)
$$

where $\gamma^{\mu}$ are the Dirac gamma matrices and $G_{F}$ is the Fermi constant.

It is a charged current interaction in which there is a net charge transfer from the hadronic to the leptonic current. The coupling constant, $G_{F}$, is universal, i.e., it is the same for all lepton flavors. Fermi predicted a value for $G_{F}$ for the first time, which is now known to be $1.166 \times 10^{-5} \mathrm{GeV}^{-2}$. However, there is no propagator, and the currents are purely vector as in the electromagnetic interaction. Moreover, the cross section predicted is extremely small and breaks down at high energies. Hence it was clear that a modification of the theory was required. 
In 1956 T.D. Lee and C. N. Yang [6], while trying to solve the $\tau-\theta$ problem, suggested that parity may be violated in the weak sector. $\tau$ and $\theta$, both strange mesons which appeared to be identical particles decayed differently, $\tau$ to an parity odd $\operatorname{state}\left(\pi^{+}, \pi^{+}\right.$, and $\left.\pi^{-}\right)$and $\theta$ to a parity even state $\left(\pi^{+}, \pi^{0}\right.$, and $\left.\pi^{0}\right)$. One year later C.S. Wu et al. [7] carried out an experiment which conclusively established that parity was maximally violated in the nuclear beta-decay of ${ }^{60} \mathrm{Co}$.

In order to accommodate the observation of parity violation, Sudarshan and Marsak, in 1958 suggested a modification of the Fermi theory, the so called V-A model (vector minus axial vector) [8]. In the same year, Feynmann and Gell-Mann [9] independently arrived at the same conclusion; the proposed interaction Lagrangian reads now:

$$
\mathcal{L}=\frac{G_{F}}{\sqrt{2}}\left[\bar{p} \gamma^{\mu}\left(1-\gamma_{5}\right) n\right]\left[\bar{e} \gamma_{\mu}\left(1-\gamma_{5}\right) \nu\right]
$$

Meanwhile, in 1956, Reines and Cowan made the first direct observation of neutrinos [10] and in 1962 Lederman, Schwartz, Steinberg et al. showed that muon neutrino is different from an electron neutrino [11] which pointed to the existence of lepton families.

The experimental observations were presenting crucial clues about the underlying structure of the interactions, as follows:

- All particles show a $U(1)$ group invariance which was later deduced to electromagnetic interactions.

- Handedness or chirality as a unique feature of interacting particles and left-handed and right-handed particles transform differently. Parity violation in weak interactions pointed to the existence of left-handed weak-isospin doublets (or their right-handed antiparticles).

- The weak bosons must be charged - because the weak interaction is charge changing, and massive - to explain the short range of the weak force. 
- Neutrinos are exclusively left-handed and massless.

- The charged-current weak interaction is universal, which means any theory should be replicable across the three generations.

The resulting theory that incorporates all the information is called the Glashow-WeinbergSalam (GWS) model for weak interactions $[12,13,14]$. It is a non-Abelian gauge theory with $S U(2)_{L} \otimes U(1)_{Y}$ symmetry accompanied by the Higgs mechanism [15]. We define the weak hypercharge $Y$ through the Gell-Mann-Nishijima relation between electric charge $Q$ and (weak) isospin $\left(I_{3}\right)$,

$$
Q=I_{3}+\frac{1}{2} Y
$$

The introduction of Higgs mechanism leads to the spontaneous symmetry breaking $S U(2)_{L} \otimes$ $U(1)_{Y} \rightarrow U(1)_{\mathrm{EM}}$ and as a result the weak bosons acquire masses while the photon remains massless.

One may note that neutral current interactions are a theoretical consequence of the GWS model. Neutral currents were first observed in the Gargamelle experiment at CERN [16]. The discovery of $W^{ \pm}$and $Z^{0}$ at CERN in 1982 [17], at the mass range predicted by the theory was a great triumph for the model.

\section{For Leptons}

The Lagrangian for interaction of the leptons are given by [18]:

$$
\begin{aligned}
\mathcal{L}_{\text {leptons }} & =\sum_{l=e, \mu, \tau} \frac{-g}{2 \sqrt{2}}\left[\bar{\nu}_{l} \gamma^{\mu}\left(1-\gamma_{5}\right) l W_{\mu}^{+}+\bar{l} \gamma^{\mu}\left(1-\gamma_{5}\right) \nu_{l} W_{\mu}^{-}\right] \\
& -\frac{g}{4 \cos \theta_{W}} \bar{\nu}_{l} \gamma^{\mu}\left(1-\gamma_{5}\right) \nu_{l} Z_{\mu} \\
& -\frac{g}{4 \cos \theta_{W}} \bar{l}\left[L_{l} \gamma^{\mu}\left(1-\gamma_{5}\right)+R_{l} \gamma^{\mu}\left(1+\gamma_{5}\right)\right] l Z_{\mu} \\
& +g \sin \theta_{W} \bar{l} \gamma^{\mu} l A_{\mu}
\end{aligned}
$$


where the $L_{l}$ and $R_{l}$ are the chiral couplings, $g$ is the coupling constant, and $\theta_{W}$ is the weak mixing angle called the Weinberg angle.

\section{For Quarks}

The theory can be extended to include electroweak interactions of quarks by making the following observations:

- The quarks have 3 additional color degree of freedom, whereas the leptons are colorless. However since electroweak interactions are colorblind one can suppress the color index.

- All quarks are massive as opposed to the leptons, wherein the neutrinos are considered massless according to the GSW model. This introduces right-handed singlets for all the quarks, unlike in the case of leptons where the neutrinos were only left-handed.

- The quarks are fractionally charged whereas the charged leptons have unit charge. This results in the quarks having a different weak hypercharge as per the Gell-MannNishijima relation used earlier.

- With respect to the weak interaction the quark mass eigenstates are different from their flavor eigenstates and are related by the Cabibo-Kobayashi-Maskawa (CKM) matrix [1].

The Lagrangian for interaction of the quarks are given by [18]:

$$
\begin{aligned}
\mathcal{L}_{\text {quarks }}= & -\frac{g}{2 \sqrt{2}} \bar{u} \gamma^{\mu}\left(1-\gamma_{5}\right) d^{\prime} W_{\mu}^{+}-\frac{g}{2 \sqrt{2}} \bar{d}^{\prime} \gamma^{\mu}\left(1-\gamma_{5}\right) u W_{\mu}^{-} \\
& -\frac{g}{4 \cos \theta_{W}} \bar{u} \gamma^{\mu}\left[1-\frac{8}{3} \sin ^{2} \theta_{W}-\gamma_{5}\right] u Z_{\mu}+\frac{g}{4 \cos \theta_{W}} \overline{d^{\prime}} \gamma^{\mu}\left[1-\frac{4}{3} \sin ^{2} \theta_{W}-\gamma_{5}\right] d^{\prime} Z_{\mu} \\
& -\frac{2}{3} g \sin \theta_{W} \bar{u} \gamma^{\mu} u A_{\mu}+\frac{1}{3} g \sin \theta_{W} \bar{d}^{\prime} \gamma^{\mu} d^{\prime} A_{\mu} \\
& + \text { corresponding higher generation terms for }(c, s) \text { and }(t, b) .
\end{aligned}
$$


In the above equation we chose the lower isospin quarks to acquire the CKM matrix. The mixing between the mass eigenstates and the flavor eigenstates of the bottom elements of the quark doublets is represented as:

$$
\left(\begin{array}{c}
d^{\prime} \\
s^{\prime} \\
b^{\prime}
\end{array}\right)=\left(\begin{array}{ccc}
V_{u d} & V_{u s} & V_{u b} \\
V_{c d} & V_{c s} & V_{c b} \\
V_{t d} & V_{t s} & V_{t b}
\end{array}\right)\left(\begin{array}{c}
d \\
s \\
b
\end{array}\right)=\mathbf{V}\left(\begin{array}{c}
d \\
s \\
b
\end{array}\right)
$$

where $\mathbf{V}$ is the CKM matrix. The current best measurements of the magnitudes of the CKM matrix elements is [1]:

$$
\left(\begin{array}{ccc}
\left|V_{u d}\right| & \left|V_{u s}\right| & \left|V_{u b}\right| \\
\left|V_{c d}\right| & \left|V_{c s}\right| & \left|V_{c b}\right| \\
\left|V_{t d}\right| & \left|V_{t s}\right| & \left|V_{t b}\right|
\end{array}\right)=\left(\begin{array}{ccc}
0.97427 \pm 0.00015 & 0.22534 \pm 0.00065 & 0.00351_{-0.00014}^{+0.00015} \\
0.22520 \pm 0.00065 & 0.97344 \pm 0.00016 & 0.0412_{-0.0005}^{+0.0011} \\
0.00867_{-0.00031}^{+0.00029} & 0.0404_{-0.0005}^{+0.0011} & 0.999146_{-0.000046}^{+0.000021}
\end{array}\right)
$$

\subsection{Limitations of the Standard Model}

Although the Standard Model has been rigorously tested, physicists believe that it does not represent a complete picture of the universe [19]. Among the main shortcomings it lacks a theory of gravitation, and does not predict the existence of non-baryonic dark matter or dark energy.

The model has about 25 parameters which are experimentally determined. At present we do not know why these parameters have their respective values or if there are any relationships between them. Other questions include the strong CP problem and the hierarchy problem. The former is with regard to QCD and its adherence to CP-symmetry as opposed to the weak sector where it is readily broken, while the latter questions the discrepancy in the relative strengths of the fundamental forces.

There have been several experimental observations which disagree with the Standard Model predictions - like the muon anomalous magnetic moment [1], the di-muon charge 
asymmetry [20], and of particular interest to us, the phenomenon of neutrino oscillations [21] and its consequence, i.e., massive neutrinos.

\subsection{Neutrino Oscillations}

The phenomenon of neutrino oscillation provides the best explanation for a long standing mystery in experimental physics, namely the solar neutrino problem. In 1968 Davis et al. [22] performed the first in a series of experiments to measure a deficit in the number of neutrinos observed coming from the sun as compared to the solar models [23]. An analogous deficit was seen in the atmospheric neutrinos by the Kamiokande experiment in 1988 [24]. A decade later, the Super-Kamiokande experiment accounted for the atmospheric neutrino deficit by enhancing the analysis techniques to become sensitive to other flavors of neutrinos [25]. In 2002 the Sudbury Neutrino Observatory (SNO) experiment accounted for the deficit of solar electron neutrinos [26], again by designing an experiment sensitive to all active neutrino flavors. In 2003, the reactor-based KamLAND experiment showed that neutrinos undergo an oscillatory flavor conversion in vacuum in the solar $\Delta m^{2}$ range - see Eq.(1.1) for an explanation of the neutrino oscillation parameters. In addition, accelerator-based experiments (K2K [27] and MINOS [28]) have independently confirmed the atmospheric neutrino oscillations.

Neutrino oscillation is a quantum mechanical phenomenon where a neutrino of a specific lepton flavor can be measured to have a different flavor after travelling some distance in space. This is due to the fact that the neutrino flavor eigenstates $\left(\nu_{e}, \nu_{\mu}\right.$, and $\left.\nu_{\tau}\right)$ are different from their mass eigenstates denoted by $\nu_{1}, \nu_{2}$, and $\nu_{3}$. Each flavor eigenstate is a coherent superposition of the mass eigenstates, and their mixing is represented by the MakiNakagawa-Sakata (MNS) matrix - similar to the CKM matrix in the quark sector, discussed in $§ 1.3$ :

$$
\nu_{l}=\sum_{m=1}^{3} \mathbf{U}_{\mathbf{l m}} \nu_{m} \quad \text { where } \quad l=e, \mu, \tau \text {. }
$$


$\mathrm{U}$ is the unitary MNS matrix; it is a $3 \times 3$ matrix relating the three flavor eigenstates with the three mass eigenstates:

$$
\begin{aligned}
\mathbf{U} & =\left(\begin{array}{ccc}
U_{e 1} & U_{e 2} & U_{e 3} \\
U_{\mu 1} & U_{\mu 2} & U_{\mu 3} \\
U_{\tau 1} & U_{\tau 2} & U_{\tau 3}
\end{array}\right) \\
& =\left(\begin{array}{ccc}
1 & 0 & 0 \\
0 & c_{23} & s_{23} \\
0 & -s_{23} & c_{23}
\end{array}\right)\left(\begin{array}{ccc}
c_{13} & 0 & s_{13} e^{-i \delta} \\
0 & 1 & 0 \\
-s_{13} e^{i \delta} & 0 & c_{13}
\end{array}\right)\left(\begin{array}{ccc}
c_{12} & s_{12} & 0 \\
-s_{12} & c_{12} & 0 \\
0 & 0 & 1
\end{array}\right)\left(\begin{array}{ccc}
e^{\frac{i \alpha_{1}}{2}} & 0 & 0 \\
0 & e^{\frac{i \alpha_{2}}{2}} & 0 \\
0 & 0 & 1
\end{array}\right)
\end{aligned}
$$

where $\theta_{i j}$ are the three mixing angles, $c_{i j}=\cos \theta_{i j}, s_{i j}=\sin \theta_{i j}$, and $\delta$ is the CP phase. $\alpha_{1}$ and $\alpha_{2}$ are the Majorana phases which are non-zero if neutrinos are Majorana particles (§1.6). The probability of oscillation between two flavor states, in vacuum, is given by:

$$
\begin{aligned}
P_{\nu_{a} \rightarrow \nu_{b}}= & \delta_{a b}-4 \sum_{i>j} \operatorname{Re}\left(U_{a i}^{*} U_{b i} U_{a j} U_{b j}^{*}\right) \sin ^{2}\left(\Delta m_{i j}^{2} \frac{L}{4 E_{\nu}}\right) \\
& +2 \sum_{i>j} \operatorname{Im}\left(U_{a i}^{*} U_{b i} U_{a j} U_{b j}^{*}\right) \sin ^{2}\left(\Delta m_{i j}^{2} \frac{L}{2 E_{\nu}}\right)
\end{aligned}
$$

where $\delta_{a b}$ is the Kronecker delta, $U_{a i}$ is the lepton mixing matrix element with flavor index $a$ and mass eigenstate index $i, \Delta m_{i j}^{2}=m_{i}^{2}-m_{j}^{2}$ is the mass squared difference between the mass eigenstates $\nu_{i}$ and $\nu_{j}, L$ is the distance between the creation and the detection of the neutrino, and $E_{\nu}$ is the neutrino energy.

One often uses a simplified, two neutrino oscillation formalism, where one of the mass eigenstates decouples. In this case the relation between the neutrino states is described by one mixing angle, $\theta$, and one mass difference, $\Delta m^{2}=m_{2}^{2}-m_{1}^{2}$. The mixing matrix then is simply:

$$
U=\left(\begin{array}{rr}
\cos \theta & \sin \theta \\
-\sin \theta & \cos \theta
\end{array}\right),
$$


while the oscillation probability formula is reduced to

$$
P\left(\nu_{a} \rightarrow \nu_{b}\right)= \begin{cases}1-\sin ^{2} 2 \theta \sin ^{2}\left(1.27 \Delta m^{2} \frac{L}{E}\right) & \text { if } a=b \\ \sin ^{2} 2 \theta \sin ^{2}\left(1.27 \Delta m^{2} \frac{L}{E}\right) & \text { if } a \neq b .\end{cases}
$$

Here we use in standard units for neutrino oscillation experiments, namely

$$
\frac{\Delta m_{i j}^{2} L}{4 E}=1.27 \frac{\Delta m_{i j}^{2}\left(\mathrm{eV}^{2}\right) L(m)}{E(M e V)} .
$$

Several neutrino oscillations experiments observing reactor, solar, and accelerator neutrinos have provided the current best known values of oscillation parameters as given in the Particle Data Group reference [1], as summarized in Table 1.5. However, recent results from reactor

\begin{tabular}{ll} 
Parameter & best-fit $( \pm 1 \sigma)$ \\
\hline \hline$\Delta m_{21}^{2}$ & $7.58_{-0.26}^{+0.22} \times 10^{-5} \mathrm{eV}^{2}$ \\
$\left|\Delta m_{32}^{2}\right|$ & $2.35_{-0.09}^{+0.12} \times 10^{-3} \mathrm{eV}^{2}$ \\
$\sin ^{2} \theta_{12}$ & $0.306_{-0.015}^{+0.018}$ \\
$\sin ^{2} \theta_{23}$ & $0.42_{-0.03}^{+0.08}$ \\
$\sin ^{2} \theta_{13}$ & $0.0251 \pm 0.0034$ \\
\hline
\end{tabular}

Table 1.5: Current values of neutrino oscillation mixing parameters from the Particle Data Group 2012 [1].

experiments (Double-Chooz, Daya Bay, and RENO) have shown that $\theta_{13}$ is non-zero, with the following results:

Double Chooz [29]: $\sin ^{2} 2 \theta_{13}=0.109 \pm 0.030$ (stat.) \pm 0.025 (syst.),

RENO [30]: $\sin ^{2} 2 \theta_{13}=0.113 \pm 0.013$ (stat.) \pm 0.019 (syst.),

Daya Bay [31]: $\sin ^{2} 2 \theta_{13}=0.092 \pm 0.016$ (stat.) \pm 0.005 (syst.). 
Even though neutrino flavor oscillations indicate that the neutrino mass is non-zero, it does not allow us to determine the absolute scale of neutrino masses. The current best limits on neutrino mass comes from a direct electron antineutrino mass measurement from tritium beta decay by the Troitzk experiment [32];

$$
m_{\bar{\nu}_{e}}<2.05 \mathrm{eV} \text { at } 95 \% \mathrm{CL} \text {. }
$$

Stringent limits on the sum of the neutrino masses also come from cosmology as reported by the analyses of the WMAP data [33], namely:

$$
\sum m_{\nu}<2.0 \mathrm{eV} \text { at } 95 \% \mathrm{CL} .
$$

In the near future, the KATRIN experiment is expected to measure the electron antineutrino mass with sensitivity $\sim 0.20 \mathrm{eV}$.

\subsection{Antiparticles and Antineutrinos}

P.A.M. Dirac was the first to introduce the concept of an antiparticle [34]. In 1928 he made the first attempt to combine the theory of quantum mechanics with special relativity and conceived the relativistic wave equation of the electron. However, the equation permitted solutions with negative energies which defied a physical interpretation. Dirac postulated that for every particle with positive energy solution there exists a corresponding antiparticle with the same mass but opposite charge.

In 1934 W. Pauli and V. Weisskopf [35] extended the theory to include fermions. The first experimental observation of an antiparticle was made in 1932 by C. Anderson [36], who discovered positrons (anti-electrons) while studying cosmic rays using a cloud chamber. Since then, many antiparticles have been observed, confirming the idea. Finally, the present QED picture of an antiparticle was proposed by E. Stuckelberg in 1942 [37] and later formalized by 
R. Feynman in 1949 [38]. According to the Feynman-Stuckelberg interpretation the negative energy solution antiparticle is a particle propagating backward in time, while a positive energy particle is propagating forward in time, and vice-versa.

While charged particles have antiparticles with opposite charge, electrically neutral particles, like the photon or the $Z^{0}$, are self-conjugate, i.e., they are their own antiparticle. Note that both the photon and the $Z^{0}$ are bosons. Back in 1937 E. Majorana [39] postulated the possibility of self-conjugate fermions and the only possible candidates among the standard model particles are the electrically neutral neutrinos. Fermions which are self-conjugate are referred to as Majorana particles, while the other fermions are called Dirac particles. It is still an open question wether neutrinos are Majorana or Dirac particles.

\subsection{The Aim of this Dissertation}

This dissertation aims to make a high-statistics antineutrino neutral current elastic (NCE) scattering cross section measurement on carbon. The only previous measurement of this cross section was performed by the E734 experiment at the Brookhaven National Laboratory (BNL), where the antineutrino sample consisted of 1,821 events. The MiniBooNE antineutrino NCE sample, as we present in this analysis, has 60,605 candidate events with a purity of $48 \%$. This is more than one order of magnitude increase in statistics over the previous measurement.

Neutrino and antineutrino cross section information in the energy regime of MiniBooNE ( $\sim 0.8 \mathrm{GeV}$ in neutrino mode and $\sim 0.6 \mathrm{GeV}$ in antineutrino mode) is important not only for next-generation accelerator-based neutrino experiments, but also for testing the validity of various nuclear interaction models.

Recent results from the MiniBooNE neutrino charged-current quasielastic (CCQE) scattering measurement, as well as the neutrino NCE scattering measurements point to an enhancement in the cross section which has not been explained. A better agreement with the 
standard theoretical predictions is achieved if the axial mass $\left(M_{A}\right)$ is assumed to be about $20 \%$ to $30 \%$ higher than the accepted nominal value of about $1 \mathrm{GeV}$. However, there are many competing models which try to explain this discrepancy [40, 41, 42, 43, 44, 45]. A ratio measurement of the antineutrino to neutrino NCE scattering cross section will cancel many systematic errors and would be less model dependent as compared to the individual cross section measurements. In addition to the cross section measurement, this ratio should also yield a valuable input to the theoretical community to test various model predictions. This cross section ratio is also a part of the work done in this dissertation.

Finally, this dissertation also includes a $\chi^{2}$-based analysis to determine the best values of the axial mass and Pauli blocking parameter that best matches the antineutrino NCE scattering data.

\subsection{Layout of this document}

In Chapter 1 we briefly introduced the Standard Model of particle physics with an emphasis on the weak interaction. We also discussed some aspects of neutrino physics, the history, current knowledge and outstanding questions. Chapter 2 focuses on the neutral current elastic scattering interactions. We look at how scattering interactions provide us with information about the structure of matter. We go on to derive the theoretical neutrino neutral current elastic cross section formulae. We also present some previous neutral current measurements. Chapter 3 presents a description of the MiniBooNE experimental setup, in terms of both the hardware and software employed. The analysis of the antineutrino neutral current elastic scattering data in MiniBooNE is presented in Chapter 4, both in terms of the differential cross section and the underlying axial mass parameter. The analysis work done reported in Chapter 4 onwards represents the research work done as part of this dissertation, unless stated otherwise. MiniBooNE has accumulated the world's largest samples of neutral current elastic scattering events (by more than one order of magnitude), both in the neutrino and 
antineutrino mode, which makes these measurements of particular interest to the nuclear theory community. The ratio of the neutral current elastic scattering differential cross sections in neutrino and antineutrino modes is also presented in this chapter. This quantity is less dependent on the underlying parameters and has smaller systematic errors as the individual cross sections, which makes it a more robust measurement. The concluding remarks are summarized in Chapter 5, followed by an appendix which describes the possibility of using the MiniBooNE neutral current elastic scattering data to search for a light dark matter particle. 


\section{Chapter 2}

\section{Neutral Current Elastic Scattering}

In this chapter we start with the history of electron scattering experiments and how they are used to probe the structure of matter. We move on to the discovery of neutral current neutrino scattering and develop the corresponding theoretical formalism. We also discuss the various form factors employed and their origins. Finally we present some of the previous neutral current elastic scattering measurements. The formalism in this chapter follows Ref. [46]

\subsection{Elastic Scattering with Electrons}

Elastic scattering experiments have long been a favorite tool for physicists to gain insight into the fundamental structure of matter. In fact, one of the early experiments which laid the foundation of subatomic physics was the $\alpha$ scattering experiment performed by Ernest Rutherford in 1911 [47]. Rutherford observed the scattering of $\alpha$ particles off a thin gold foil target, and observed that most of the particles went through, while a few of them scattered at high angles. His conclusion that the atom was mostly empty with a positively charged nucleus, debunked the "plum pudding" model prevalent at the time, according to which electrons were embedded in a positively charged "soup".

Electrons were the first choice as projectiles in the early scattering experiments. The 
choice was based on the fact that electrons were truly point-like and the resulting Coulomb scattering was a simple and well-understood interaction. Any deviation from the Coulomb interaction prediction would indicate that the target possessed extended structure. The elastic scattering of a relativistic electron on a spinless, point-like target of mass $\mathrm{M}$ and charge $e$ is given by Mott's formulae [48, 49] as:

$$
\left(\frac{d \sigma}{d \Omega}\right)_{M o t t}=\frac{\alpha^{2}}{4 \epsilon^{2} \sin ^{4} \frac{\theta}{2}} \cdot \frac{\epsilon^{\prime}}{\epsilon} \cos ^{2} \frac{\theta}{2},
$$

where $\alpha$ is a dimensionless constant which characterizes the electromagnetic force. The energy of the incident electron, $\epsilon$, and the energy of the scattered electron, $\epsilon^{\prime}$, are related to the scattering angle $\theta$ in the laboratory frame by

$$
\epsilon^{\prime}=\frac{\epsilon}{1+\frac{2 \epsilon}{M} \sin ^{2} \frac{\theta}{2}}
$$

and the momentum transfer squared is

$$
Q^{2}=4 \epsilon \epsilon^{\prime} \sin ^{2} \frac{\theta}{2}
$$

The first evidence that the proton had a complex structure came in 1923 when O. Stern measured its anomalous magnetic moment [50], which was 2.79 times larger than that for a Dirac particle of the same mass.

R. Hofstader was the first to use electron scattering to directly probe the proton in 1955 [51] after the first high-energy electron beams (190 MeV) became available at Stanford. The experimental data differed from that of a proton possessing a point charge and point magnetic moment. He measured the charge radius of the proton to be $0.8 \mathrm{fm}$ which is close to the modern value. Hofstader's observation was understood in terms of the theoretical scattering law developed by M. Rosenbluth in 1950 [52]. This law described the composite 
effect of charge and magnetic moment scattering and is given by:

$$
\frac{d \sigma}{d \Omega}=\left(\frac{d \sigma}{d \Omega}\right)_{M o t t}\left\{F_{1}^{2}\left(Q^{2}\right)+\frac{Q^{2}}{4 M^{2}}\left[F_{2}^{2}\left(Q^{2}\right)+2\left(F_{1}\left(Q^{2}+F_{2}\left(Q^{2}\right)\right)^{2} \tan ^{2} \frac{\theta}{2}\right]\right\} .\right.
$$

The Rosenbluth formula introduces the Dirac form factor $F_{1}\left(Q^{2}\right)$ and the Pauli form factor $F_{2}\left(Q^{2}\right)$. The former represents the proton's charge and its associated Dirac magnetic moment, while the latter represents the anomalous magnetic moment of the proton. These form factors can be understood to describe the internal structure of the proton. For small four-momentum transfer squared, $Q^{2}$, they are Fourier transforms of the charge and magnetization distributions in the proton.

Theoretically, the neutron also possesses Dirac and Pauli form factors. However, the lack of a free neutron target makes measurements of the neutron form factors more difficult. Some of the difficulty was overcome by Hofstader and Yearian [53] by using a deuteron target as a carrier of neutrons and a difference method to compare the scattering from a deuteron target and a proton. These investigations first showed that the neutron could not be represented as a point nucleon and that its magnetic moment was distributed in a manner similar to that of the proton.

As more energetic electron beams became available, experiments have improved the measurement of the form factors of the nucleon. Currently, the best measurement of the proton form factors come from the Continuous Electron Beam Facility (CEBAF) at the Jefferson Laboratory [54] which employs the double polarization method - in which a polarized beam of electrons with energies up to $6 \mathrm{GeV}$ are produced and polarized observables of the proton are measured using proton polarimeters.

\section{$2.2 \quad$ Neutrino Elastic Scattering}

One of the predictions of the GWS electroweak theory, developed through the 1960's, was the existence of two charged gauge bosons, $W^{+}$and $W^{-}$, and a neutral gauge boson, $Z^{0}$, which 
act as mediators to the weak force. The charged bosons $W^{+}$and $W^{-}$were responsible for the the flavor-changing charged-current interaction, while the neutral $Z^{0}$ was responsible for the flavor conserving neutral-current interaction. The charged-current interaction mediated by $W^{ \pm}$is the classical nuclear $\beta$-decay which led to the discovery of the weak force and has been widely studied, but the $Z^{0}$ mediated weak neutral current (WNC) emerges as a result of the theoretical considerations of the GWS model; until then there was no search for experimental evidence for weak neutral current iterations.

Soon after the theoretical foundation was laid, the search began for $Z^{0}$ mediated neutral current interactions. The first observation of WNC was made in 1973 by the Gargamelle experiment [16] at CERN. Gargamelle was a giant bubble chamber detector which observed a single antineutrino-electron neutral current elastic interaction:

$$
\bar{\nu}_{\mu}+e^{-} \rightarrow \bar{\nu}_{\mu}+e^{-} .
$$

Later more WNC events were observed which were neutrino-nucleon neutral current deep inelastic events,

$$
\bar{\nu}_{\mu}+N \rightarrow \bar{\nu}_{\mu}+X,
$$

where $X$ is a hadronic final state. The HPWF (Harvard-Penn-Wisconsin-Fermilab) experiment at FNAL [55] also saw deep inelastic neutrino-nucleon neutral current events which confirmed the Gargamelle result.

The immediate fallout of the observation of WNC was the prediction of the mass value of the weak gauge vector boson $W^{ \pm}$on the basis of the GSW model combined with the first measurements of the weak mixing angle $\theta_{W}$. This led to the idea of building a protonantiproton collider at CERN to search for the mediators of the weak iteration.

In 1982 the $W^{ \pm}$and $Z^{0}$ were discovered by the UA1 experiment [17] which was a great triumph for the standard model of weak interaction. The current values for the the masses 
of the weak bosons are [1]:

$$
M_{W^{ \pm}}=80.399 \pm 0.023 \mathrm{GeV} \quad \text { and } \quad \mathrm{M}_{\mathrm{Z}^{0}}=91.1876 \pm 0.0021 \mathrm{GeV}
$$

\subsection{Neutrino-Nucleon Neutral-Current Elastic Cross Section}

We now turn our attention to the theoretical aspects of WNC, specifically to the neutrinonucleon neutral current elastic interaction. This analysis aims to measure the interaction in which the incoming antineutrino interacts with the quarks in the nucleus of the $\mathrm{C}$ or $\mathrm{H}$ atom (since the MiniBooNE mineral oil target is mainly $\mathrm{CH}_{2}$ ). Following the theory developed in

$\S 1.3$, the most general Lagrangian for neutrino-hadron neutral current can be written as [56]:

$$
\mathcal{L}_{N C}^{\nu H}=\sum_{l=e, \mu, \tau}-\frac{G_{F}}{\sqrt{2}} \bar{\nu}_{l} \gamma^{\mu}\left(1-\gamma_{5}\right) \nu_{l} J_{\mu}^{H},
$$

Assuming contributions from not only the valence quarks $u$ and $d$, but also from the sea quarks $(s, c, t, b)$ we can expand the hadronic current as [56]:

$$
\begin{aligned}
J_{\mu}^{H} & =\sum_{q}\left[\varepsilon_{L}^{q} \bar{q} \gamma^{\mu}\left(1+\gamma_{5}\right) q\right]+\left[\varepsilon_{R}^{q} \bar{q} \gamma^{\mu}\left(1+\gamma_{5}\right) q\right] \\
& =\sum_{q} \bar{q} \gamma^{\mu}\left(g_{V}^{q}-g_{A}^{q} \gamma_{5}\right) q
\end{aligned}
$$

where the sum extends over the quark flavors (i.e., $q=u, d, s, c, t, b)$. The vector and axialvector couplings $g_{V, A}^{q}$ are related to the chiral couplings $\varepsilon_{L, R}^{q}$ by:

$$
\begin{aligned}
g_{V}^{q} & =\varepsilon_{L}^{q}+\varepsilon_{R}^{q}, \\
g_{A}^{q} & =\varepsilon_{L}^{q}-\varepsilon_{R}^{q} .
\end{aligned}
$$

Further, even though it is not rigorously proven, an absence of experimental evidence of 
flavor-changing neutral current effects allow us to assume that:

$$
\begin{aligned}
& \varepsilon_{L, R}^{b}=\varepsilon_{L, R}^{s}=\varepsilon_{L, R}^{d}, \\
& \varepsilon_{L, R}^{t}=\varepsilon_{L, R}^{c}=\varepsilon_{L, R}^{u} .
\end{aligned}
$$

Experiments suggest that the contributions from the heavier quarks are negligible except from the strange quark sea which may add to the proton spin giving, rise to the so-called "proton spin crisis" [57].

We are now in a position to write the matrix element squared for the neutrino-nucleon neutral current interaction as:

$$
\mathcal{M}=\frac{g^{2}}{2 M_{Z}^{2} \cos ^{2} \theta_{W}}\left[\bar{\nu} \gamma_{\mu}\left(1-\gamma_{5}\right) \nu\right]\left\langle N_{f}\left|J_{Z}^{\mu}\right| N_{i}\right\rangle
$$

The leptonic current in the square brackets is exactly calculable, but the hadronic current is quite complex due to strong interactions inside the nucleon. The matrix element of the neutral weak hadronic current between the nucleon states can be written as:

$$
\left\langle N_{f}\left|J_{Z}^{\mu}\right| N_{i}\right\rangle=\left\langle N_{f}\left|F_{1}^{Z}\left(Q^{2}\right)+F_{2} Z\left(Q^{2}\right) \frac{i \sigma^{\mu \nu} q_{\nu}}{2 M_{N}}+F_{A}^{Z}\left(Q^{2}\right) \gamma^{\mu} \gamma_{5}\right| N_{i}\right\rangle
$$

where the vector form factors, $F_{1}^{Z}\left(Q^{2}\right)$ and $F_{2}^{Z}\left(Q^{2}\right)$, are the Dirac and Pauli form factors which are taken from electron scattering experiments as discussed in $\S 2.1 . F_{A}^{Z}\left(Q^{2}\right)$ is the additional axial vector weak neutral current form factor, thus following the $V-A$ form of the weak interaction.

\subsection{Nucleon Form Factors}

In scattering theory form factors provide a link between experimental observation and theoretical analysis. It is usually a multiplicative factor $F\left(Q^{2}\right)$ - where $Q^{2}$ is the momentum 
transfer squared. To be precise, the form factor can be shown to be the Fourier transform of the charge distribution within the nucleon.

In the present case the precise structure of the nucleon is not well known, and the nucleon form factors which are derived from various experiments - such as electron scattering data - encompass the best description of the nucleon as seen by the incoming neutrino.

In order to arrive at the nucleon form factors we start with the expression for the neutral weak current,

$$
J^{Z}=\frac{1}{2} \tau_{3} J-2 \sin ^{2} \theta_{W} J^{E M}
$$

where $J^{Z}$ is the weak neutral current, $J$ is the weak charged current, $J^{E M}$ is the electromagnetic current, $\theta_{W}$ is the Weinberg angle, and $\tau_{3}=\operatorname{diag}(1,-1)$. From the above equation we can write the neutral current form factors as:

$$
\begin{aligned}
& F_{i}^{Z}=\left(F_{i}-F_{i}^{s}\right) \frac{\tau_{3}}{2}-2 \sin ^{2} \theta_{W} F_{i}^{E M}, \quad i=1,2 \\
& F_{A}^{Z}=\left(F_{A}-F_{A}^{s}\right) \frac{\tau_{3}}{2} .
\end{aligned}
$$

where $F_{1,2}^{Z}$ is the weak vector form factor and $F_{A}^{Z}$ is the weak axial form factor. The charged current form factor $F_{1,2}$ is generalized to have an isoscalar part $F_{1,2}^{s}$ and $F_{1,2}^{E M}$ is the electromagnetic form factor.

The conserved vector current (CVC) hypothesis [58] allows us to relate is $F_{1}^{Z}$ and $F_{2}^{Z}$ to the electromagnetic form factors for protons and neutrons which are better measured (see $\S 2.1)$

$$
\begin{aligned}
F_{i}^{Z}= & \left(\frac{1}{2}-\sin ^{2} \theta_{W}\right)\left[F_{i}^{E M, p}-F_{i}^{E M, n}\right] \tau_{3} \\
& -\sin ^{2} \theta_{W}\left[F_{i}^{E M, p}-F_{i}^{E M, n}\right]-\frac{1}{2} F_{i}^{s}, \quad i=1,2 . \\
F_{A}^{Z}= & \frac{\tau_{3}}{2} F_{A}-\frac{1}{2} F_{A}^{s},
\end{aligned}
$$

where we have used the relation:

$$
F_{i}=F_{i}^{E M, p}-F_{i}^{E M, n}, \quad \text { where } i=1,2 .
$$


The superscripts $p$ and $n$ in the above equations stand for proton and neutron, respectively.

One can combine the Dirac and Pauli electromagnetic form factors to define the Sachs form factors,

$$
\begin{aligned}
& G_{E}=F_{1}^{E M}-\tau F_{2}^{E M}, \\
& G_{M}=F_{1}^{E M}+F_{2}^{E M} .
\end{aligned}
$$

According to scattering theory, the electric charge density distribution and the current density distributions are derived from the three dimensional Fourier transform of $G_{E}\left(Q^{2}\right)$ and $G_{M}\left(Q^{2}\right)$, respectively. In terms of the momentum transferred $\left(Q^{2}\right)$ we can express the form factors as:

$$
\begin{aligned}
& F_{1}^{E M}\left(Q^{2}\right)=\frac{G_{E}(0)+\frac{Q^{2}}{4 M^{2}} G_{M}(0)}{\left(1+\frac{Q^{2}}{M^{2}}\right)\left(1+\frac{Q^{2}}{M^{2}}\right)^{2}}, \\
& F_{2}^{E M}\left(Q^{2}\right)=\frac{G_{M}(0)-G_{E}(0)}{\left(1+\frac{Q^{2}}{M^{2}}\right)\left(1+\frac{Q^{2}}{M^{2}}\right)^{2}},
\end{aligned}
$$

where we use the fact that the experiments suggest that the $Q^{2}$ dependence is consistent with the form factors having a dipole form,

$$
G_{E}^{Q^{2}}=\frac{G_{E}(0)}{\left(1+\frac{Q^{2}}{M_{V}^{2}}\right)}, \quad G_{M}^{Q^{2}}=\frac{G_{M}(0)}{\left(1+\frac{Q^{2}}{M_{V}^{2}}\right)}
$$

The vector mass, $M_{V}=0.843 \mathrm{GeV}$, is the same for both electric and magnetic form factors. In the $Q^{2} \rightarrow 0$ limit the form factors are normalized by the following conditions which give the electric charge of the nucleon and the anomalous magnetic moment, respectively:

$$
\begin{aligned}
G_{E}^{p}(0) & =1, \\
G_{E}^{n}(0) & =0, \\
G_{M}^{p}(0) & =1.793, \\
G_{M}^{n}(0) & =-1.91,
\end{aligned}
$$


where again the superscripts $p$ and $n$ denote the proton and neutron, respectively. The axial isovector form factor is given by

$$
F_{A}=\frac{F_{A}(0)}{\left(1+\frac{Q^{2}}{M_{A}^{2}}\right)^{2}}
$$

where $F_{A}(0)=g_{A}=1.2671$ is measured precisely from neutron beta decay.

The axial vector mass $M_{A}$ is a bit of mystery. Previous measurements, which were mostly deuterium-based bubble chamber experiments, set a value of $M_{A}=1.026 \pm 0.021 \mathrm{GeV}$. Recent experiments on nuclear targets have reported $M_{A}$ values which are approximately 20-30\% higher. MiniBooNE, using the charged-current quasi-elastic (CCQE) channel reported a value of $M_{A}=1.35 \pm 0.17 \mathrm{GeV}[59]$. Furthermore, in the neutrino mode NCE analysis a value of $M_{A}=1.39 \pm 0.11 \mathrm{GeV}$ was reported [60], consistent with that from the CCQE channel.

Finally, the isoscalar form factors, $F_{1}^{s}$ and $F_{2}^{s}$, are contributions of the strange quarks to the electric charge and to the magnetic moment of the nucleon, and $F_{A}^{s}$ is the strange quark contribution to the nucleon spin. They are usually expressed in the dipole form, analogous to the isovector form factors discussed above:

$$
\begin{aligned}
& F_{1}^{s}\left(Q^{2}\right)=\frac{F_{1}^{s}(0)}{(1+\tau)\left(1+\frac{Q^{2}}{M_{V}^{2}}\right)^{2}} \\
& F_{2}^{s}\left(Q^{2}\right)=\frac{F_{2}^{s}(0)}{(1+\tau)\left(1+\frac{Q^{2}}{M_{V}^{2}}\right)^{2}} \\
& F_{A}^{s}\left(Q^{2}\right)=\frac{F_{A}^{s}(0)}{\left(1+\frac{Q^{2}}{M_{A}^{2}}\right)^{2}}
\end{aligned}
$$

where $M_{A}$ and $M_{V}$ are assumed to have the same form as in the isovector case. For the 
limiting case of zero momentum transfer we have

$$
\begin{aligned}
& F_{1}^{s}(0)=-\frac{1}{6}\left\langle r_{s}^{2}\right\rangle, \\
& F_{2}^{s}(0)=\mu_{s}, \\
& F_{A}^{s}(0)=\Delta s .
\end{aligned}
$$

where $\left\langle r_{s}^{2}\right\rangle$ is the strange radius, $\mu_{s}$ is the strange magnetic moment of the nucleon, and $\Delta s$ is the strange quark contribution to the nucleon spin.

Parity violating electron scattering experiments give us a measure of $F_{1}^{s}$ and $F_{2}^{s}$ and recent results show these to be consistent with $0 . \Delta s$ can be extracted form neutrino-nucleus NCE experiment. The MiniBooNE neutrino mode NCE analysis reported a $\Delta s$ consistent with 0.

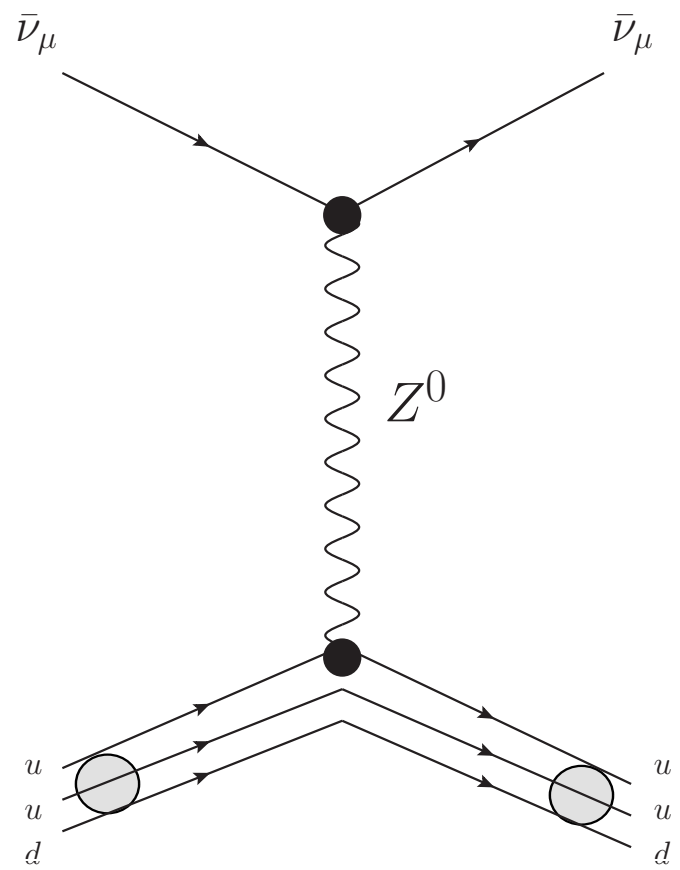

(a)

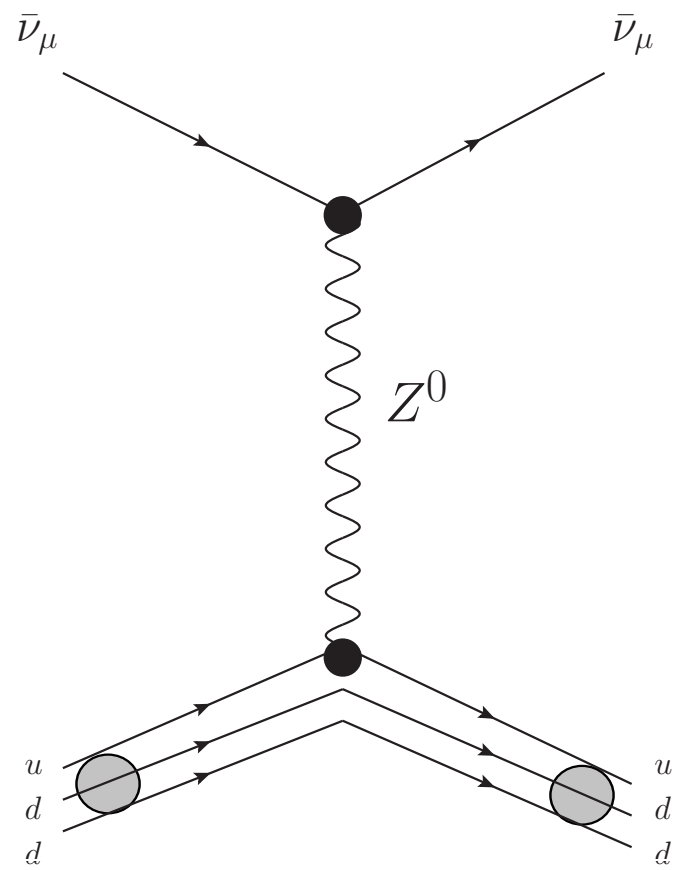

(b)

Figure 2.1: $\quad \bar{\nu}$ quark level neutral current interaction. Plot (a) shows the the $\bar{\nu}$-proton interaction and (b) shows the interaction with the neutron. 


\subsection{MiniBooNE Neutral Current Elastic Cross Section}

Using the vector and axial vector form factors the NCE neutrino nucleon cross section can be written in the formalism of Llewellyn-Smith [61],

$$
\frac{d \sigma}{d Q^{2}}=\frac{G_{F}^{2} Q^{2}}{2 \pi E_{\nu}^{2}}\left[A\left(Q^{2}\right) \pm B\left(Q^{2}\right)+C\left(Q^{2}\right) W^{2}\right]
$$

where the + sign in front of the $B\left(Q^{2}\right)$ term is for neutrinos and - sign is for antineutrinos. $G_{F}$ is the Fermi constant, $W=4 E_{\nu} / M_{N}-Q^{2} / M_{N}^{2}$ with $M_{N}$ representing the nucleon mass, and $E_{\nu}$ is the energy of the incoming neutrino. The functions $A\left(Q^{2}\right), B\left(Q^{2}\right)$ and $C\left(Q^{2}\right)$ contain the nucleon form factors:

$$
\begin{aligned}
& \left.A\left(Q^{2}\right)=\frac{1}{4}\left[\left(F_{A}^{Z}\right)^{2}(1+\tau)-\left(\left(F_{1}^{Z}\right)^{2}-\tau\left(F_{2}^{Z}\right)^{2}\right)\right)(1-\tau)+4 \tau F_{1}^{Z} F_{2}^{Z}\right], \\
& B\left(Q^{2}\right)=\frac{1}{4} F_{A}^{Z}\left(F_{1}^{Z}+F_{2}^{Z}\right), \\
& C\left(Q^{2}\right)=\frac{M_{N}^{2}}{16 Q^{2}}\left[\left(F_{A}^{Z}\right)^{2}+\left(F_{1}^{Z}\right)^{2}+\tau\left(F_{2}^{Z}\right)^{2}\right],
\end{aligned}
$$

where $\tau=Q^{2} / 4 M_{N}^{2} . F_{1}, F_{2}$, and $F_{A}$ are the nucleon Dirac, Pauli, and axial form factors, and may be different for protons and neutrons.

\subsection{Previous Neutral Current Elastic Cross Section Mea- surements}

The first neutrino-nucleon NCE scattering experiments were conducted in 1976 by the Columbia-Illinois-Rockefeller and HPWF experiments. The BNL E734 experiment was the first experiment to measure the NCE scattering cross section on protons in both $\nu$ and $\bar{\nu}$ mode with high statistics [62]. BNL E734 was a 170 ton high-resolution target detector on the BNL AGS source. The proton beam had an energy of $28 \mathrm{GeV}$ and resulted in neutrinos and antineutrinos of mean energy $1.3 \mathrm{GeV}$ and $1.2 \mathrm{GeV}$ respectively. The NCE flux-averaged 
cross section is shown in Fig. 2.2. The experiment obtained a total of 1,686 neutrino proton scattering events and 1,821 antineutrino proton scattering events.

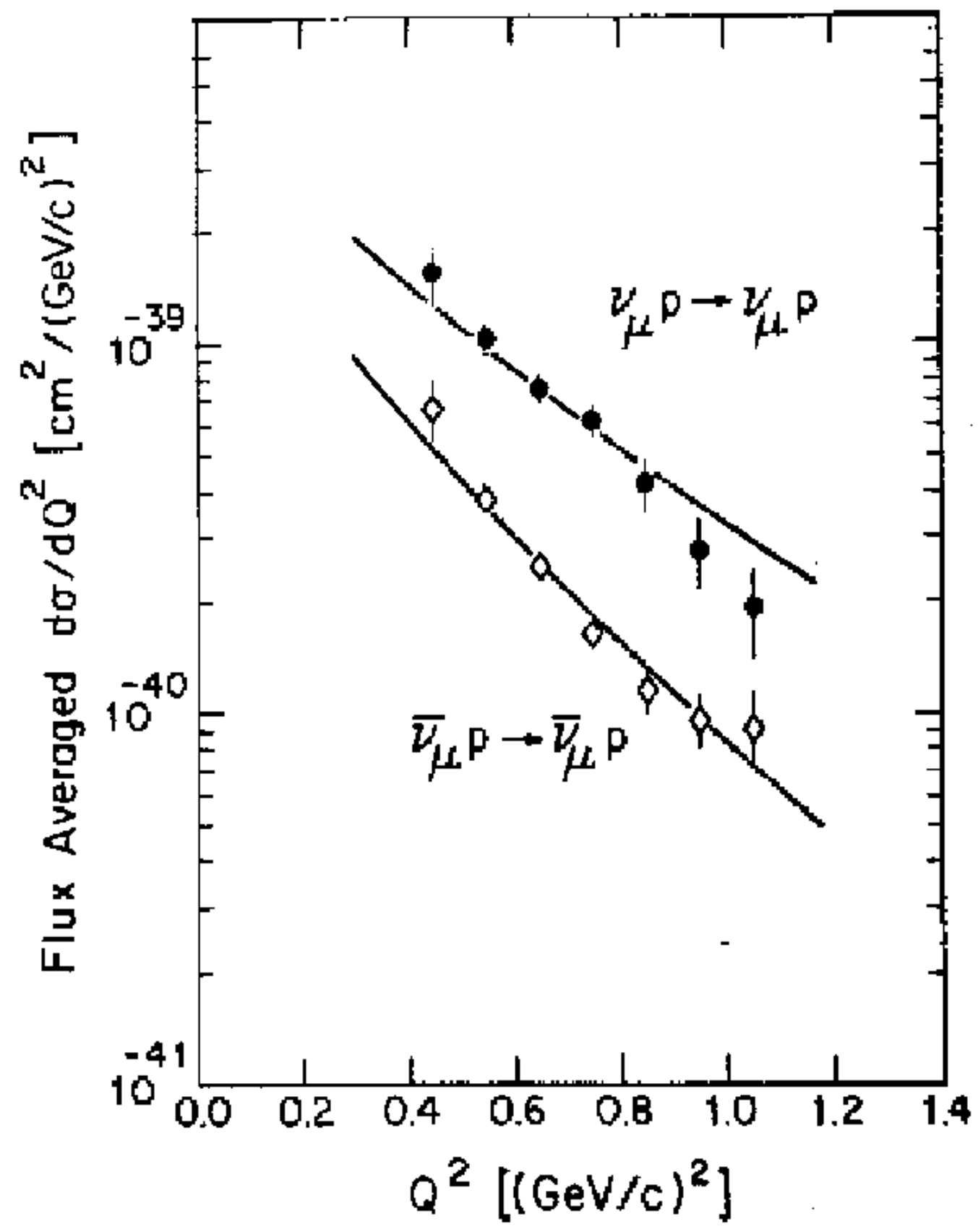

Figure 2.2: Neutrino and antineutrino cross section measurements as reported by the BNL E734 experiment - figure from Ref. [62].

The BNL E734 experiment also measured the parameter $\Delta s$, or to be precise, using their 
NCE cross section data they obtained an allowed region for $\eta$ and $M_{A}$, where $\eta$ is directly related to $\Delta$, namely $\eta=-\Delta s / g_{A}$. The allowed regions at the $67 \%$ and $90 \%$ confidence levels, are shown in Fig. 2.3.

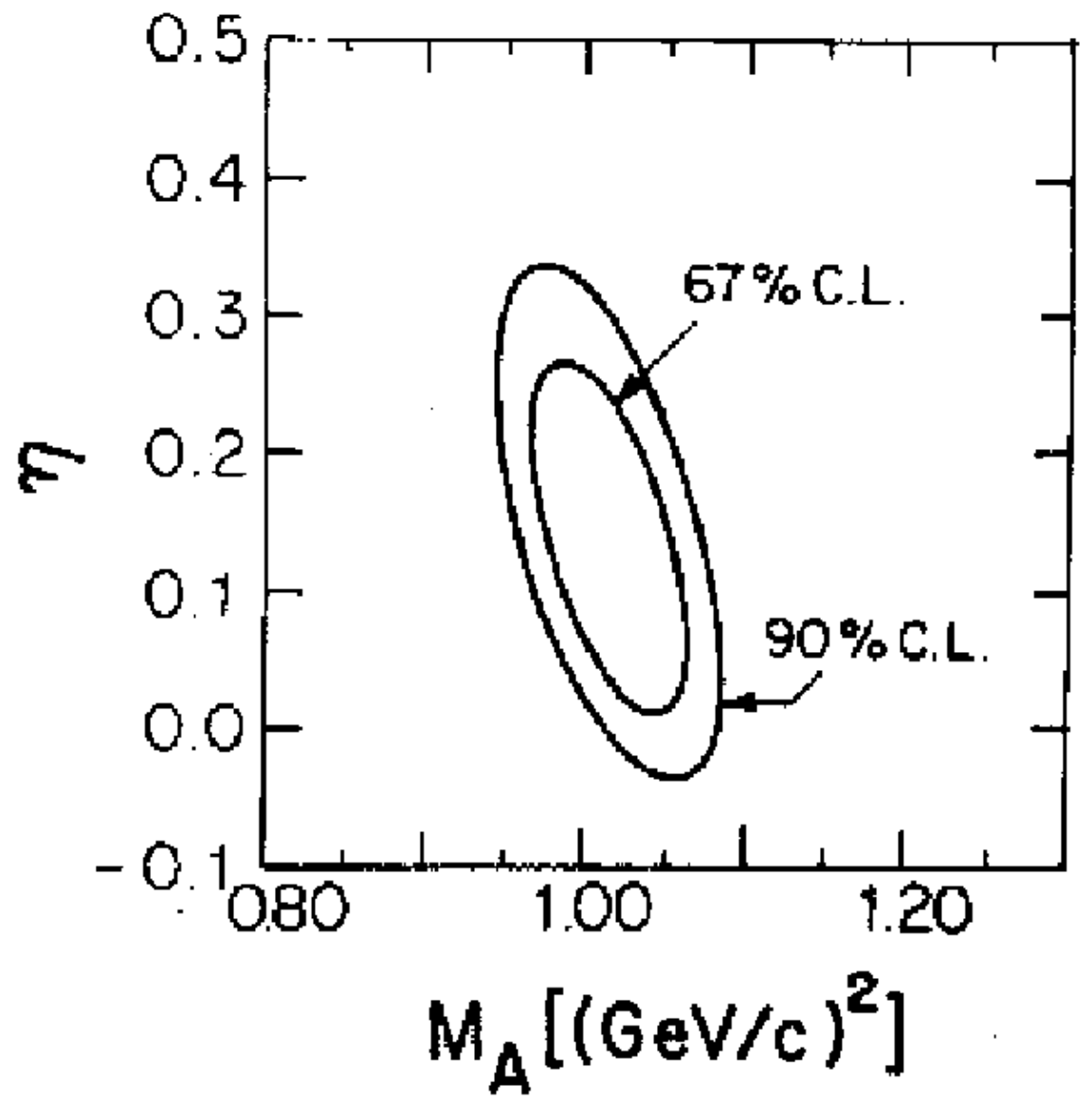

Figure 2.3: BNL E734 allowed region for $M_{A}$ and $\eta\left(\eta=-\Delta s / g_{A}\right)$ - figure from Ref. [62]. 


\subsection{MiniBooNE Neutrino Neutral Current Elastic Cross Section Measurement}

MiniBooNE collected $6.46 \times 10^{20}$ protons on target $(\mathrm{POT})$ running in neutrino mode, resulting in 94,531 NCE events which passed the NCE selection criteria. This is the largest NCE sample collected to date, with an efficiency of $35 \%$ and purity of $65 \%$. Here efficiency refers to the detector efficiency in discerning NCE events, and purity refers to the NCE composition of the sample.

After background subtraction MiniBooNE reported [60] a flux-averaged differential cross section in terms of momentum transferred squared to the nucleon, $Q_{Q E}^{2}$, as illustrated in Fig. 2.4. Note that in MiniBooNE $Q_{Q E}^{2}$ is the total kinetic energy of the outgoing nucleons in the interaction, assuming the target nucleon to be at rest:

$$
Q_{Q E}^{2}=2 M_{N} \sum_{i} T_{i}=2 M_{N} T
$$

where $M_{N}$ is the nucleon mass, and $T$ is the sum of the kinetic energies of the final state nucleons. In MiniBooNE, $T$ is proportional to the total visible charge recorded by the photomultiplier tubes (PMTs) of the detector. This makes the MiniBooNE NCE cross section measurement less sensitive to final state interactions (FSI) as compared to tracking detectors - where low energy outgoing nucleons may not be fully visible.

Finally, it should be noted that the NCE scattering discussed here is off both bound nucleons in carbon and free nucleons in the hydrogen atom of the target mineral oil $\left(\mathrm{CH}_{2}\right)$. 


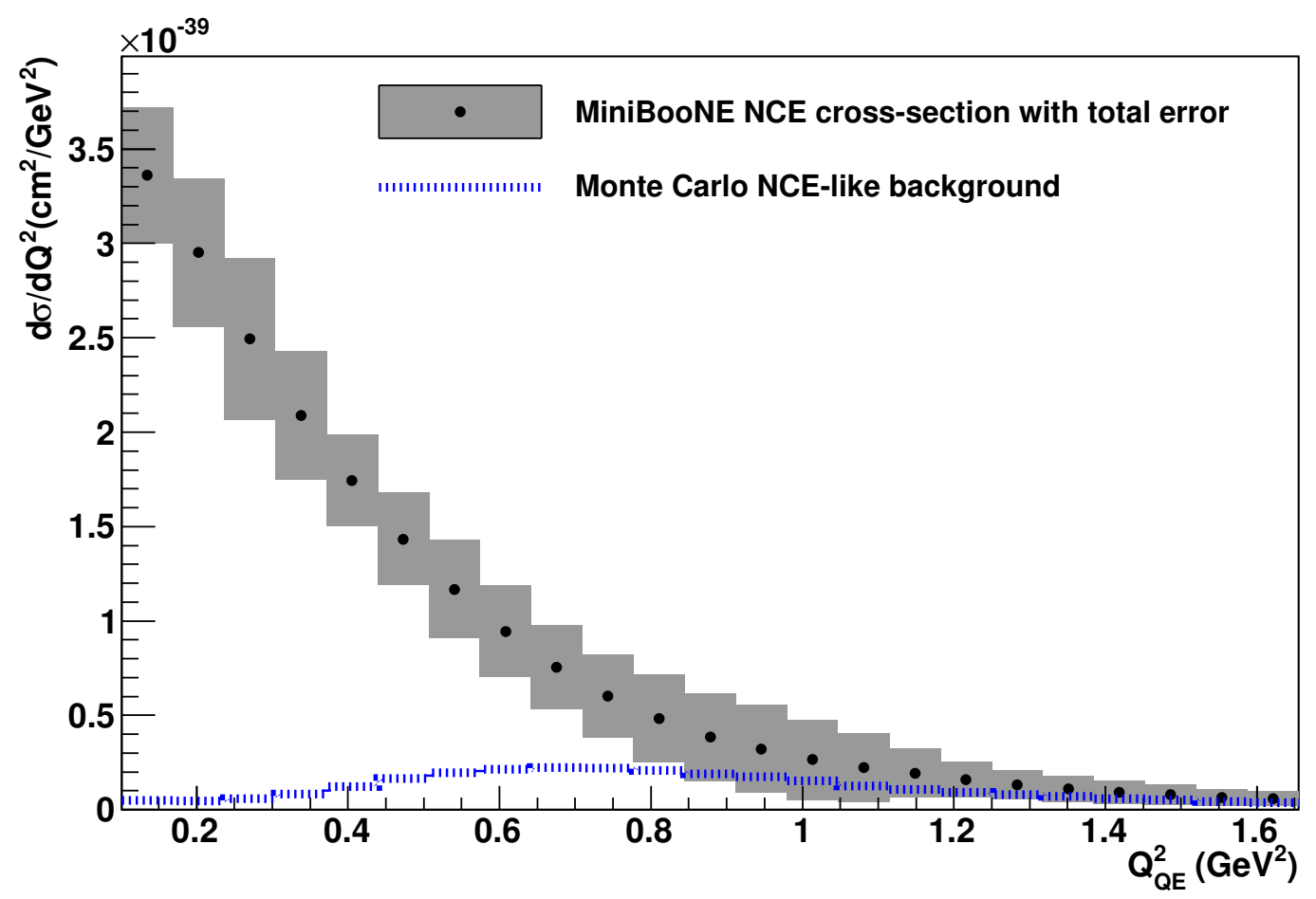

Figure 2.4: The MiniBooNE NCE $(\nu N \rightarrow \nu N)$ flux averaged differential cross section on $\mathrm{CH}_{2}$ as a function of $Q_{Q E}^{2}=2 M_{N} \sum_{i} T_{i}$ where we sum the true kinetic energies of all final state nucleons produced in the NCE interaction. The blue dotted line is the predicted spectrum of NCE-like background which has been subtracted out from the total NCE-like differential cross section [60].

A $\chi^{2}$ goodness of fit test was performed using the reconstructed NCE energy spectrum to find the set of $M_{A}$ and $\kappa$ (Pauli blocking scaling parameter) that best matches the data. Assuming $\Delta s=0$, the MiniBooNE NCE sample yields:

$$
M_{A}=1.39 \pm 0.11 \mathrm{GeV}
$$

with $\chi_{\min }^{2} / D O F=26.9 / 50$.

Even though the ratio $\nu p \rightarrow \nu p$ to $\nu n \rightarrow \nu n$ is more sensitive to $\Delta s$ [63] than the ratio of $\nu p \rightarrow \nu p$ to $\nu N \rightarrow \nu N$ (where $N$ is any nucleon, either $p$ or $n$ ), a neutron can only be detected in MiniBooNE if it has a further strong interaction with a proton, which at low energies is difficult to distinguish from single proton events. Hence a sample of single protons above $350 \mathrm{MeV}$ (the Cherenkov threshold for protons in MiniBooNE) was used and the ratio 
of $\nu p \rightarrow \nu p$ to $\nu N \rightarrow \nu N$ as a function of reconstructed nucleon energy from $350 \mathrm{MeV}$ to $800 \mathrm{MeV}$ was studied to measure $\Delta s$. Additionally looking at such a ratio reduces the effect of FSIs and also some systematic errors. Assuming a value of $M_{A}=1.39 \pm 0.11 \mathrm{GeV}$, the $\chi^{2}$ tests of $\Delta s$ to the MiniBooNE measured $\nu p \rightarrow \nu p$ to $\nu N \rightarrow \nu N$ ratio gives:

$$
\Delta s=0.08 \pm 0.26
$$

with $\chi_{\min }^{2} / D O F=34.7 / 29$. This is consistent with the result obtained by the BNL E734 experiment [62]. 


\section{Chapter 3}

\section{The MiniBooNE Experiment}

In this chapter we start with the motivation for designing the experiment. We then describe the detailed setup for generating the neutrino/antineutrino flux. Next we give a description of the detector itself. Finally we describe our prediction for both the neutrino flux and the detector response.

\subsection{Motivation}

The Mini Booster Neutrino Experiment (MiniBooNE) [64] was proposed to verify or dismiss the possible indication for neutrino oscillations reported by the LSND experiment. In 1997 the Liquid Scintillator Neutrino Detector (LSND) experiment at the Los Alamos Meson Physics Facility, reported an excess of $87.9 \pm 22.4$ (stat) \pm 6.0 (syst) $\bar{\nu}_{e}$ events in a $\bar{\nu}_{\mu}$ beam produced by the decay at rest of positive pions [65]. Assuming that this excess is due to $\bar{\nu}_{\mu} \rightarrow \bar{\nu}_{e}$ oscillations, the LSND signal corresponds to neutrino oscillations with $\Delta m^{2} \sim 1 \mathrm{eV}^{2}$, as shown in Fig. 3.1. This in turn suggests the existence of a new type of neutrino, as the solar and atmospheric neutrinos already set two distinct values for the mass squared differences [1], namely

$$
\Delta m_{\text {sol }}^{2}=\Delta m_{21}^{2}=\left(7.58_{-0.26}^{+0.22}\right) \times 10^{-5} \mathrm{eV}^{2}
$$


and

$$
\Delta m_{a t m}^{2}=\Delta m_{31}^{2}=\left(2.35_{-0.09}^{+0.12}\right) \times 10^{-3} \mathrm{eV}^{2}
$$

Therefore, this new neutrino would have to be sterile, and consequently would point to significant new physics beyond the Standard Model.

In order to be sensitive to the same values of $\Delta m^{2}$, MiniBooNE was designed to have a similar average value of $L / E$ as LSND, where $L=32 \mathrm{~m}$ and $E_{\text {avg }} \simeq 45 \mathrm{MeV}$. However, MiniBooNE operates at higher energies and longer baseline, namely $E_{\text {avg }} \simeq 700 \mathrm{MeV}$ and $L=545 \mathrm{~m}$, respectively, which allows for a cross check of the LSND signal, with completely different signal and backgrounds. 


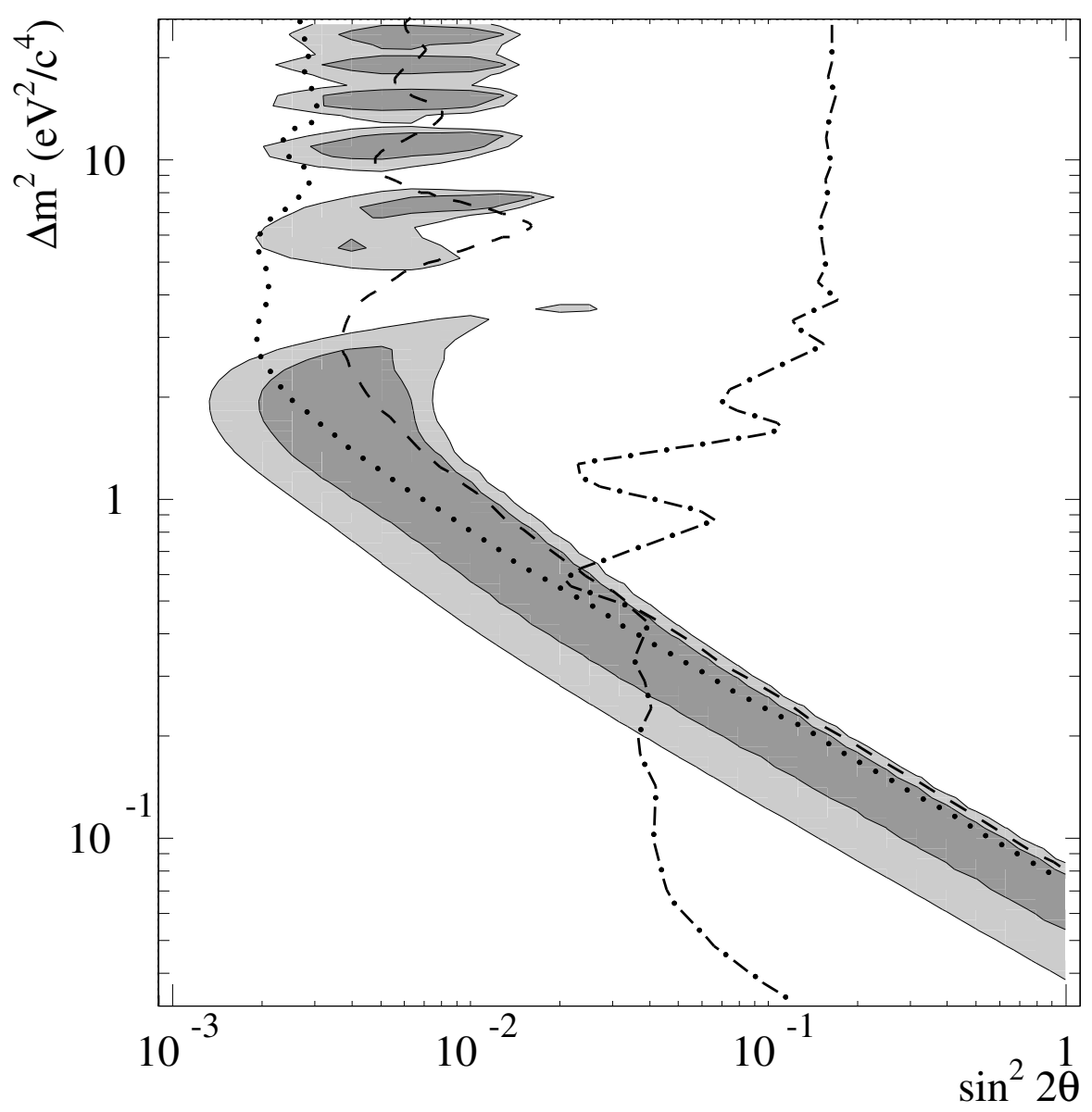

Figure 3.1: The LSND signal. The plot shows the $\left(\sin ^{2} 2 \theta, \Delta m^{2}\right)$ favored regions obtained from the $\bar{\nu}_{\mu} \rightarrow \bar{\nu}_{e}$ decay at rest oscillations search. The dark-shaded region correspond to $90 \%$ likelihood and the light-shaded region correspond to $99 \%$ likelihood. Also shown are the $90 \%$ confidence limits from the KARMEN experiment (dashed), BNL-E776 (dotted), and the Bugey reactor experiment (dot-dashed). Figure taken from Ref. [66].

After 10 years of continuous running, MiniBooNE has not been able to clearly confirm or dismiss the LSND signal. Small excesses have been reported in both the neutrino and antineutrino oscillation channels, but they remain below the $3 \sigma$ level $[67,68,69]$. MiniBooNE has stopped running as of April 24, 2012. Nonetheless, despite the fact that the primary purpose of MiniBooNE was to search for neutrino oscillations, the detector has proven to be very well suited to measure a variety of neutrino cross sections, most of which to yield the best measurements in the world. 


\subsection{The MiniBooNE Experiment}

MiniBooNE is located at the Fermi National Accelerator Laboratory (FNAL, or Fermilab) in Batavia, IL. The experiment began collecting data in 2001 and reported the neutrino mode $\nu_{\mu} \rightarrow \nu_{e}$ oscillation search result in 2007 [67]. Since then it has been running in the antineutrino mode looking for $\bar{\nu}_{\mu} \rightarrow \bar{\nu}_{e}$ oscillations, which is a direct search of the LSND result. Initial antineutrino mode results were published in 2010 [69].

The experimental layout is shown in Fig. 3.2. Protons from the Fermilab Booster are extracted and impinge upon a beryllium target. The resulting mesons decay in flight to neutrinos in the decay region and reach the MiniBooNE detector. A detailed description of the detector hardware can be found in Ref. [70].

\subsection{The MiniBooNE Neutrino Beam}

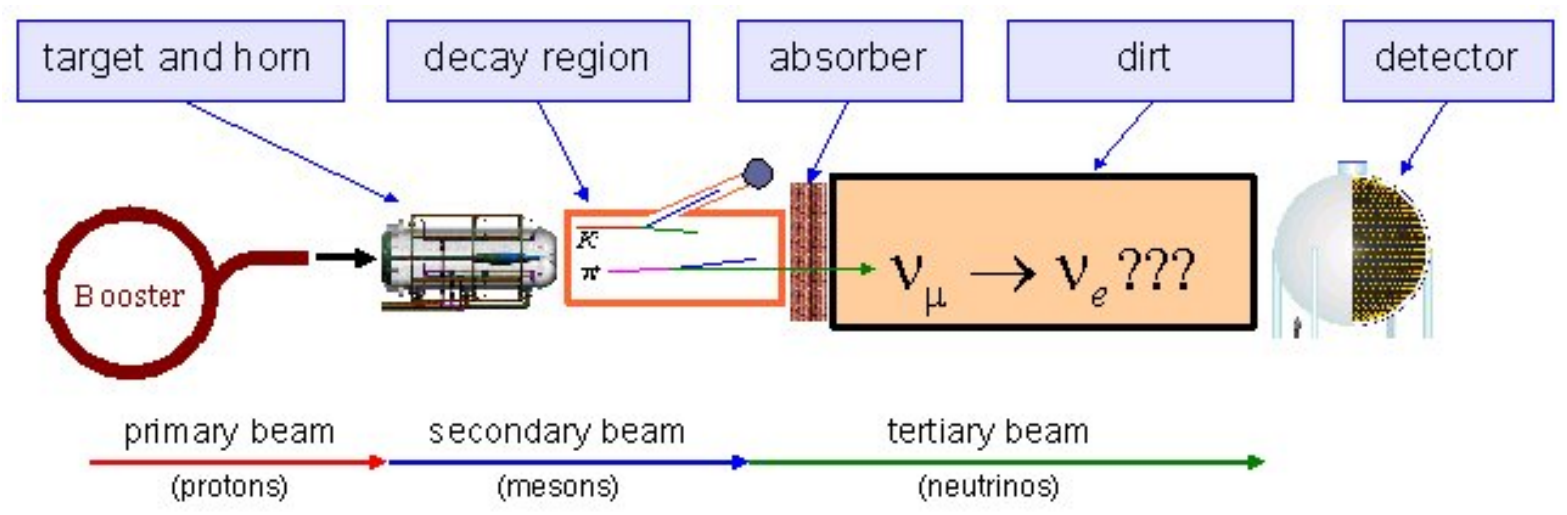

Figure 3.2: Schematic representation of the MiniBooNE experimental setup (not to scale).

Like any other neutrino oscillations experiment, MiniBooNE requires a high neutrino flux and an accurate knowledge of the flux composition at the same time. The MiniBooNE flux production can be divided into 3 stages: the primary proton beam extracted from the Booster, the secondary meson beam which is the result of proton-target interaction, and finally the tertiary neutrino beam which results from the meson decay. The rest of this section describes each of these stages in detail. 


\subsubsection{Primary Proton beam}

The primary protons begin their journey at the Cockroff-Walton generator where hydrogen gas is turned into $\mathrm{H}^{-}$ions and accelerated out by the $750 \mathrm{keV}$ electrostatic gap of the Cockroff-Walton generator. The next step is the linear accelerator (LINAC) which ramps up the $\mathrm{H}^{-}$ions to $400 \mathrm{MeV}$ kinetic energy. Right before entering the next accelerator, the Booster, the $\mathrm{H}^{-}$ions pass through a stripping foil which converts them into $\mathrm{H}^{+}$ions (protons). The Fermilab Booster is a $468 \mathrm{~m}$ circumference synchrotron where the proton beam kinetic energy is boosted to $8.89 \mathrm{GeV}$ and sent towards the Main Injector via a transfer beamline. Beam extraction from the Booster ring is done in a single turn by a kicker magnet.

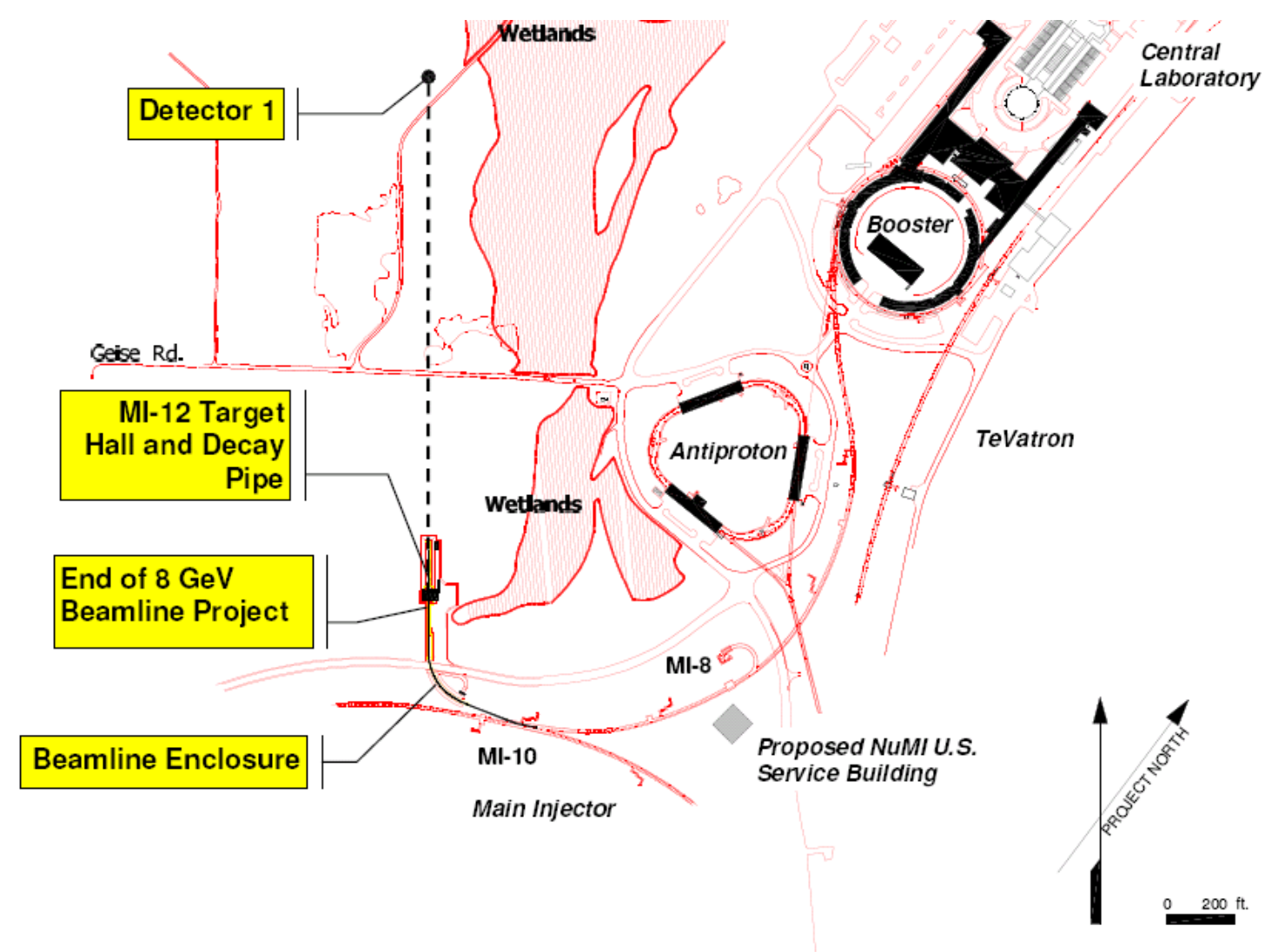

Figure 3.3: The Booster neutrino beamline. Figure appears in Ref. [64]

Each extracted collection of protons is called a spill. Each spill has, on average, $4 \times 10^{12}$ protons. The spills are not uniform in structure; the protons are divided into 81 bunches, each approximately $6 \mathrm{~ns}$ wide and $19 \mathrm{~ns}$ apart. The 81 bunches define the microstructure 
of the beam; they combine to a macrostructure approximately $1.6 \mu$ s wide within a $19.2 \mu \mathrm{s}$ window (as shown in Fig. 3.4). At the end of the transfer beamline, just before the Main Injector, a switch magnet diverts the spills to the Booster Neutrino Beamline (BNB). The BNB contains the MiniBooNE target, onto which the beam is focused using a series of dipole and focusing-defocusing magnets.

The beam position and width is known to within $0.1 \mathrm{~mm}$ due to the beam position monitor (BPM) and a multiwire chamber. The beam current is measured by two toroids upstream of the target. Together they can measure the number of protons on target (POT) to within $2 \%$.

(a)

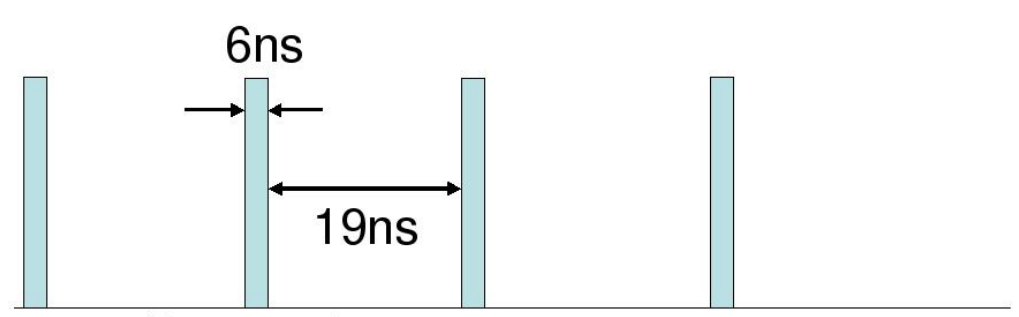

Beam micro structure

(b)

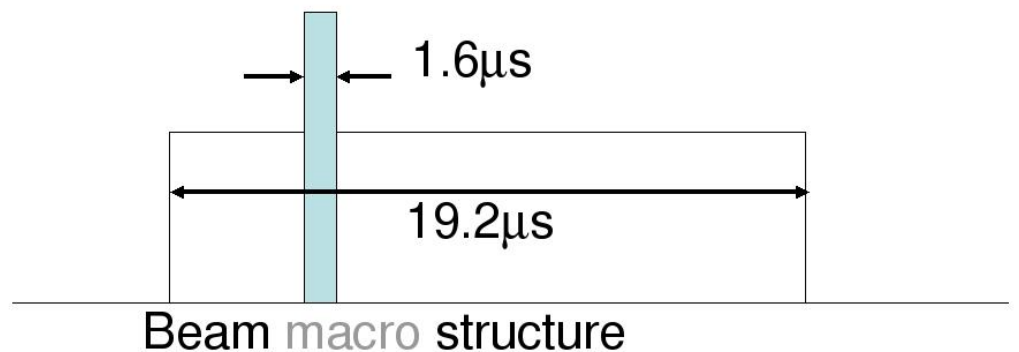

Figure 3.4: The microstructure (a) and macrostructure (b) of the proton beam.

\subsubsection{Secondary meson beam}

The MiniBooNE target consists of seven cylindrical beryllium slugs, which add up to $71.12 \mathrm{~cm}$ in total length. The slugs are enclosed in two beryllium tubes enclosed by a beryllium cap. The protons impinging on the beryllium target create a shower of secondary particles, 
mostly pions $\left(\pi^{+}\right.$and $\left.\pi^{-}\right)$and kaons $\left(\mathrm{K}^{+}, \mathrm{K}^{+}\right.$, and $\left.\mathrm{K}^{0}\right)$. The proton target interaction generates heat which necessitates continuous cooling of the target. The target is air cooled by circulating air between the slugs via tubes which open into the target assembly.

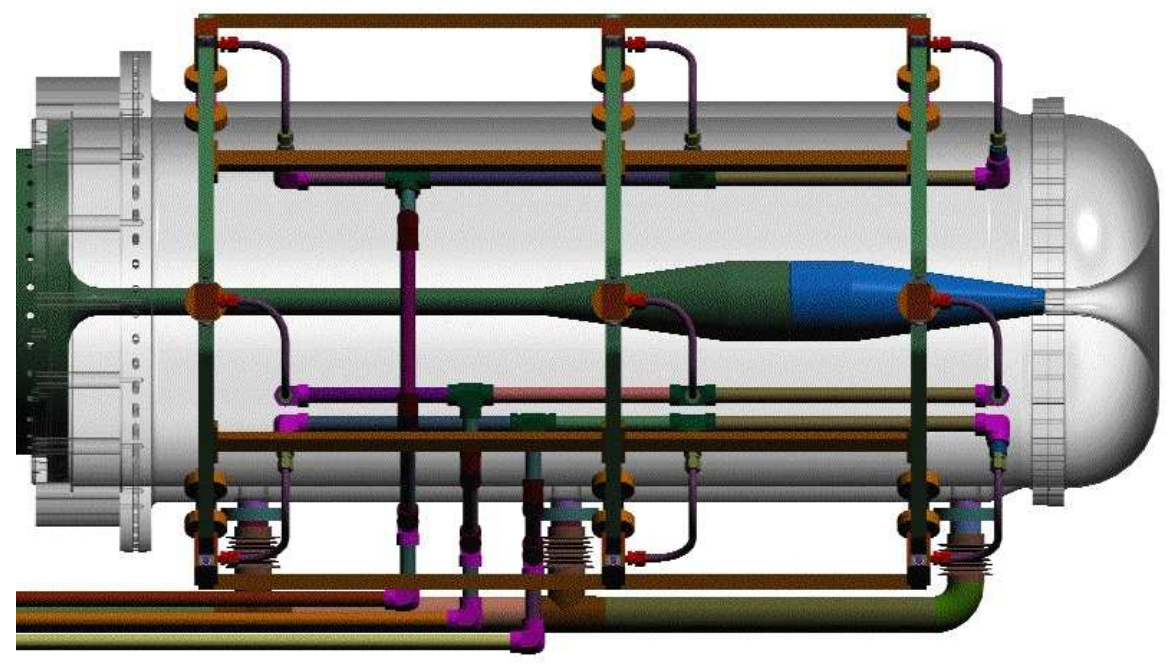

Figure 3.5: The MiniBooNE magnetic focussing horn. Also seen are the plumbing for water cooling. Figure from Ref. [71], courtesy of Bartoszek Engineering [72]

The whole target assembly is placed in a pulsed electromagnet called the "horn", as shown in Fig. 3.5. The horn is a toroidal electromagnet which sign selects the positive or negative mesons depending on the direction of current flow. The magnetic field additionally increases the neutrino flux at the detector by a factor of 6 . The horn operates on a $173 \mathrm{kA}$ peak current resulting in a peak magnetic field of $1.5 \mathrm{~T}$. The horn also has additional plumbing to water cool itself. A collimator located downstream from the horn removes large angle particles before the next stage.

\subsubsection{Tertiary neutrino beam}

After passing through the collimator the secondary meson beam enter the decay pipe. It is a $2 \mathrm{~m}$ wide and $50 \mathrm{~m}$ long air-filled decay region where the mesons decay in flight (DIF) to 
neutrinos. The primary source of neutrino is the decay of pions and kaons. At the end of the decay pipe there is a steel and concrete beam stop to stop all particles except neutrinos to pass through. After exiting the decay region the neutrinos travel through about $540 \mathrm{~m}$ of dirt. This is the oscillation region where the neutrino oscillation can take place before the beam reaches the detector.

\subsection{The MiniBooNE Neutrino Flux}

MiniBooNE employs the GEANT4 simulation package [73] to model the neutrino flux. GEANT (GEometry ANd Tracking) uses Monte Carlo methods to describe the passage of elementary particles through matter. The framework can account for energy losses and electromagnetic and hadronic processes that alter the kinematics of the propagating particles. Within each medium, Coulomb scattering and energy loss are calculated in each step of the tracking and particle trajectory updated accordingly. GEANT4 is also capable of tracking charged particles in a magnetic field.

GEANT4 accepts as input the full geometry of the domain to be simulated, as well as the initial conditions of the particles.

The GEANT4 geometry consists of the last $50 \mathrm{~m}$ of the booster beamline, the MiniBooNE target hall, and the meson decay volume. It specifies the shape, location and composition of the components of BNB through which the the primary protons and other particles propagate. The geometry description is defined to match the actual beamline as closely as possible, with simplifications made which are not expected to affect the flux predictions.

The initial conditions of particles used for GEANT4 input depends on the specific process in the beamline and comes from a combination of sources which includes other particle production software, external measurements by MiniBooNE or other experiments in similar energy regime, theoretical predictions, and sometimes extrapolation of measurements to MiniBooNE energies. The details for each of the processes are discussed below. 


\subsubsection{Modelling the Primary Proton Beam and Horn}

The beam optics characteristics are used to generate the primary protons before the target and the TRANSPORT software package [74] was used to simulate properties like position and profile. The rate of interactions for protons, neutrons, and charged pions on beryllium and aluminum are determined by customized cross section tables. The cross sections for these particles fall into three categories: elastic scattering, inelastic scattering, and quasielastic scattering. The Glauber model [75] was used to calculate the total cross section and subtracting the measured inelastic cross section to give the elastic cross section. In the case of inelastic scattering, available measurements for $\mathrm{p}-\mathrm{Be}$ and $\mathrm{p}-\mathrm{Al}$, in the momentum range of MiniBooNE exist [76]. For quasi-elastic scattering, very few measurements are available, hence we make use of theoretical calculations. The calculation along with the sparse measurements are extrapolated for beryllium. For explicit forms of the cross sections which are inspired by the Regge theory [77], see Ref. [78].

\subsubsection{Secondary Particle Production Model}

Of special interest to MiniBooNE is the p-Be interaction at $8.89 \mathrm{GeV}$. Most of the neutrinos seen by MiniBooNE come from the decay of $\pi^{+}$and $\pi^{-}$and other associated secondaries of the p-Be interaction at this particular energy. The HARP experiment [79] at CERN studied the pion production on a replica Be target at MiniBooNE energy. The HARP measurements were used to generate the $\pi^{ \pm}$production tables and a Sanford and Wang model [80] was used to parametrize the differential production cross section at different incident primary beam momenta and extrapolate it to regions where production data do not exist.

The Sanford-Wang parametrization is given by

$$
\frac{d^{2} \sigma}{d p d \Omega}=c_{1} p^{c_{2}}\left(1-\frac{p}{p_{B}-c_{9}}\right) \exp \left[-c_{3} \frac{p^{c_{4}}}{p_{B}^{c_{5}}}-c_{6} \theta\left(p-c_{7} p_{B} \cos ^{c_{8}} \theta\right)\right]
$$

where $p_{B}$ is the incident proton beam momentum, $p$ is the outgoing meson momentum, and 
$\theta$ is the outgoing meson direction relative to the proton direction. Fits to the available measurements of the differential cross section $d^{2} \sigma / d p d \Omega$ were done using a $\chi^{2}$ minimization to determine the $c_{1}-c_{9}$ Sanford-Wang parameters for each meson. Figure 3.6 shows data from the HARP experiment fit to the Sanford-Wang parametrization above.

Data from the BNL E910 experiment [81] which measured $\pi^{ \pm}$differential cross section for $\mathrm{p}$-Be interaction at different energies was used to cover the energy regime not covered by the HARP data.

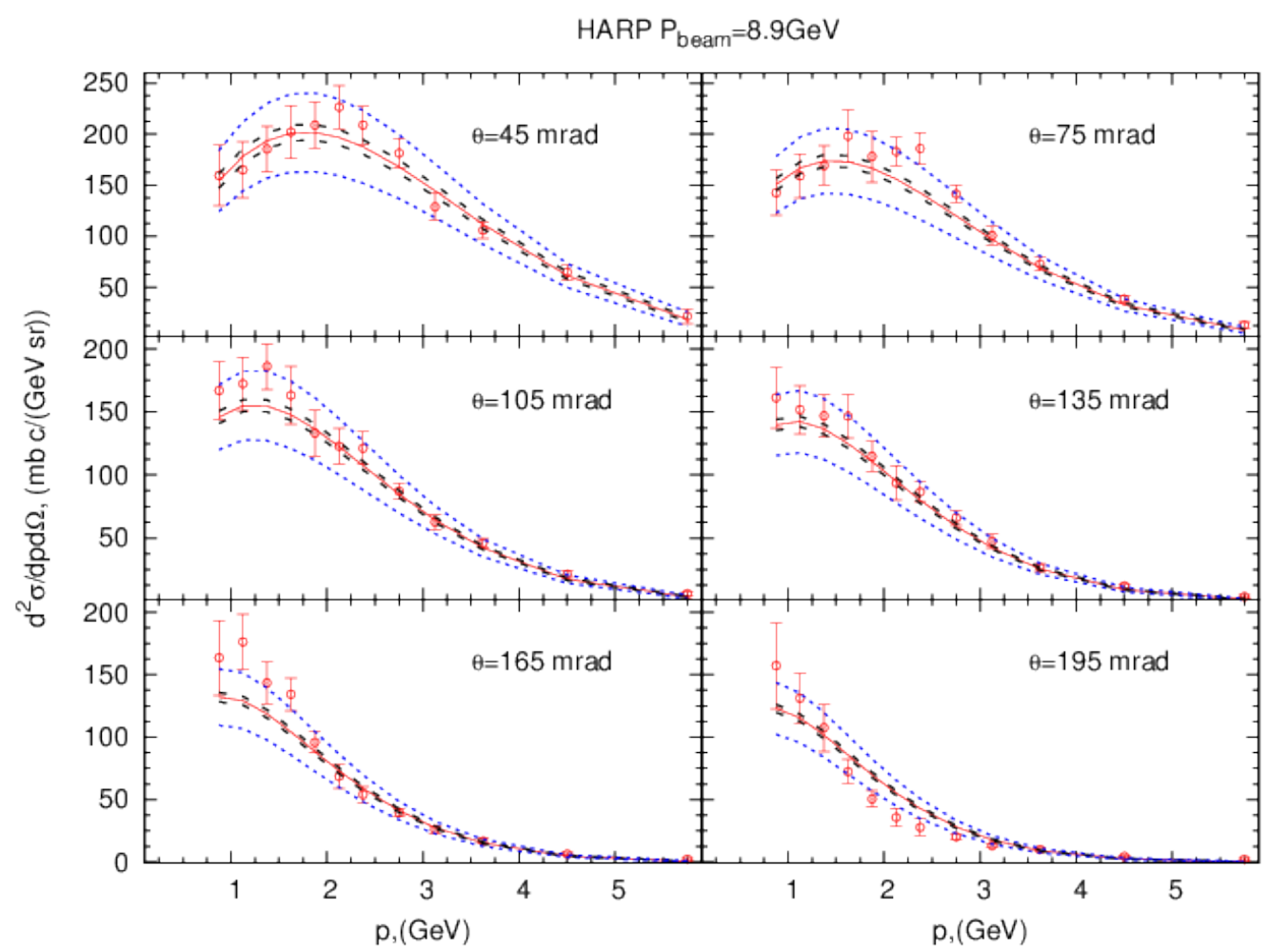

Figure 3.6: The Sanford-Wang curves for $\pi^{+}$production (red curve) with HARP data points (red points). The blue bands are the uncertainties assigned. Figure taken from Ref. [78]

For $\mathrm{K}^{+}$production, since no measurements at $8.89 \mathrm{GeV}$ exist, measurements by other experiments $[82,83]$ close to $8.89 \mathrm{GeV}$ are used and the Feynman scaling hypothesis is employed to extrapolate to MiniBooNE energies. The $\mathrm{K}^{0}$ 's have an equal content of $K_{L}^{0}$ 's and $K_{S}^{0}$ 's. The long lifetime of $K_{L}^{0}$ 's reduces the neutrino contribution and the $K_{S}^{0}$ 's production properties can be measured from the external measurements [84]. Once again, a Sanford- 
Wang parametrization of these data was employed to extrapolate to MiniBooNE energies and constrain the neutral kaons.

Neutrino flux contributions from $\mathrm{K}^{+}$and other semileptonic hyperon decays (e.g., $\Lambda, \Sigma$ ) are negligible. In the absence of measured data, the MARS hadronic interaction package [85] was used to estimate the $\mathrm{K}^{+}$production. The FLUKA simulation package [86] was used to constrain the semileptonic hyperon decays.

The results of the neutrino flux simulations are shown in Fig. 3.7 for both the neutrino and antineutrino running modes. 

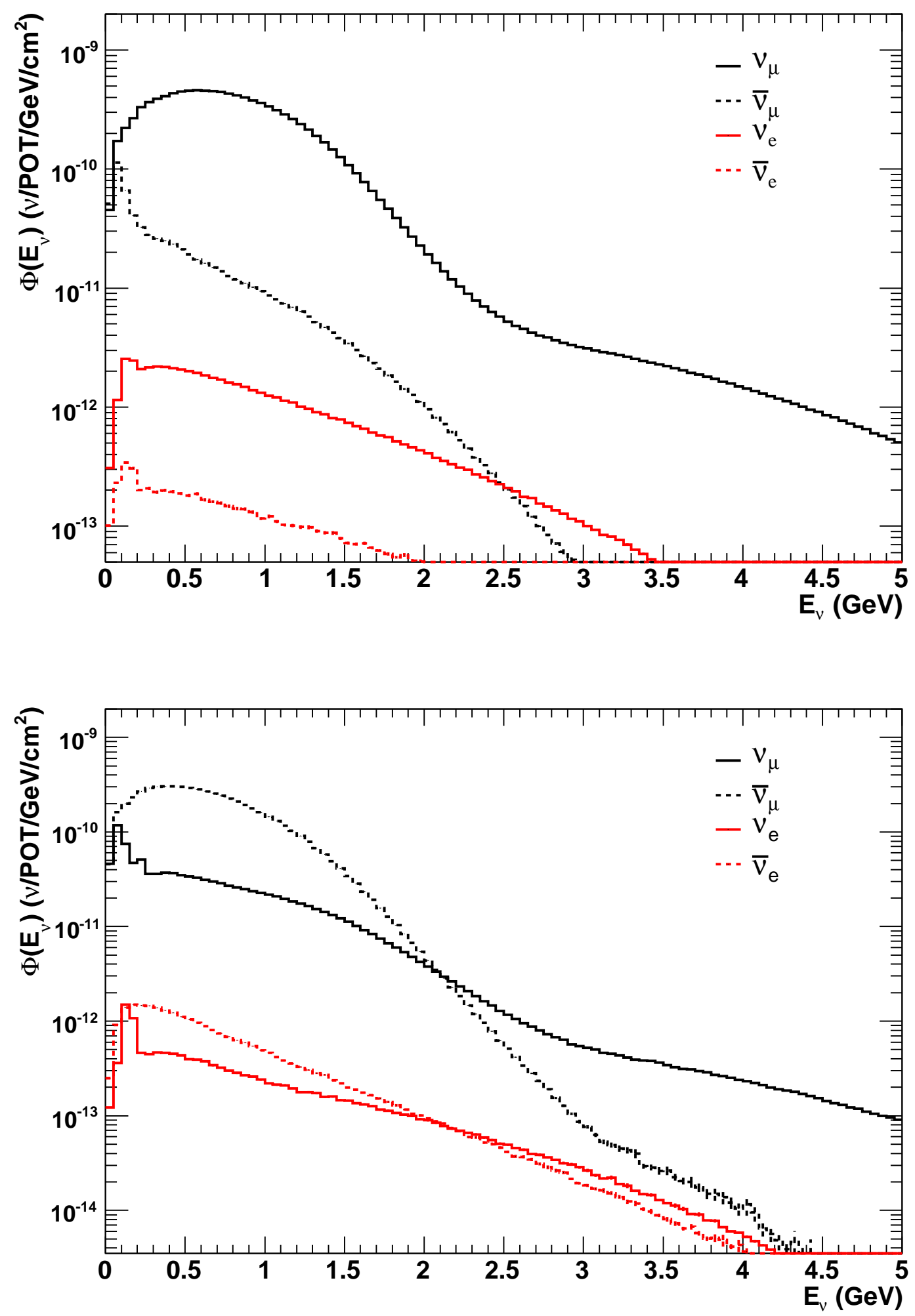

Figure 3.7: The MiniBooNE flux predictions for the neutrino and antineutrino modes, top and bottom, respectively. The solid lines show the $\nu_{\mu}$ (black) and $\nu_{e}$ (red) while the dashed lines show the $\bar{\nu}_{\mu}$ (dashed black) and $\bar{\nu}_{e}$ (dashed red). Note the neutrino background in the antineutrino mode is higher than the corresponding antineutrino background in the neutrino mode.Figure taken from Ref. [78] 


\subsection{The MiniBooNE Detector}

The MiniBooNE detector is a mineral oil Cherenkov detector, as shown schematically in

Fig. 3.8. It is a $610 \mathrm{~cm}$ radius sphere with two optically isolated regions separated by a spherical shell of radius $575 \mathrm{~cm}$. The inner region, also referred to as the signal region is lined with 1,280 8-inch photomultiplier tubes (PMTs) pointed inwards. The outer region, which is the veto region, has 240 PMTs arranged in back-to-back pairs pointed along the circumference of the detector. The signal region is painted black to minimize reflections, whereas the veto region is painted white in order to maximize light collection at the PMTs.

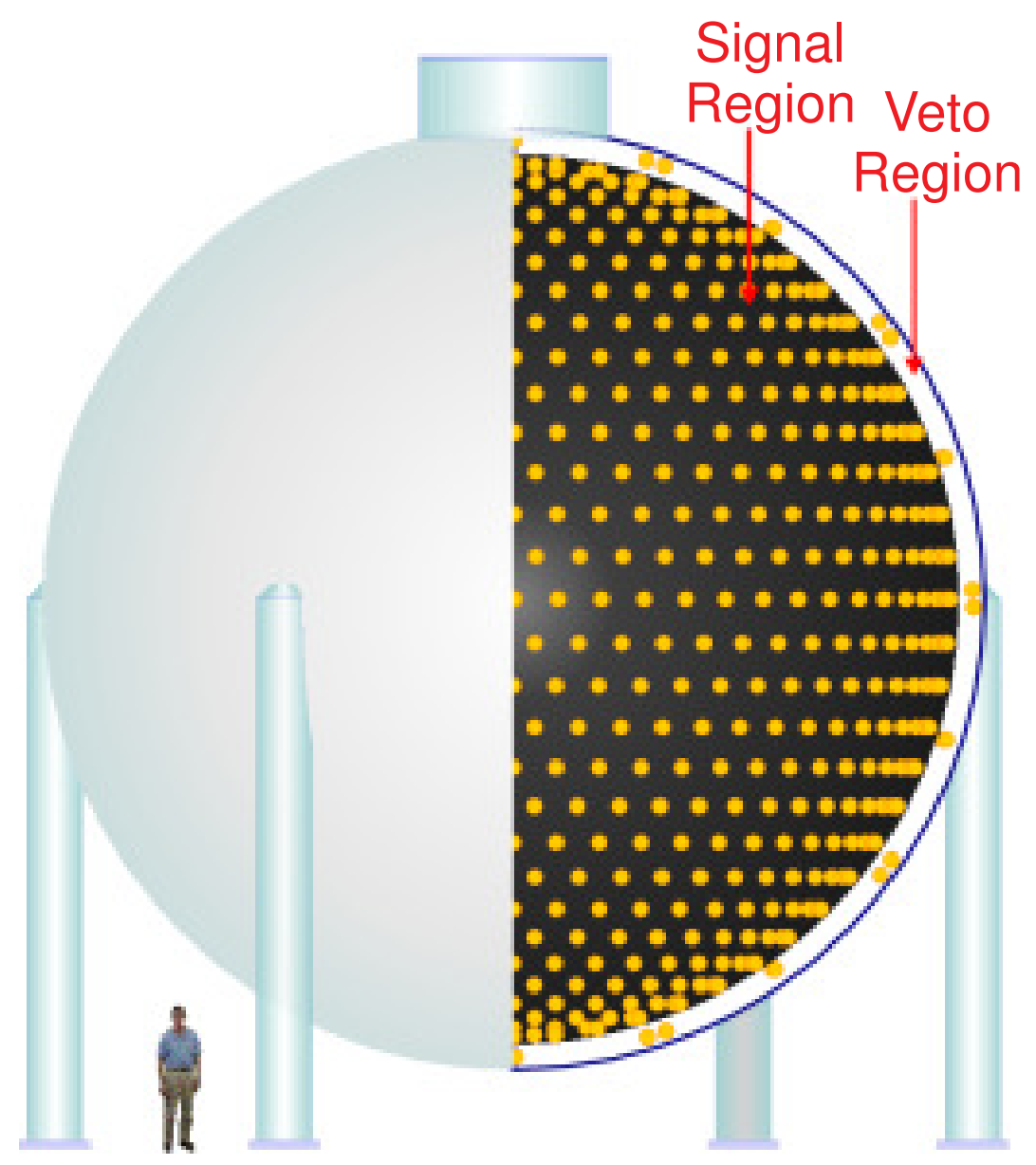

Figure 3.8: A schematic of the MiniBooNE detector. From Ref. [87].

Figure 3.9 shows a snapshot of the signal and veto regions of the detector, which also illustrates the PMT orientations in the two volumes. 


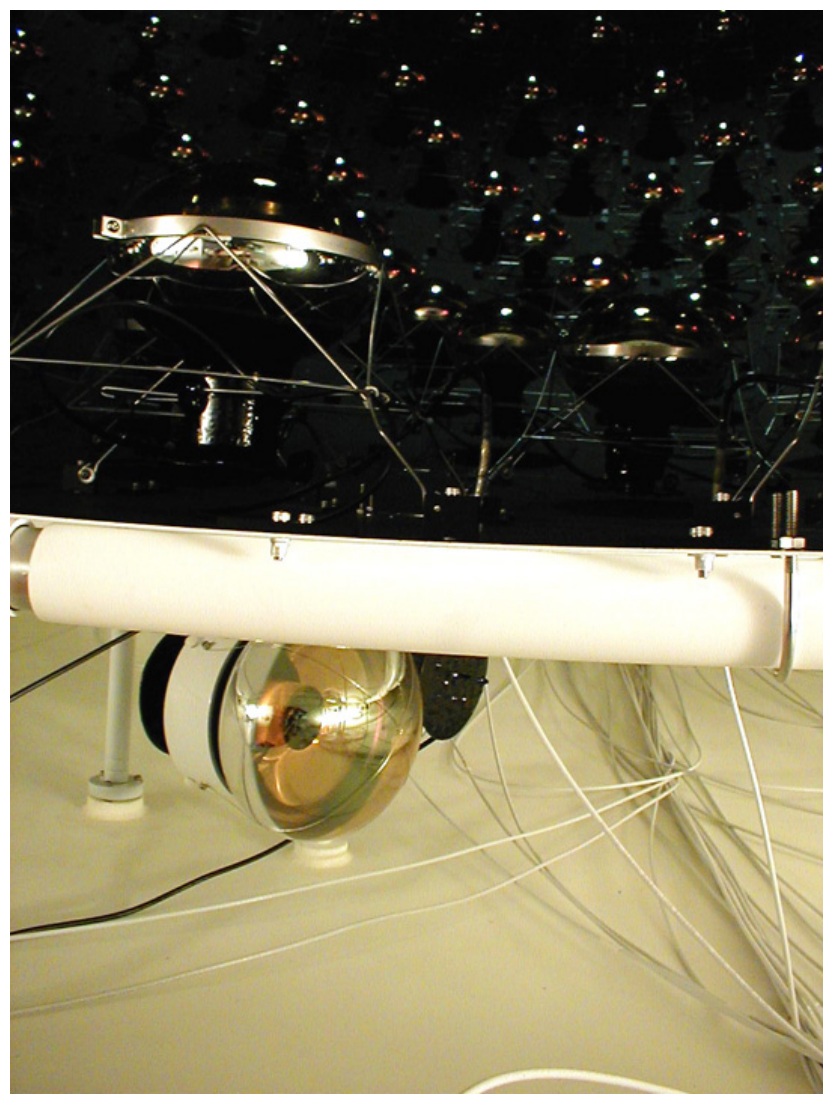

Figure 3.9: A snapshot of the signal and veto regions. Note the orientations of the PMTs in the two regions and the colors of the two regions - the signal region is painted black to minimize reflections, whereas the veto region is painted white to maximize light collection.

The detector is supported by six steel legs and is situated in a vault at about $545 \mathrm{~m}$ from the MiniBooNE target. The area above the vault houses the detector electronics and DAQ systems, as well as the pumps for oil circulation. The entire assembly is buried under approximately $3 \mathrm{~m}$ earth equivalent of overburden - see Fig. 3.10. This overburden reduces somewhat the number of cosmic muons entering the detector (however, the main reduction of the cosmic-ray backgrounds comes from the shortness of the beam spills). Further details of the MiniBooNE detector can be found in Ref. [70]. 


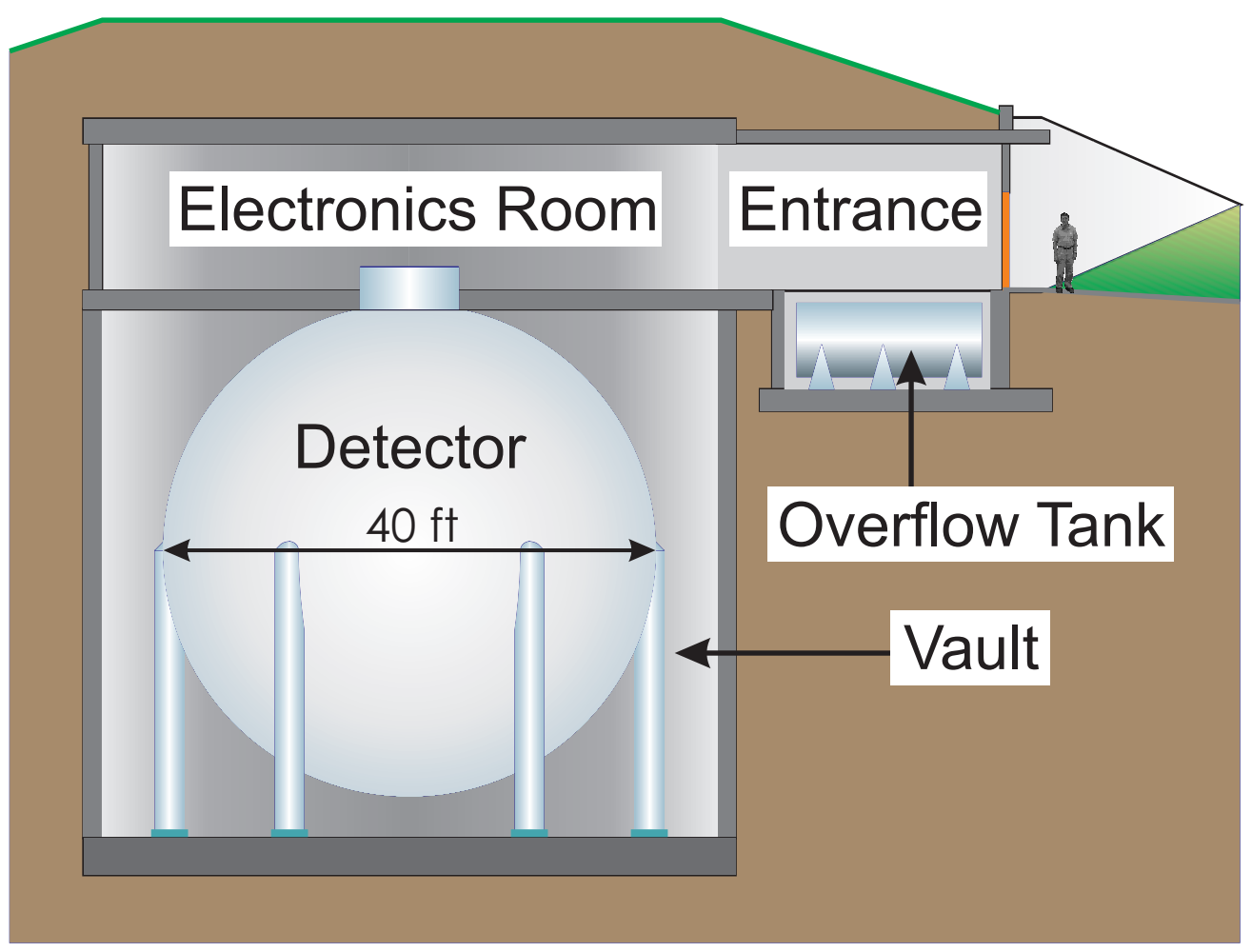

Figure 3.10: A schematic of the MiniBooNE detector in the vault. Also shown is the electronics room at the surface level, the location of the overflow tank, and the overburden. From Ref. [87].

\subsubsection{The MiniBooNE Target-Mineral oil}

The detector is filled with 807 tons of Marcol-7 (produced by EXXON/MOBIL) mineral oil which functions as the target for the neutrino beam as well as the medium for light production. The choice of this particular detector medium was driven by various physics goals of MiniBooNE.

Mineral oil has a higher index of refraction $(n=1.47)$ as compared to water $(n=1.33)$ which improves the sensitivity to lower energy particles like protons and corresponds to higher Cherenkov yields. Having an index of refraction closely matching that of the PMT glass $(n \simeq 1.5)$ also minimizes the losses through reflections. A lower Cherenkov threshold for heavy particles like protons allows to measure their direction. Mineral oil also has low $\mu^{-}$capture rate as compared to water. This improves our ability to identify charged current neutrino interactions as we rely on the Michel electron from the $\mu^{-}$decay for tagging such 
events. The light extinction length of the MiniBooNE oil is approximately $18 \mathrm{~m}$.

Detailed studies of the various properties of the oil were carried out. As an example, the temperature and wavelength dependence of the refractive index were measured using an Abbe refractometer, to yield [88]

$$
n(\lambda, T)=\left[n_{D}+B\left(\frac{1}{\lambda^{2}-\lambda_{D}^{2}}\right)\right]\left[1-\beta\left(T-T_{0}\right)\right]
$$

where the index of refraction was measured at the mean of the Fraunhofer $D_{1}$ and $D_{2}$ lines - the sodium doublet $\lambda_{D}=589.3 \mathrm{~nm}$. Here $n_{D}=1.4684 \pm 0.0002, B=42040 \pm 157 \mathrm{~nm}^{2}$, $\beta=(3.66 \pm 0.04) \times 10^{-4} /{ }^{\circ} \mathrm{C}$, and $T_{0}=20^{\circ} \mathrm{C}$. Details of all studies performed on the MiniBooNE mineral oil can be found in Ref. [89].

Nitrogen gas is continuously bubbled through the oil so as to purge it of oxygen, which is corrosive for the detector structure, and a nitrogen environment is maintained within the detector. Mineral oil needs no continuous purification like water; nothing grows in it and once it is clean, any remaining impurities will either float to the surface or sink to the bottom of the detector. An overflow tank is provided to cope with any thermal expansion of the oil.

\subsubsection{The Photomultiplier Tubes}

A total of 1,520 PMTs are used in the MiniBooNE detector. Of these, 1,198 are Hamamatsu R1408 recycled from the LSND experiment, and the remaining 322 are Hamamatsu R5912 purchased specifically for MiniBooNE. Figure 3.11 shows an inner region PMT with the supporting steel wire structure. 


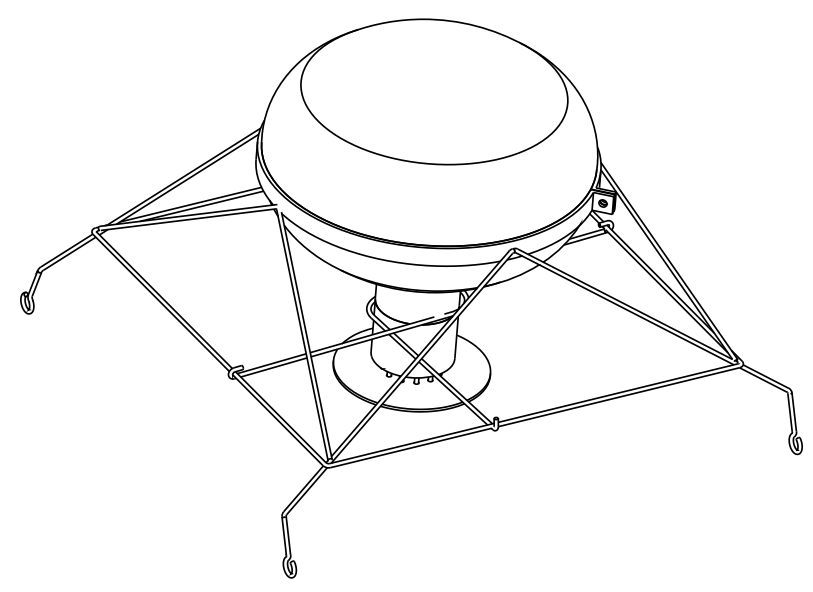

Figure 3.11: A schematic of a signal region PMT with the support wires which are used to attach it to the spherical shell separating the signal region from the veto region. From Ref. [87].

Both the R1480 and the R5912 PMTs, referred to as old and new PMTs, respectively, are of 8 inch in diameter and have similar geometry and quantum efficiency; the new PMTs however have a slightly higher light collection efficiency. Since the new PMTs have a better time resolution $\sim 1.1 \mathrm{~ns}$ and a better single photoelectron (SPE) charge resolution (about $50 \%$ ) as compared to the old ones (with a time resolution of $1.7 \mathrm{~ns}$ and a SPE charge resolution of about 140\%), all the new PMTS are equally dispersed in the signal region. In the veto region 240 of the old PMTS with the lowest dark rate are used. Figure 3.13 shows the layout of old and new PMTs in the detector.

All the PMTs were tested at Fermilab in a dark room to study their time and charge resolution, dark rate, angular acceptance and operating voltage. Details on the PMT design and testing can be found in Refs. [90, 91]. 


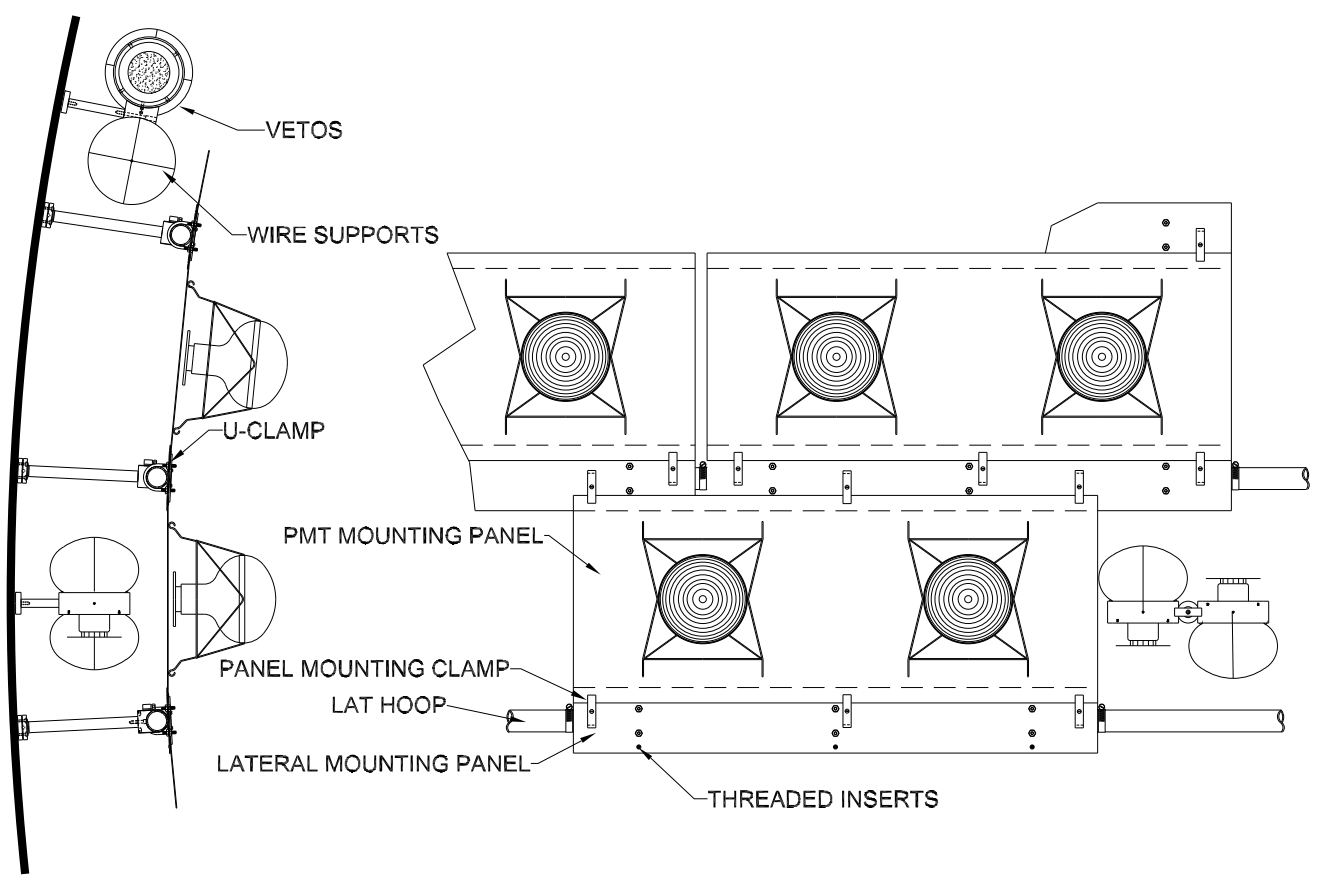

Figure 3.12: The MiniBooNE PMT support structure. Also shown is the location and orientation of the veto PMTs. From Ref. [87].

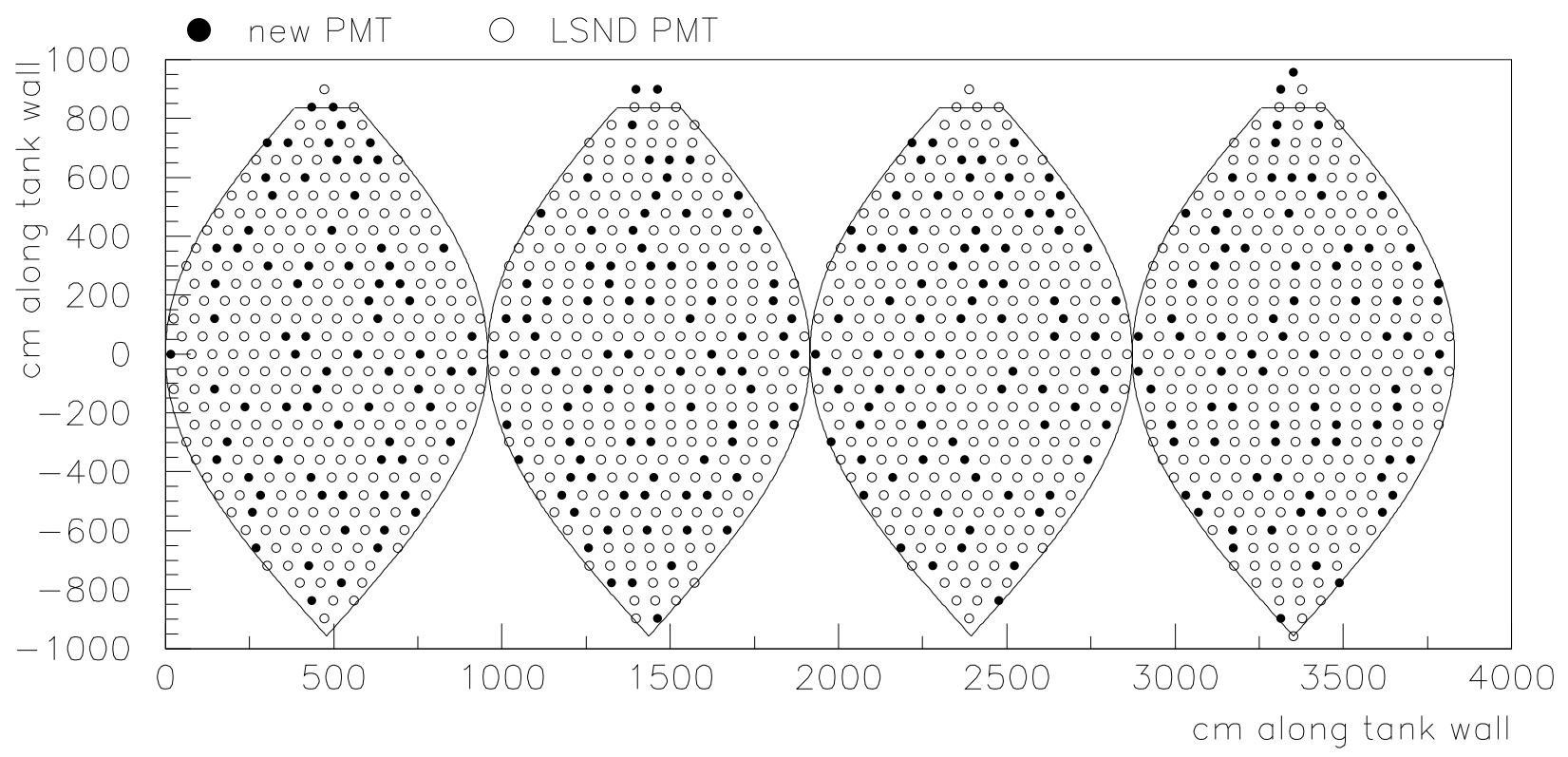

Figure 3.13: Distribution of the old and new PMTs within the MiniBooNE detector, as labeled. From Ref. [87]. 


\subsection{Data Acquisition, Digitization and Trigger}

In the MiniBooNE data acquisition (DAQ), the digitization of signals and data storage is achieved in conjunction with a trigger system, which is a set of conditions which, when true, stores the corresponding information in the DAQ system as data. We describe below how the data acquisition and digitization is achieved followed by a brief description of the trigger system.

\subsubsection{Data Acquisition and Digitization}

The MiniBooNE data acquisition and associated systems are housed in the detector hall above the vault where the detector is situated. High voltage (HV) to the PMTs and the signals from them to be digitized are carried by equal length cables. The overall charge and time associated with each PMT hit is recorded.

A schematic of the charge and time information is shown in Fig. 3.14. The system is driven by a $10 \mathrm{MHz}$ clock. The preamplified PMT signal $V_{p m t}$ is continuously integrated via a capacitive circuit in the so-called QT boards, with an exponential decay time of about $1200 \mathrm{~ns}$, generating a second signal $V_{q}$. If the PMT signal $V_{p m t}$ exceeds 0.25 photoelectron equivalent, a PMT discriminator signal is activated, and a linear time ramp $V_{t}$ is started. The time signal $V_{t}$ is also continuously digitized every $100 \mathrm{~ns}$. The time ramp is reset to baseline when two 100 ns time intervals are elapsed after the PMT signal crossed threshold. The linear extrapolation of the $V_{t}$ and $V_{t+1}$ values to the baseline allows to determine the so-called "fine time", i.e., the hit time.

The charge and time ADC (Analog-to-Digital Converter) values are stored in $204.8 \mu \mathrm{s}$ circular dual port buffers. If the trigger system (explained below) responds to the stored charge and time, the values are passed on to storage, otherwise they are overwritten. For each PMT hit four consecutive values of $V_{q}$ and $V_{t}$ (each of them therefore called a quad) are stored in the buffer. These quads are later used to calculate the charge and time of the 
hit PMT's, if the event is read out.

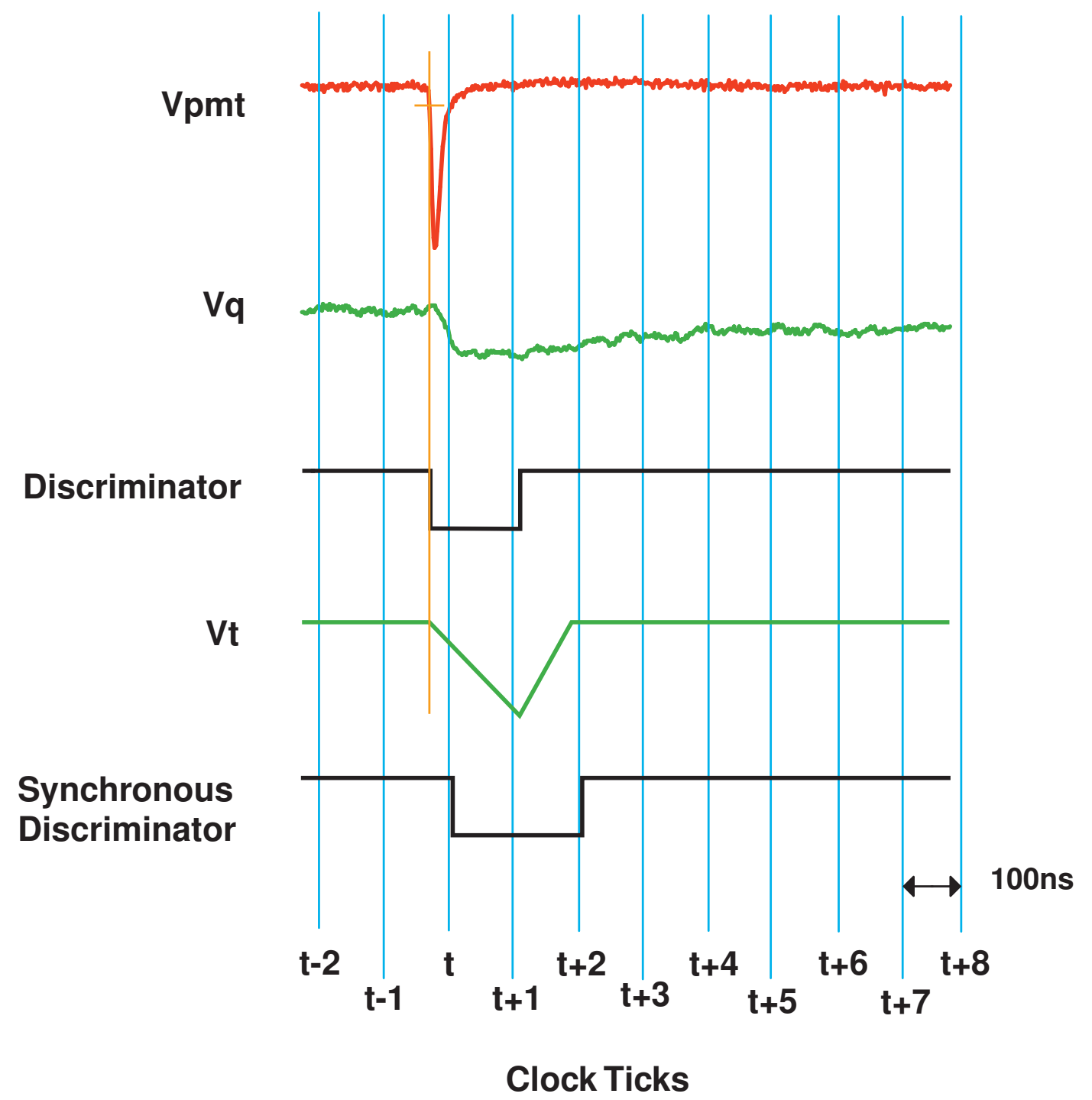

Figure 3.14: A schematic diagram of MiniBooNE signal processing. The schematic appears in Ref. [87]

\subsubsection{The Trigger System}

The MiniBooNE trigger system is a set of conditions which, when true, instructs the DAQ system to store the information currently in the buffer. The trigger system collects information from 3 external triggers, as well as seven comparators in a $200 \mathrm{~ns}$ window. The external sources are two from the accelerator and one from the calibration systems; the comparator levels correspond simply to seven different levels of PMT hit multiplicities. Details of 
MiniBooNE external triggers and comparator settings are as listed in Table 3.1.

\begin{tabular}{lll} 
Input & PMT hits & Purpose \\
\hline \hline External 1 & $\mathrm{n} / \mathrm{a}$ & Beam to MiniBooNE \\
External 2 & $\mathrm{n} / \mathrm{a}$ & Strobe, NuMI, Debuncher \\
External 3 & $\mathrm{n} / \mathrm{a}$ & Calibration Event \\
\hline Comparator 1 & Tank hits $\geq 10$ & Activity Monitor \\
Comparator 2 & Tank hits $\geq 24$ & Michel electron \\
Comparator 3 & Tank hits $\geq 200$ & High-Energy Neutrino \\
Comparator 4 & Tank hits $\geq 100$ & $\nu$ candidate \\
Comparator 5 & Tank hits $\geq 60$ & Supernova $\nu$ candidate \\
Comparator 6 & Tank hits $\geq 6$ & Cosmic Veto \\
Comparator 6 & Tank hits $\geq 4$ & Cosmic Activity \\
\hline
\end{tabular}

Table 3.1: MiniBooNE trigger inputs and comparator settings. From Ref. [87]

The principal physics trigger requirement is that beam was sent to the MiniBooNE target, regardless of the activity in the detector. When this trigger is set, any activity in the detector before and after the beam spill is recorded. When the beam trigger is set, $19.2 \mu \mathrm{s}$ of data are read beginning $5 \mu$ s before the beam arrives. There are also a number of special purpose triggers for calibration (such as the Michel electron, laser, or strobe trigger) or to record various physics event samples (such as the NuMI or supernova trigger). The detector takes data for a $19.2 \mu \mathrm{s}$ window of which $1.6 \mu \mathrm{s}$ is the beam time. The beam-off data recorded during the remaining $17.6 \mu \mathrm{s}$ is used to remove the cosmic ray event contamination - see Fig. 3.4(b). Additionally, there is also a cosmic trigger (Comparator 6 in Table. 3.1) which is used to ignore cosmic ray contaminated data during beam up-time. 


\subsection{Calibration}

MiniBooNE employs two in situ calibration tools: a pulsed laser calibration system and a cosmic muon calibration system. In addition, calibration is also done by using MiniBooNE data to reconstruct certain known physical quantities, such as the $\pi^{0}$ mass and the Michel electron energy spectrum. This section describes these methods in some detail.

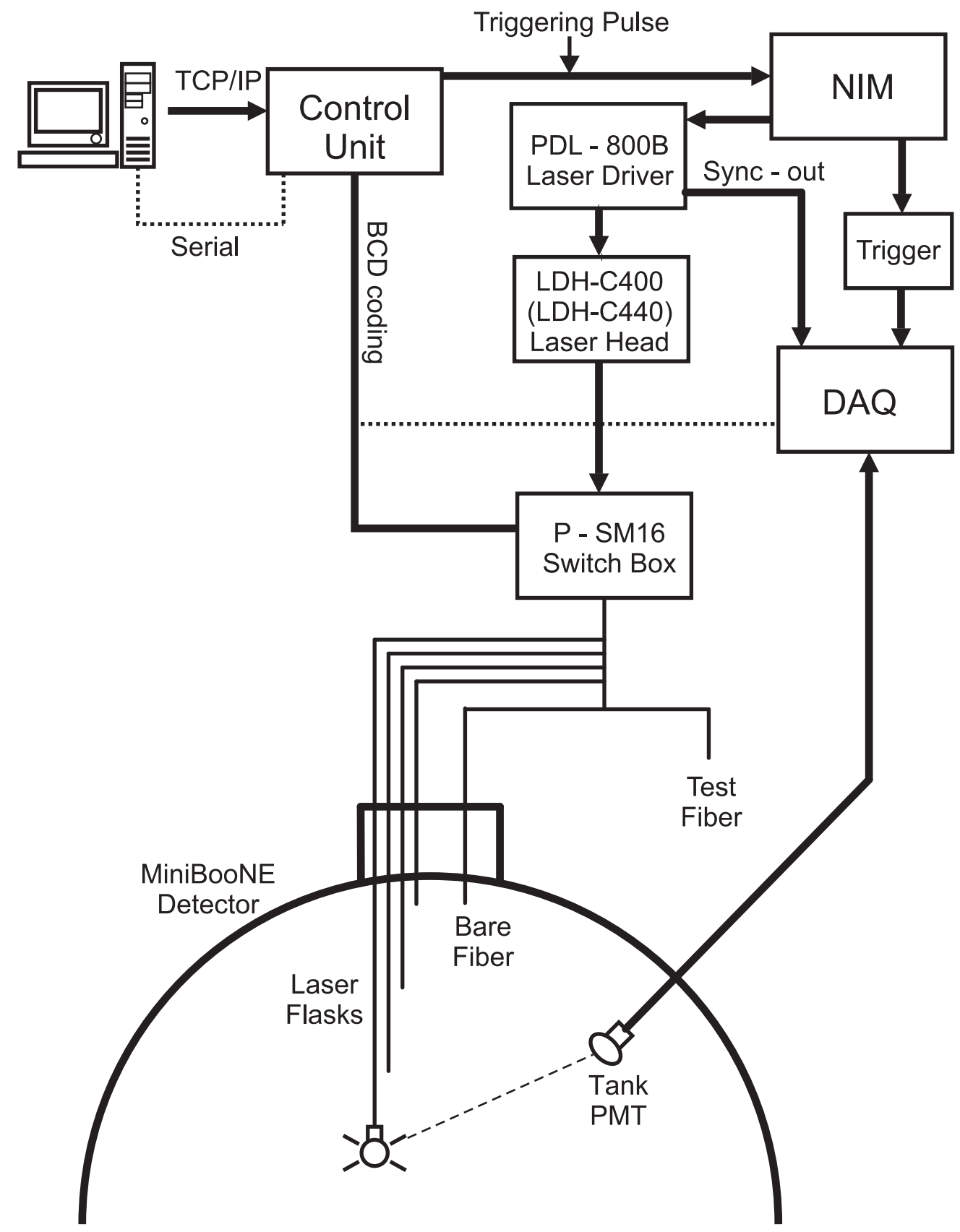

Figure 3.15: Schematic of the MiniBooNE laser calibration system.From Ref. [87] 


\subsubsection{Laser Calibration System}

The laser calibration system consists of a pulsed diode laser and four dispersion flasks, installed at various locations in the detector (see Fig. 3.15). Optical fibers connect the laser to the dispersion flasks through which short light pulses ( $<1 \mathrm{~ns})$ with a peak wavelength of $397 \mathrm{~nm}$ is transmitted. The dispersion flasks, each $10 \mathrm{~cm}$ in diameter, are filled with Ludox colloidal silica for uniform light dispersion. In addition to the four flasks there is also a bare optical fiber that emits light in a cone of about $10^{\circ}$ opening angle illuminating PMTs in a small circle near the bottom of the detector.

The primary purpose of the laser system is to quantify and monitor individual PMT performance parameters. The raw charge and time information for each PMT, as described in the previous section, are converted to calibrated charge and time using the information provided by the analysis of the laser calibration data. This quantities, called calibration constants (gains and time offsets, respectively), are used to account for individual differences in the charge and time response among the PMTs.

In addition, the laser system also allows for in situ monitoring of the oil parameters over the lifetime of the experiment.

\subsubsection{Muon Calibration System}

Muon decay is a well known physical process which results in a neutrino-antineutrino pair and an electron, with a decay time of $2.2 \mu$ s (in vacuum):

$$
\mu^{-} \rightarrow e^{-}+\bar{\nu}_{e}+\nu_{\mu} \quad \text { or } \quad \mu^{+} \rightarrow e^{+}+\nu_{e}+\bar{\nu}_{\mu} .
$$

For decays at rest, the resulting electron (also called a Michel electron) has a continuous energy spectrum of a precisely known shape

$$
f(E)=x^{2}(x-1) \quad \text { where } \quad x=\frac{E}{E_{\text {end }}}
$$


with an end-point energy of $E_{\text {end }}=52.8 \mathrm{MeV}$ (half the muon mass if we neglect the electron mass) - see Fig. 3.17. MiniBooNE uses a muon calibration system where the muon decay spectrum has been used extensively to tune and verify not only the Monte Carlo simulations, but also the event reconstruction algorithms.

The muon calibration system consists of a scintillator hodoscope placed above the detector and seven scintillator cubes deployed within the detector. The hodoscope has two layers of plastic scintillator, each with an $x$ and $y$ plane, to determine the position and directions of muons entering the detector. The scintillator cubes are placed at various distances from the optical barrier. The cubes are connected by optical fibers to a 1 inch PMT for readout. A muon entering the detector passes through the tracker and stops in a scintillation cube and decays. The Cherenkov light produced by the muon is seen by the PMTs as well as the scintillation cube. The decay electrons are also seen by the tank PMTs. Those events where the location and momentum of the muon and the origin of the electron can be determined by the muon hodoscope help in tuning and verifying the reconstruction algorithms. 


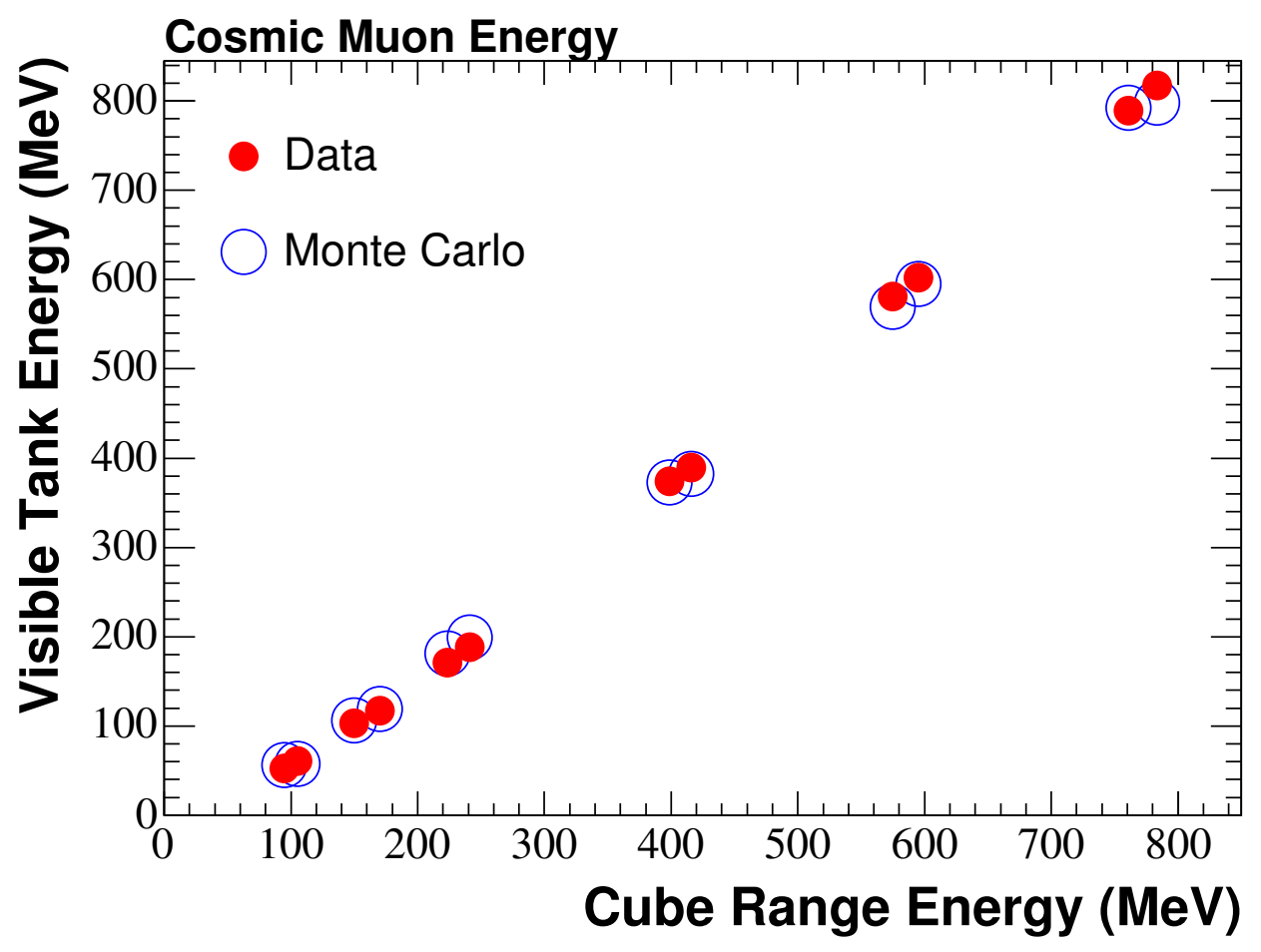

Figure 3.16: Muon energy calibration with the cosmic muon calibration system. The $x$-axis shows the energy calculated using the stopping muon range tables and the $y$-axis shows the reconstructed energy assuming an electron hypothesis (no dedicated muon fitters were available when these calibration measurements were first performed. The size of the circles indicates the size of the error bars. From Ref [92]

\subsubsection{Michel Electron Calibration}

As mentioned before, the muon decay is a well studied process with a known spectrum which is used in the muon calibration system in MiniBooNE. Another aspect of muon decay is the fact that resultant Michel electrons have a well-known end point energy, $E_{\text {end }}=52.8 \mathrm{MeV}$, which is used to calibrate electrons.

In MiniBooNE a muon candidate event generates more than 200 hits in the main tank and more than 6 hits in the veto PMTs. The subsequent Michel electron is recorded after an average delay of about $2.2 \mu$ s with a main tank multiplicity below 200 and veto hit multiplicity below 6. Figure 3.17 shows the observed spectrum of Michel electron candidate events together with the best fit to the data. The smearing in the spectrum is due to the limited energy resolution. From a fit around the end point the energy resolution was 
determined to be about $14.8 \%$ in this energy regime.

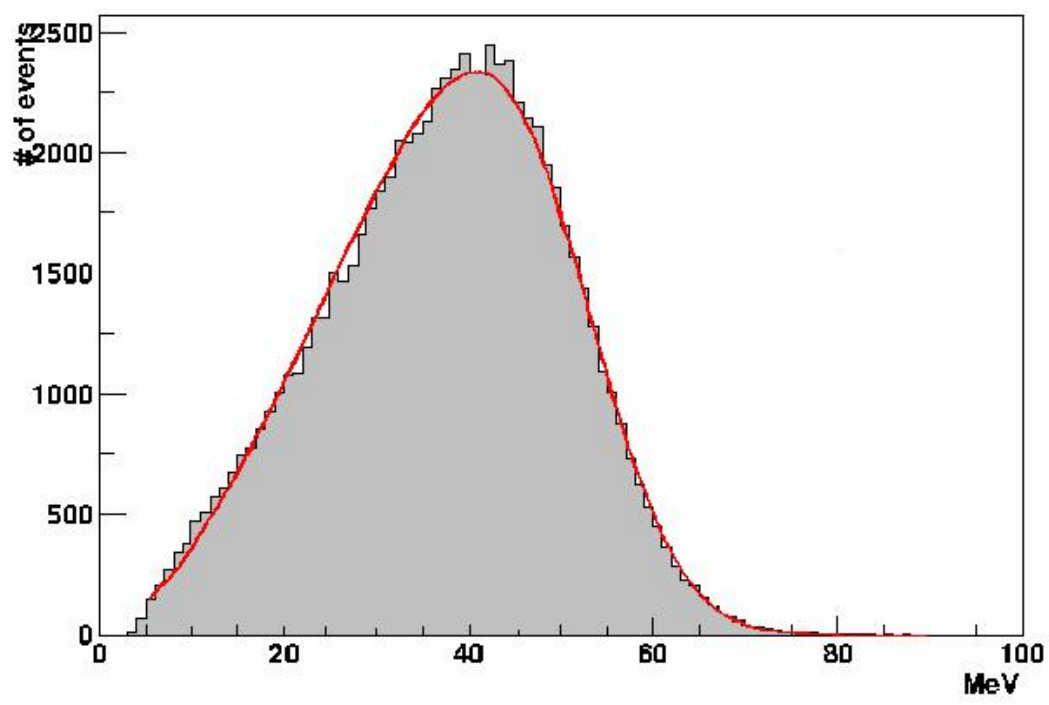

Figure 3.17: Michel electron reconstructed energy spectrum (gray) and the best fit (red), obtained by smearing the Michel electron kinetic energy spectrum with a Gaussian of width proportional to $\sqrt{E}$.

\subsubsection{Neutral Pion Mass Calibration}

The reconstructed $\pi^{0}$ mass (referred to as $M_{\gamma \gamma}$ henceforth), as calculated from the beaminduced neutral current $\pi^{0}$ events, is another calibration employed in MiniBooNE. The $\pi^{0}$ meson (with a rest mass of $135 \mathrm{MeV}$ ) decays after being produced almost immediately (decay time $\left.8.4 \times 10^{-17} \mathrm{~s}\right)$, via the following modes:

$$
\begin{array}{cc}
\pi^{0} \rightarrow \gamma+\gamma & \text { (branching fraction 98.987\%) } \\
\pi^{0} \rightarrow \gamma+e^{-}+e^{+} & \text {(branching fraction 1.198\%). }
\end{array}
$$

The resulting gammas convert into electron-positron pairs after travelling $\sim 0.5 \mathrm{~m}$ and produce an electromagnetic shower in the detector emitting both Cherenkov and scintillation light. The electrons (assumed massless) also retain the same direction as the original gamma as the gamma is massless. 
The $\pi^{0} M_{\gamma \gamma}$ distribution is used to calibrate photons in the energy range between $50 \mathrm{MeV}$ and $350 \mathrm{MeV}$. Fig. 3.18 shows the comparison between the reconstructed $M_{\gamma \gamma}$ distributions for data and Monte Carlo in various $\pi^{0}$ momentum bins. Details of NC $\pi^{0}$ sample selection and reconstruction (including 4-vertex, directions of the two gammas, their conversion lengths and energies) are given in Ref. [93].
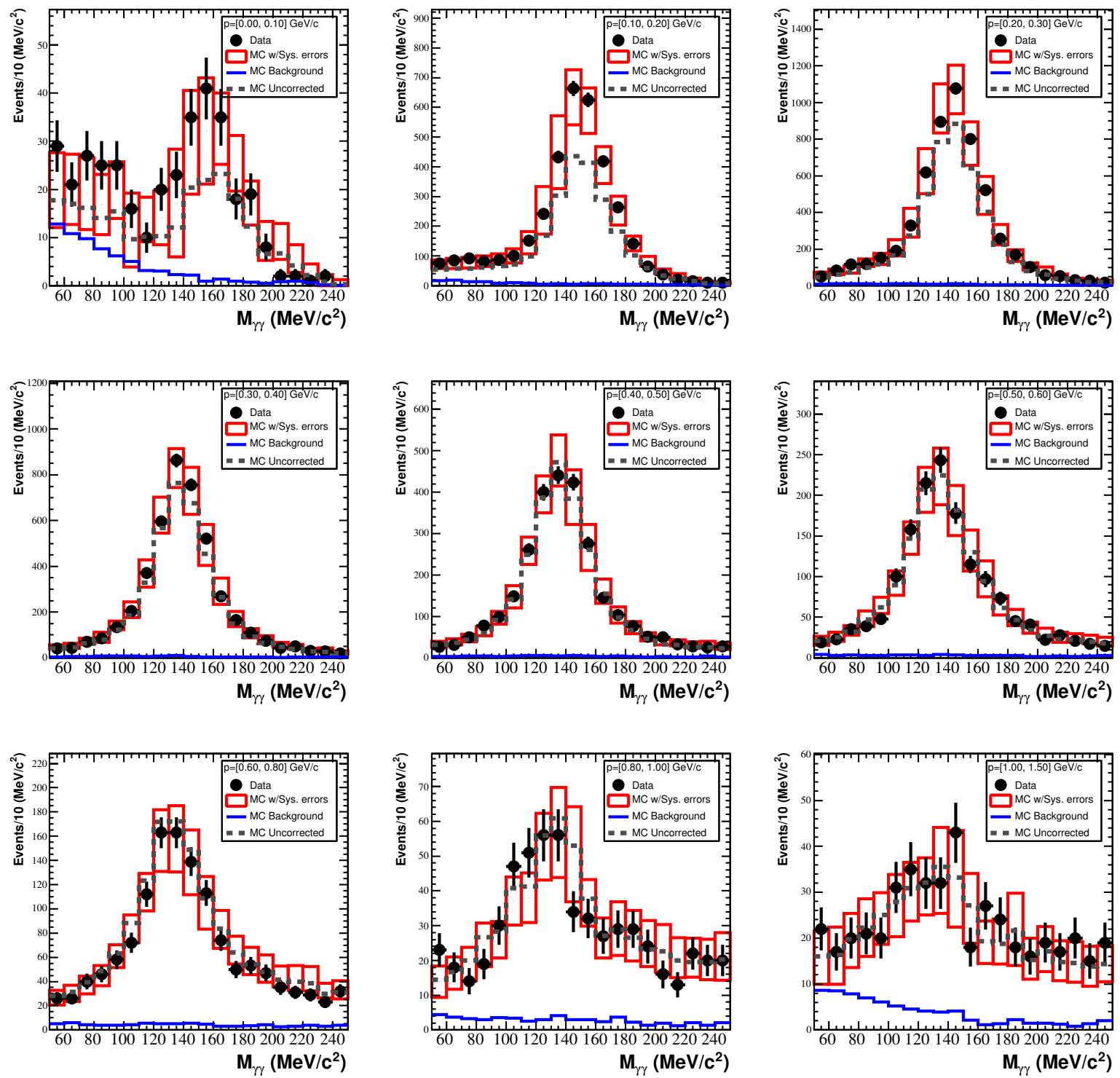

Figure 3.18: NC $\pi^{0}$ mass calibration. The plots show reconstructed $M_{\gamma \gamma}$ distributions for data(black points with statistical error bars) along with the uncorrected(dashed histogram) and corrected MC (red) with systematic errors in bins of reconstructed $\pi^{0}$ momentum from $0 \mathrm{GeV} / \mathrm{c}$ to $1.5 \mathrm{GeV} / \mathrm{c}$. Also shown are the MC estimate for $\mathrm{NC} \pi^{0}$ backgrounds (blue). From Ref. [93] 


\subsection{The MiniBooNE Cross section model}

Neutrino interaction rates, products, and their kinematics in the MiniBooNE detector are predicted using a neutrino event generator which is based on the NUANCE v3 [94], but has been customized to the MiniBooNE experiment. The various parameters within the generator are tuned to the current best-known cross section information. It accepts as input the neutrino flux prediction described in $\S 3.4$, as well as the detector target material and geometry. The mineral oil target is simulated as $\mathrm{CH}_{2}$ with a density of $0.845 \mathrm{~g} / \mathrm{cm}^{3}$. NUANCE can simulate 99 distinct neutrino/antineutrino interactions, in a wide energy range (100 $\mathrm{MeV}$ to $\left.10^{3} \mathrm{GeV}\right)$. Tables 3.2 and 3.3 list the interaction processes simulated by NUANCE along with the channel numbers. Table 3.4 lists the cross section parameters and the assigned uncertainties used in the MiniBooNE cross section model.

The following sections describe in detail the interaction channels important for this analysis:

\subsubsection{Neutral Current Elastic Scattering}

Antineutrino NCE scattering, the signal for this analysis, and the neutrino NCE scattering background, both correspond to channel \#2 in NUANCE. The neutrino scattering off of six bound nucleons in the carbon atom and two free protons in hydrogen atom of the MiniBooNE target $\mathrm{CH}_{2}$ is modelled by different methods in NUANCE. For the scattering off free protons, the Llewellyn-Smith formalism [61] (described in §2.2) is used, while for bound nucleons the relativistic Fermi gas (RFG) model of Smith and Moniz [95] is used.

The vector form factors are taken from Ref. [96], while other form factors are customized as follows: the isoscalar form factor is taken to be $\Delta s=0$, while the axial vector mass is

- $M_{A}=1.23 \mathrm{GeV}$ for scattering off bound nucleons (taken from MiniBooNE CCQE measurement [59]),

- $M_{A}=1.13 \mathrm{GeV}$ for scattering off free protons. This corresponds to the average between 
the value obtained from deuterium-based CCQE measurements $\left(M_{A}=1.03 \mathrm{GeV}\right)$ and the MiniBooNE CCQE measurement above.

\subsubsection{Charged Current Elastic Scattering}

NUANCE channel\#1 corresponds to both neutrino and antineutrino CCQE interactions which are a background for this analysis. The neutrino CCQE interaction happens off neutrons $\left(\nu_{\mu}+n \rightarrow \mu^{-}+p\right)$ in the bound carbon atom, hence the Smith-Moniz [95] formalism is employed, whereas the antineutrino CCQE happens off protons $\left(\bar{\nu}_{\mu}+p \rightarrow \mu^{+}+n\right)$ which are both free $(\mathrm{H}$ atom $)$ and bound $(\mathrm{C}$ atom), hence a combination of the Llewellyn-Smith and Smith-Moniz formalism is used as in the case of NCE scattering. The CCQE scattering model is similar to the NCE scattering model described in $\S 2.2$ with the difference that the form factors do not include additional coupling factors and contributions from strange quarks.

The form factors have the same values as in the NCE case, i.e., the axial vector mass $M_{A}=1.23 \mathrm{GeV}$ for the bound nucleons and $M_{A}=1.13 \mathrm{GeV}$ for the free nucleons.

Finally, a Pauli blocking scaling parameter $\kappa=1.022$ is used here, which modifies the shape of the CCQE cross section at low values of momentum transfer. See ref [59] for details. 


\begin{tabular}{|c|c|c|}
\hline$\#$ & $\begin{array}{l}\mathrm{CC} / \\
\mathrm{NC}\end{array}$ & Reaction \\
\hline \multicolumn{3}{|c|}{$\begin{array}{l}\text { Cabibbo-allowed } \\
\text { quasi-elastic scattering from } \\
\text { nucleons }\end{array}$} \\
\hline 1 & $\mathrm{CC}$ & $\begin{array}{l}\nu_{\mu} \mathrm{n} \rightarrow \mu^{-} \mathrm{p} \\
\left(\bar{\nu}_{\mu} \mathrm{p} \rightarrow \mu^{+} \mathrm{n}\right)\end{array}$ \\
\hline \multicolumn{3}{|c|}{$\begin{array}{l}\text { (Quasi-)elastic scattering } \\
\text { from nucleons }\end{array}$} \\
\hline 2 & $\mathrm{NC}$ & $\begin{array}{l}\nu_{\mu} \mathrm{n} \rightarrow \nu_{\mu} \mathrm{n} \\
\left(\bar{\nu}_{\mu} \mathrm{n} \rightarrow \bar{\nu}_{\mu} \mathrm{n}\right) \\
\nu_{\mu} \mathrm{p} \rightarrow \nu_{\mu} \mathrm{p} \\
\left(\bar{\nu}_{\mu} \mathrm{p} \rightarrow \bar{\nu}_{\mu} \mathrm{p}\right)\end{array}$ \\
\hline \multicolumn{3}{|c|}{$\begin{array}{l}\text { Resonant single pion } \\
\text { production }\end{array}$} \\
\hline 3 & $\mathrm{CC}$ & $\nu_{\mu} \mathrm{p} \rightarrow \mu^{-} \mathrm{p} \pi^{+}$ \\
\hline 4 & $\mathrm{CC}$ & $\nu_{\mu} \mathrm{n} \rightarrow \mu^{-} \mathrm{p} \pi^{0}$ \\
\hline 5 & $\mathrm{CC}$ & $\nu_{\mu} \mathrm{n} \rightarrow \mu^{-} \mathrm{n} \pi^{+}$ \\
\hline 6 & $\mathrm{NC}$ & $\nu_{\mu} \mathrm{p} \rightarrow \nu_{\mu} \mathrm{p} \pi^{0}$ \\
\hline 7 & $\mathrm{NC}$ & $\nu_{\mu} \mathrm{p} \rightarrow \nu_{\mu} \mathrm{n} \pi^{+}$ \\
\hline 8 & $\mathrm{NC}$ & $\nu_{\mu} \mathrm{n} \rightarrow \nu_{\mu} \mathrm{n} \pi^{0}$ \\
\hline 9 & $\mathrm{NC}$ & $\nu_{\mu} \mathrm{n} \rightarrow \nu_{\mu} \mathrm{p} \pi^{-}$ \\
\hline & -16 & $\begin{array}{l}\text { Corresponding } \bar{\nu}_{\mu} \\
\text { processes }\end{array}$ \\
\hline \multicolumn{3}{|c|}{$\begin{array}{l}\text { Multi-pion resonant } \\
\text { processes }\end{array}$} \\
\hline 17 & $\mathrm{CC}$ & $\nu_{\mu} \mathrm{p} \rightarrow \mu^{-} \Delta^{+} \pi^{+}$ \\
\hline 18 & $\mathrm{CC}$ & $\nu_{\mu} \mathrm{p} \rightarrow \mu^{-} \Delta^{++} \pi^{0}$ \\
\hline 19 & $\mathrm{CC}$ & $\nu_{\mu} \mathrm{n} \rightarrow \mu^{-} \Delta^{+} \pi^{0}$ \\
\hline 20 & $\mathrm{CC}$ & $\nu_{\mu} \mathrm{n} \rightarrow \mu^{-} \Delta^{0} \pi^{+}$ \\
\hline 21 & $\mathrm{CC}$ & $\nu_{\mu} \mathrm{n} \rightarrow \mu^{-} \Delta^{++} \pi^{-}$ \\
\hline 22 & $\mathrm{NC}$ & $\nu_{\mu} \mathrm{p} \rightarrow \nu_{\mu} \Delta^{+} \pi^{0}$ \\
\hline 23 & $\mathrm{NC}$ & $\nu_{\mu} \mathrm{p} \rightarrow \nu_{\mu} \Delta^{0} \pi^{+}$ \\
\hline 24 & $\mathrm{NC}$ & $\nu_{\mu} \mathrm{p} \rightarrow \nu_{\mu} \Delta^{++} \pi^{-}$ \\
\hline
\end{tabular}

\begin{tabular}{|c|c|c|}
\hline$\#$ & $\begin{array}{l}\mathrm{CC} / \\
\mathrm{NC}\end{array}$ & Reaction \\
\hline 25 & $\mathrm{NC}$ & $\nu_{\mu} \mathrm{n} \rightarrow \nu_{\mu} \Delta^{+} \pi^{-}$ \\
\hline 26 & $\mathrm{NC}$ & $\nu_{\mu} \mathrm{n} \rightarrow \nu_{\mu} \Delta^{0} \pi^{0}$ \\
\hline 27 & $\mathrm{NC}$ & $\nu_{\mu} \mathrm{n} \rightarrow \nu_{\mu} \Delta^{-} \pi^{+}$ \\
\hline \multicolumn{2}{|c|}{$28-38$} & $\begin{array}{l}\text { Corresponding } \bar{\nu}_{\mu} \\
\text { processes }\end{array}$ \\
\hline 39 & $\mathrm{CC}$ & $\nu_{\mu} \mathrm{p} \rightarrow \mu^{-} \mathrm{p} \rho^{+}(770)$ \\
\hline 40 & $\mathrm{CC}$ & $\nu_{\mu} \mathrm{n} \rightarrow \mu^{-} \mathrm{p} \rho^{0}(770)$ \\
\hline 41 & $\mathrm{CC}$ & $\nu_{\mu} \mathrm{n} \rightarrow \mu^{-} \mathrm{n} \rho^{+}(770)$ \\
\hline 42 & $\mathrm{NC}$ & $\nu_{\mu} \mathrm{p} \rightarrow \nu_{\mu} \mathrm{p} \rho^{0}(770)$ \\
\hline 43 & $\mathrm{NC}$ & $\nu_{\mu} \mathrm{p} \rightarrow \nu_{\mu} \mathrm{n} \rho^{+}(770)$ \\
\hline 44 & $\mathrm{NC}$ & $\nu_{\mu} \mathrm{n} \rightarrow \nu_{\mu} \mathrm{n} \rho^{0}(770)$ \\
\hline 45 & $\mathrm{NC}$ & $\nu_{\mu} \mathrm{n} \rightarrow \nu_{\mu} \mathrm{p} \rho^{-}(770)$ \\
\hline \multicolumn{2}{|c|}{$46-52$} & $\begin{array}{l}\text { Corresponding } \bar{\nu}_{\mu} \\
\text { processes }\end{array}$ \\
\hline 53 & $\mathrm{CC}$ & $\nu_{\mu} \mathrm{p} \rightarrow \mu^{-} \Sigma^{+} \mathrm{K}^{+}$ \\
\hline 54 & $\mathrm{CC}$ & $\nu_{\mu} \mathrm{n} \rightarrow \mu^{-} \Sigma^{0} \mathrm{~K}^{+}$ \\
\hline 55 & $\mathrm{CC}$ & $\nu_{\mu} \mathrm{n} \rightarrow \mu^{-} \Sigma^{+} \mathrm{K}^{0}$ \\
\hline 56 & $\mathrm{NC}$ & $\nu_{\mu} \mathrm{p} \rightarrow \nu_{\mu} \Sigma^{0} \mathrm{~K}^{+}$ \\
\hline 57 & $\mathrm{NC}$ & $\nu_{\mu} \mathrm{p} \rightarrow \nu_{\mu} \Sigma^{+} \mathrm{K}^{0}$ \\
\hline 58 & $\mathrm{NC}$ & $\nu_{\mu} \mathrm{n} \rightarrow \nu_{\mu} \Sigma^{0} \mathrm{~K}^{0}$ \\
\hline 59 & $\mathrm{NC}$ & $\nu_{\mu} \mathrm{n} \rightarrow \nu_{\mu} \Sigma^{-} \mathrm{K}^{+}$ \\
\hline \multicolumn{2}{|c|}{$60-66$} & $\begin{array}{l}\text { Corresponding } \bar{\nu}_{\mu} \\
\text { processes }\end{array}$ \\
\hline 67 & $\mathrm{CC}$ & $\nu_{\mu} \mathrm{n} \rightarrow \mu^{-} \mathrm{p} \eta$ \\
\hline 68 & $\mathrm{NC}$ & $\nu_{\mu} \mathrm{p} \rightarrow \nu_{\mu} \mathrm{p} \eta$ \\
\hline 69 & $\mathrm{NC}$ & $\nu_{\mu} \mathrm{n} \rightarrow \nu_{\mu} \mathrm{n} \eta$ \\
\hline \multicolumn{2}{|c|}{$70-72$} & $\begin{array}{l}\text { Corresponding } \bar{\nu}_{\mu} \\
\text { processes }\end{array}$ \\
\hline 73 & $\mathrm{CC}$ & $\nu_{\mu} \mathrm{n} \rightarrow \mu^{-} \mathrm{K}^{+} \Lambda$ \\
\hline 74 & $\mathrm{NC}$ & $\nu_{\mu} \mathrm{p} \rightarrow \nu_{\mu} \mathrm{K}^{+} \Lambda$ \\
\hline 75 & $\mathrm{NC}$ & $\nu_{\mu} \mathrm{n} \rightarrow \nu_{\mu} \mathrm{K}^{0} \Lambda$ \\
\hline
\end{tabular}

Table 3.2: Processes available within NUANCE. The numbers in the leftmost column indicate the assigned reaction code (channel number) in NUANCE (continued on the next page). 


\begin{tabular}{|c|c|l|}
\hline$\#$ & $\begin{array}{l}\text { CC } \\
\text { NC }\end{array}$ & Reaction \\
\hline \hline \multicolumn{2}{|c|}{$76-78$} & $\begin{array}{l}\text { Corresponding } \bar{\nu}_{\mu} \\
\text { processes }\end{array}$ \\
\hline 79 & $\mathrm{CC}$ & $\nu_{\mu} \mathrm{n} \rightarrow \mu^{-} \mathrm{p} \pi^{+} \pi^{-}$ \\
\hline 80 & $\mathrm{CC}$ & $\nu_{\mu} \mathrm{n} \rightarrow \mu^{-} \mathrm{p} \pi^{0} \pi^{0}$ \\
\hline 81 & $\mathrm{NC}$ & $\nu_{\mu} \mathrm{p} \rightarrow \nu_{\mu} \mathrm{p} \pi^{+} \pi^{-}$ \\
\hline 82 & $\mathrm{NC}$ & $\nu_{\mu} \mathrm{p} \rightarrow \nu_{\mu} \mathrm{p} \pi^{0} \pi^{0}$ \\
\hline 83 & $\mathrm{NC}$ & $\nu_{\mu} \mathrm{n} \rightarrow \nu_{\mu} \mathrm{n} \pi^{+} \pi^{-}$ \\
\hline 84 & $\mathrm{NC}$ & $\nu_{\mu} \mathrm{n} \rightarrow \nu_{\mu} \mathrm{n} \pi^{0} \pi^{0}$ \\
\hline $85-90$ & $\begin{array}{l}\text { Corresponding } \bar{\nu}_{\mu} \\
\text { processes }\end{array}$ \\
\hline \multicolumn{2}{|c|}{ Deep Inelastic Scattering } \\
\hline 91 & $\mathrm{CC}$ & $\nu_{\mu} \mathrm{N} \rightarrow \mu \mathrm{X}$ \\
\hline 92 & $\mathrm{NC}$ & $\nu_{\mu} \mathrm{N} \rightarrow \nu_{\mu} \mathrm{X}$ \\
\hline $93-94$ & Unused \\
\hline 95 & CC & $\begin{array}{l}\text { Cabibbo-supp. QE } \\
\text { hyperon production: }\end{array}$ \\
& & $\begin{array}{l}\bar{\nu}_{\mu} \mathrm{p} \rightarrow \mu^{+} \Lambda \\
\bar{\nu}_{\mu} \mathrm{n} \rightarrow \mu^{+} \Sigma^{-} \\
\bar{\nu}_{\mu} \mathrm{p} \rightarrow \mu^{+} \Sigma^{0}\end{array}$ \\
\hline
\end{tabular}

\begin{tabular}{|c|c|c|}
\hline$\#$ & $\begin{array}{l}\mathrm{CC} / \\
\mathrm{NC}\end{array}$ & Reaction \\
\hline \multicolumn{3}{|c|}{$\begin{array}{l}\text { Coherent / diffractive } \pi \\
\text { production }\end{array}$} \\
\hline 96 & $\mathrm{NC}$ & $\begin{array}{l}\nu_{\mu} \mathrm{A} \rightarrow \nu_{\mu} \mathrm{A} \pi^{0} \\
\left(\bar{\nu}_{\mu} \mathrm{A} \rightarrow \bar{\nu}_{\mu} \mathrm{A} \pi^{0}\right)\end{array}$ \\
\hline 97 & $\mathrm{CC}$ & $\begin{array}{l}\nu_{\mu} \mathrm{A} \rightarrow \mu^{-} \mathrm{A} \pi^{+} \\
\left(\bar{\nu}_{\mu} \mathrm{A} \rightarrow \mu^{+} \mathrm{A} \pi^{-}\right)\end{array}$ \\
\hline \multicolumn{3}{|c|}{$\nu-\mathrm{e}$ elastic scattering } \\
\hline 98 & - & $\begin{array}{l}\nu_{\mu} \mathrm{e} \rightarrow \nu_{\mu} \mathrm{e} \\
\left(\bar{\nu}_{\mu} \mathrm{e} \rightarrow \bar{\nu}_{\mu} \mathrm{e}\right)\end{array}$ \\
\hline \multicolumn{3}{|c|}{$\nu-\mathrm{e}$ inverse $\mu$ decay } \\
\hline 99 & $\mathrm{CC}$ & $\nu_{\mu} \mathrm{e} \rightarrow \mu^{-} \nu_{\mathrm{e}}$ \\
\hline
\end{tabular}

Table 3.3: Processes available within NUANCE (continued from the previous page). The numbers in the leftmost column indicate the assigned reaction code (channel number) in NUANCE.

Parameter Value Uncertainty

$M_{A}$ for $\mathrm{QE}$ events on carbon

Binding energy for carbon

\begin{tabular}{cc}
$1.2341 \mathrm{GeV}$ & $0.077 \mathrm{GeV}$ \\
$34.0 \mathrm{MeV}$ & $9.0 \mathrm{MeV}$ \\
$220.0 \mathrm{MeV}$ & $30.0 \mathrm{MeV}$ \\
0.0 & 0.1 \\
$1.1 \mathrm{GeV}$ & $0.27 \mathrm{GeV}$ \\
$1.3 \mathrm{GeV}$ & $0.52 \mathrm{GeV}$ \\
1.302 & 0.14 \\
1.00 & 0.12 \\
1.00 & 0.25 \\
1.0220 & 0.0205 \\
$1.030 \mathrm{GeV}$ & $0.275 \mathrm{GeV}$ \\
1.00 & 0.14 \\
$1.13 \mathrm{GeV}$ & $0.10 \mathrm{GeV}$ \\
\hline
\end{tabular}

Table 3.4: Cross-section parameters and their 1- $\sigma$ uncertainties used in the MiniBooNE MC. Here the abbreviations are: $\mathrm{QE}=$ quasi-elastic, $\mathrm{NC}=$ neutral current, and $\mathrm{CC}=$ charge current. 


\subsubsection{Neutral-Current Single Pion Production}

The neutral current single pion production channels (channels 6-9 for neutrinos and 10-16 for antineutrinos) are a major background for this analysis. In neutrino-nucleus interactions single pion production happens when a nucleon is excited to a resonant state and subsequently decays to a pion and a nucleon. The pions may experience absorption in the nucleus through final state interactions (FSI) in which case the final product of the interaction is a nucleon, similar to the NCE interaction. MiniBooNE has tuned NUANCE to assign a probability of about $20 \%$ that the outgoing pion is absorbed.

The resonant pion production is modelled in NUANCE using the Rein and Sehgal model [97]. At MiniBooNE energies $(\sim 1 \mathrm{GeV})$ the interaction is dominated by the $\Delta(1232)$ resonance, although higher mass resonances are included in the simulation. The form factors are assumed to be identical to those used in NCE and CCQE interaction, with the exception of the axial vector mass, where we take $M_{A}=1.1 \mathrm{GeV}$ - see Ref. [98].

\subsubsection{Nuclear effects}

In the case of bound nucleons, nuclear effects modify the above interaction rates. NUANCE employs the RFG model of Smith and Moniz to incorporate these effects. In the RFG model, target nucleons are assumed to have a uniform momentum density distribution up to a cutoff Fermi momentum of $p_{F}=220 \mathrm{MeV}$. A scattering interaction is only allowed if the outgoing nucleon has a momentum greater than $p_{F}$. This effect is called Pauli blocking and suppresses the cross section at low values of the momentum transfer.

The low $Q^{2}$ regime, where nuclear effects are dominant, is untested by other experiments and the RFG model of Smith and Moniz strain to match the observed data. Hence in MiniBooNE the Pauli blocking parameter is scaled by a scaling factor $\kappa=1.022$, whereas in the RFG model of Smith and Moniz $\kappa$ is unity - see Ref. [99] for details.

Last of the nuclear effects are final state interactions (FSI). This internuclear effect refers to the strong interaction the recoil hadrons experience due to the surrounding nucleons inside 
the carbon nucleus. NUANCE accounts for this effect based on models of nuclear density and Fermi momentum, and generating new final state particles at each step if the MC determines that a FSI has occurred.

\subsubsection{Dirt Interactions}

NUANCE also simulates the neutrino interactions with the material surrounding the detector and the detector material (collectively called as "dirt" interactions). The interactions are simulated the same way as the in-tank interactions however the cross sections are reweighted according to the density of the material relative to that of mineral oil.

\subsection{The MiniBooNE Detector Monte Carlo}

The MiniBooNE neutrino event generator (NUANCE) outputs a set of neutrino-generated particles and channels. This information is used by the detector simulation to model particle propagation, the resulting light emission and propagation, and the PMT response. MiniBooNE uses the GEANT3 simulation software [100] in conjunction with a customized optical model to simulate interactions in the detector. 


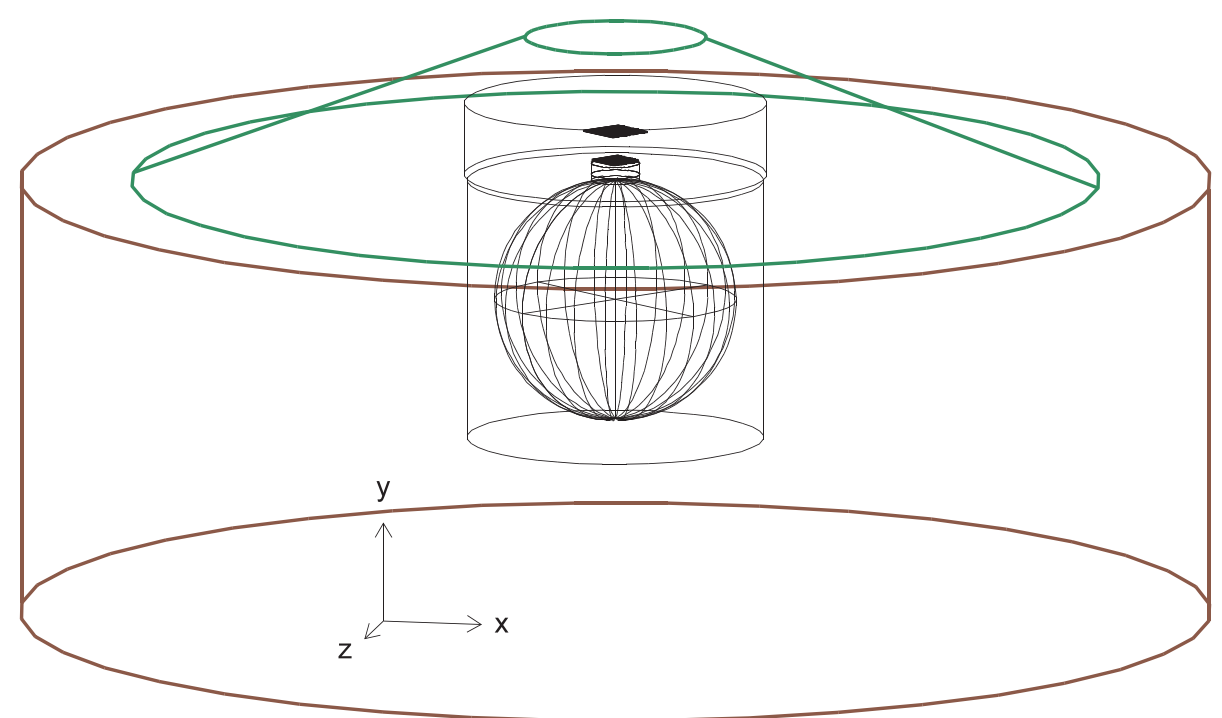

Figure 3.19: The geometric domain of the MiniBooNE GEANT3 simulation. The dirt region and the overburden are indicated by the thick-lined cylinder and the conical frustum, the thinlined cylinders indicate the electronics room and vault, the sphere and the top-hat determine the detector tank and the tophat. From Ref. [101]

The geometric domain of simulation includes the tank, surrounding dirt and the the vault housing electronics above the tank. In the simulation, the mineral oil is assumed to be $\mathrm{CH}_{2}$ with a density of $0.845 \mathrm{~g} / \mathrm{cm}^{3}$. The details of the PMT structure and calibration devices like the scintillator cubes and laser flasks are also included. The dirt surrounding the detector is assumed to be composed of a mixture of silicon, aluminum, and oxygen with respective densities.

The default GEANT3 tracking algorithm is employed to propagate the particles resulting from the neutrino interactions. Some modifications to the standard routines include an improved model for Dalitz decay $\left(\pi^{0} \rightarrow e^{+} e^{-} \gamma\right)$, muon decay $(\mu \rightarrow e \nu \nu)$, and the possibility of $\mu^{-}$capture by carbon. The default hadronic interaction package, GFLUKA, was replaced with GCALOR [102].

\subsubsection{The MiniBooNE Optical Model}

MiniBooNE has developed a custom made optical model to characterize the optical photons which are produced by the charged particles traversing the detector. The MiniBooNE optical 
model characterizes both the production of photons and propagation within the detector until it reaches a PMT. The MiniBooNE PMTs are sensitive to photons over the wavelength range of 280-640 nm, as shown in Fig. 3.20 below.

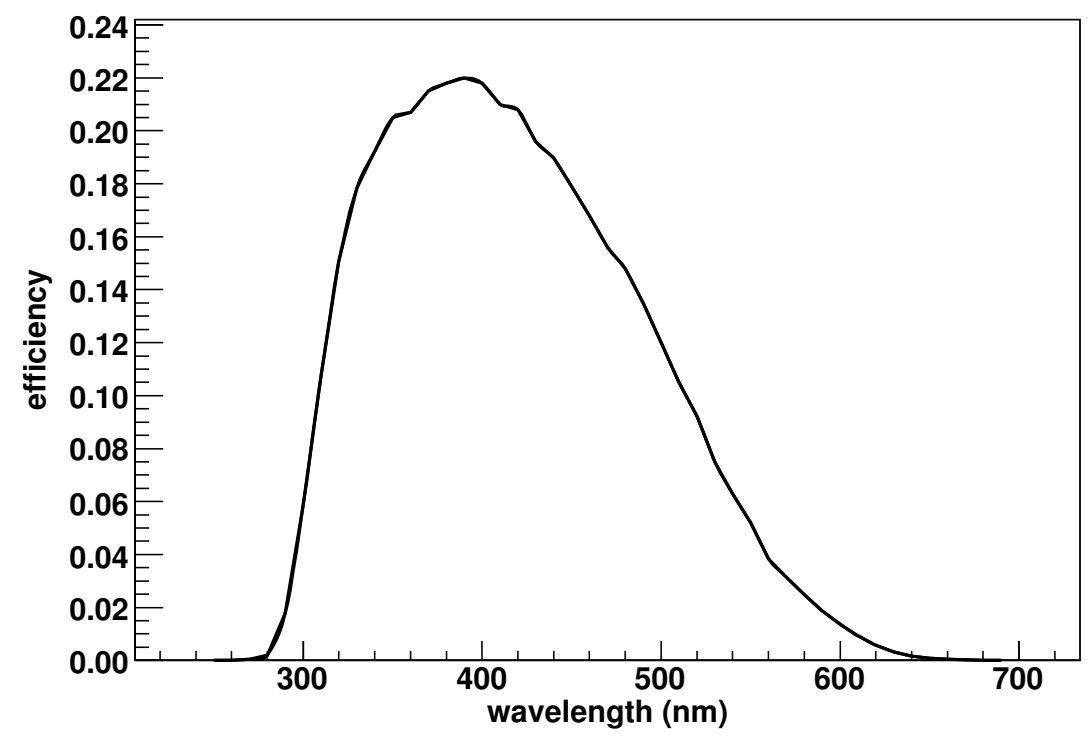

Figure 3.20: Quantum efficiency of R5912 PMT obtained from Hamamatsu measurements.

\subsubsection{Light production}

Light production in MiniBooNE occurs through Cherenkov radiation, scintillation, and fluorescence, as described below:

- Cherenkov radiation

Whenever a charged particle moves with a velocity faster than the velocity of light in that medium it emits Cherenkov light. Cherenkov radiation is prompt and directional. The angle between the particle track and the emitted photons is governed by the formula:

$$
\cos \theta_{c}=\frac{1}{\beta n}
$$

where $n$ is the refractive index of the medium and $\beta$ is the particle velocity with respect to the speed of light, $\beta=v / c$. The rate of photon emission as a function of wavelength 
is given by:

$$
\frac{d N}{d x d \omega}=\alpha q^{2}\left[1-\frac{1}{\beta^{2} n^{2}(\omega)}\right],
$$

where $x$ is the path-length, $\omega$ is the photon frequency, $n$ is the refractive index, $\alpha$ is the fine structure constant, and $q$ is the particle charge.

Using Eq. (3.2), the particle velocity can be expressed through its kinetic energy, $T$,

$$
\cos \theta_{c e r}=\frac{1}{\beta n}=\frac{1}{n}\left[\frac{(T+m)^{2}}{(T+m)^{2}-m^{2}}\right]^{\frac{1}{2}},
$$

where $m$ is the rest mass of the particle. The minimum kinetic energy to produce Cherenkov light is obtained from $\beta=1 / n$, which yields for the Cherenkov kinetic energy threshold

$$
T_{C h}=m\left(\frac{n}{\sqrt{n^{2}-1}}-1\right) .
$$

Herewith, the Cherenkov threshold for protons in the MiniBooNE target oil is approximately $T_{C h} \simeq 350 \mathrm{MeV}$.

\section{- Scintillation and Fluorescence}

The ionization tracks of particles excite the electrons in the $\mathrm{CH}_{2}$ atom, the subsequent de-excitation results in the emission of energy in the form of photons. This is known as scintillation light and is delayed and isotropic in nature. When optical photons are responsible for excitation and de-excitation of the target molecules, as opposed to ionization tracks, the process is known as fluorescence.

The characteristics of scintillation and fluorescence (the wavelength spectra and emission spectra) are determined by the chemical composition of the oil, in particular the various flours and vitamin $\mathrm{E}$ added for oil preservation. The scintillation light yield is proportional to the particle ionization loss, while quenching effects reduce this light yield. Birk's law is used to convert ionization energy loss per unit length per unit 
medium density, $d E / d x$, to scintillation light production,

$$
\frac{d E_{s c i}}{d x}=\frac{\frac{d E}{d x}}{1+k_{B} \frac{d E}{d x}+k_{B}^{\prime}\left(\frac{d E}{d x}\right)^{2}}
$$

where the value of the coefficients $k_{B}=0.014 \mathrm{~g} \mathrm{~cm}^{2} \mathrm{MeV}^{-1}$ and $k_{B}^{\prime}=0$ were obtained empirically.
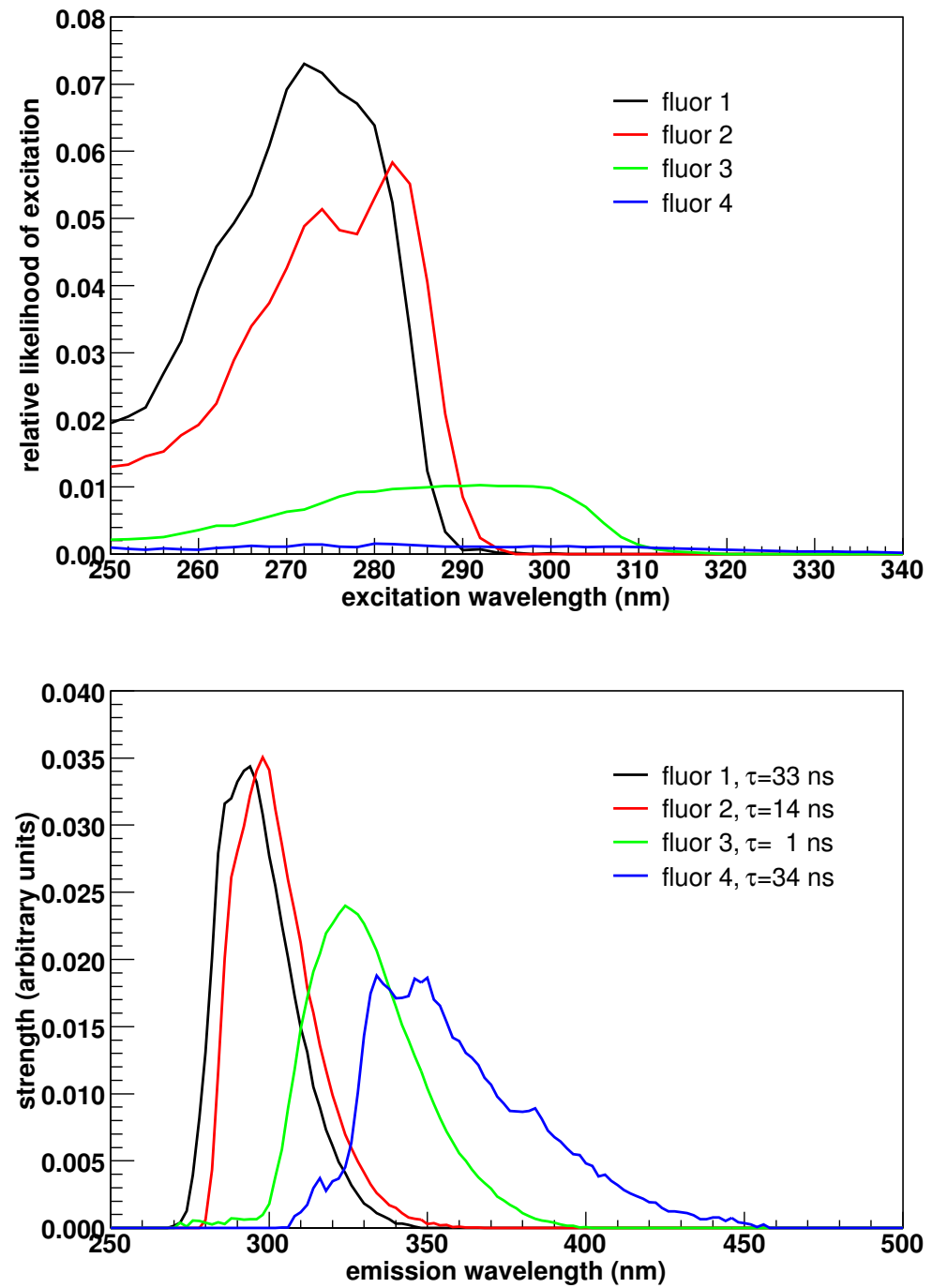

Figure 3.21: Fluors in MiniBooNE oil. (Top) Likelihood of photon excitation for the fluors in the MiniBooNE mineral oil. (Bottom) Emission probability and wavelength for the same fluors; also shown here are the lifetime for the corresponding emission photons. Figure from Ref. [93] 


\subsubsection{Light transmission}

The propagation or transmission of photons in MiniBooNE occurs through processes such as scattering, fluorescence, and absorption.

- Scattering

When an optical photon interacts with a target molecule and is deflected and changes its polarization state while keeping the same original wavelength, the process is known a s scattering. Scattering measurements in the MiniBooNE oil were done externally [103] and are due to Rayleigh and Raman scattering.

- Fluorescence

Fluorescence is when optical photons (created by both Cherenkov and scintillation) are absorbed and reemitted later by target molecules at a different wavelength and/or direction. Details of the measurements which was conducted at Johns Hopkin University are given in Ref.[104]

Of particular importance is the fluorescence due to ultraviolet (UV) photons. They have a wavelength less than $250 \mathrm{~nm}$ and are not registered by the PMTs, as the PMT quantum efficiency is zero below $280 \mathrm{~nm}$, as shown in Fig. 3.20. However, the UV photons may be absorbed and remitted with a photon spectrum above $250 \mathrm{~nm}$ with an attenuation length of the order $1-10 \mathrm{~cm}$.

In the MC simulation the original UV photons are not propagated; however the UV fluorescent photons are generated along the particle track similar to the scintillation photons.

- Absorption

The difference between the total extinction rate and the sum of attenuation due to scattering and fluorescence is attributed to the absorption of photons in oil. 
Figure 3.21 shows the excitation wavelength and the emission wavelength for the fluors in the MiniBooNE mineral oil. The extinction rate as a function of wavelength for the fluors together with some of the processes contributing to the extinction is shown in Fig 3.22.

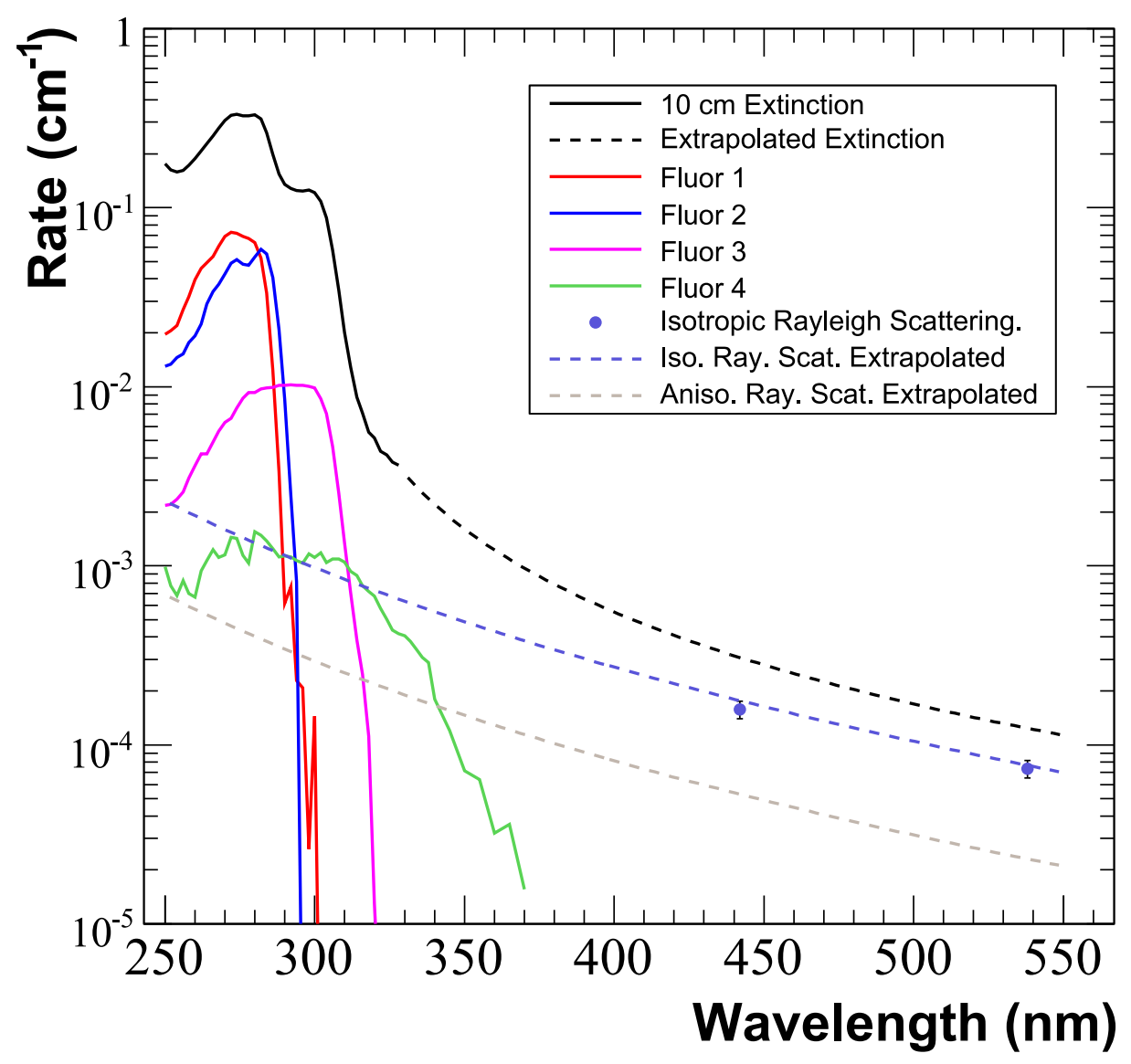

Figure 3.22: Extinction rate as a function of wavelength in the Marcol 7 mineral oil and some of the processes contributing to it. Figure from Ref. [87]

\subsubsection{Simulation of Photomultiplier tube Response and Digital Acquisition}

The final step in the simulation is generating the PMT response of the GEANT3 photoelectrons that reach the PMT. After that the information is passed through a FORTRAN-based code which replicates the passage of data through the DAQ electronics. This ensures that the final MC output is structurally the same as the data. 
The PMT response is simulated by smearing the time and charge accumulated for each photon hit to account for effects like pre-pulsing, late pulsing, and individual PMT behavior, as obtained from the PMT studies mention in $\S 3.5$ and also using the data collected from the laser flask calibration described in $§ 3.7$. A time offset $\Delta t_{\text {off }}$ corresponding to the particular channel is also added.

Digitization of the output of the PMT response simulation is achieved by creating a triangular anode pulse according to the charge drawn for the PE. This pulse is then used to create a set of charge and time quad outputs by simulating the effects of MiniBooNE DAQ as seen in §3.6.1.

\subsection{Event Reconstruction}

Different events (such as electrons, muons, protons, etc.) have different topologies, and consequently different event reconstructions must be applied. Since the event identity is not known a priori, each event is normally reconstructed under different hypotheses, after which particle identification (PID) cuts may be applied, depending on the particular analysis strategy. This section follows Ref. [105].

A NCE scattering event in MiniBooNE results in an outgoing nucleon with nuclear recoil. For the purpose of the reconstruction we ignore the nuclear recoil and reconstruct the outgoing nucleon. The reconstruction applied here is under the assumption that the outgoing nucleon is a proton, while neutrons - if ejected - are only seen through their secondary interaction with a proton.

Protons have a different light emission profile as compared to other particles reconstructed in MiniBooNE (electrons, muons, or pions). Being heavier, protons mostly emit isotropic scintillation light and only emit Cherenkov light above $350 \mathrm{MeV}$, their Cherenkov threshold in the MiniBooNE medium. The NCFitter reconstruction package utilizes both the scintillation and the Cherenkov light to reconstruct the outgoing nucleons. Each of the 1,280 PMTs in 
the main region stores the following information:

- if the PMT registered a hit;

- if the PMT was hit, the measured charge of the hit;

- if the PMT was hit, the measured time of the hit;

Using the above information the reconstruction algorithm reconstructs the particle track. A reconstructed track in MiniBooNE is a vector $\mathbf{X}=\left(\mathbf{x}_{\mathbf{0}}, \mathbf{y}_{\mathbf{0}}, \mathbf{z}_{\mathbf{0}}, \mathbf{t}_{\mathbf{0}}, \theta_{\mathbf{0}}, \phi_{\mathbf{0}}, \mathbf{E}_{\mathbf{0}}\right)$, where $x_{0}, y_{0}, z_{0}$ is the event vertex in the coordinate system of the detector, $t_{0}$ is the event time, $\theta_{0}$ and $\phi_{0}$ define the event direction, and $E_{0}$ is the kinetic energy.

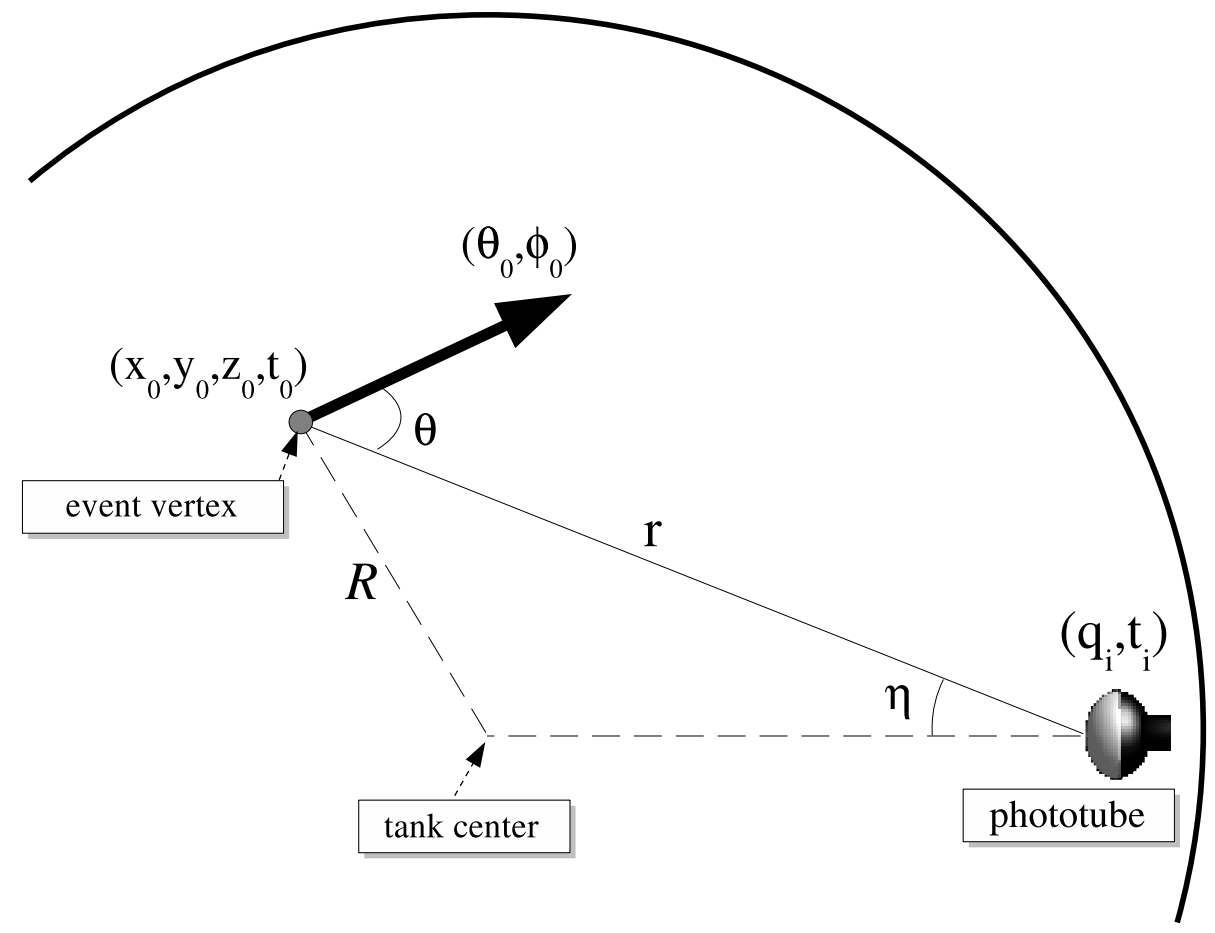

Figure 3.23: Reconstruction geometry.

To reconstruct any event MiniBooNE employs a method of charge and time likelihood maximization. Assuming that the PMTs behave independently, for a given set of PMT 
measurements and track parameters $\mathbf{X}$, the event likelihood is defined as:

$$
\mathcal{L}(\mathbf{X})=\prod_{\mathbf{i}, \text { nohits }} f_{q}(0, \mathbf{X}) \prod_{\mathbf{i}, \mathbf{h i t}} f_{q}\left(q_{i}, \mathbf{X}\right) f_{t}\left(t_{i}, \mathbf{X}\right)
$$

where products are taken over unhit and hit PMTs and

- $q_{i}$ and $t_{i}$ are the measured charge and time at the $i^{\text {th }}$ PMT,

- $f_{q}\left(q_{i}, \mathbf{X}\right)$ is the probability distribution function (PDF) for the measured charge on the $i^{\text {th }} \mathrm{PMT}$, given $\mathbf{X}$, evaluated at $q_{i}$ and

- $f_{t}\left(t_{i}, \mathbf{X}\right)$ is the PDF for the measured time given $\mathbf{X}$, evaluated at $t_{i}$.

The $\mathbf{X}$ dependence of the PDFs can be expressed using the predicted charge $\left(\mu_{i}\right)$ for each PMT:

$$
\begin{aligned}
& f_{q}\left(q_{i}, \mathbf{X}\right)=f_{q}\left(q_{i}, \mu_{i}(\mathbf{X})\right) \\
& f_{t}\left(t_{i}, \mathbf{X}\right)=f_{t}\left(t_{i}, \mu_{i}(\mathbf{X}), E\right),
\end{aligned}
$$

where an additional energy dependence is associated with the time likelihood. As we explain later, the particle's path-length depends on its energy, which in turn affects directly its timing characteristics.

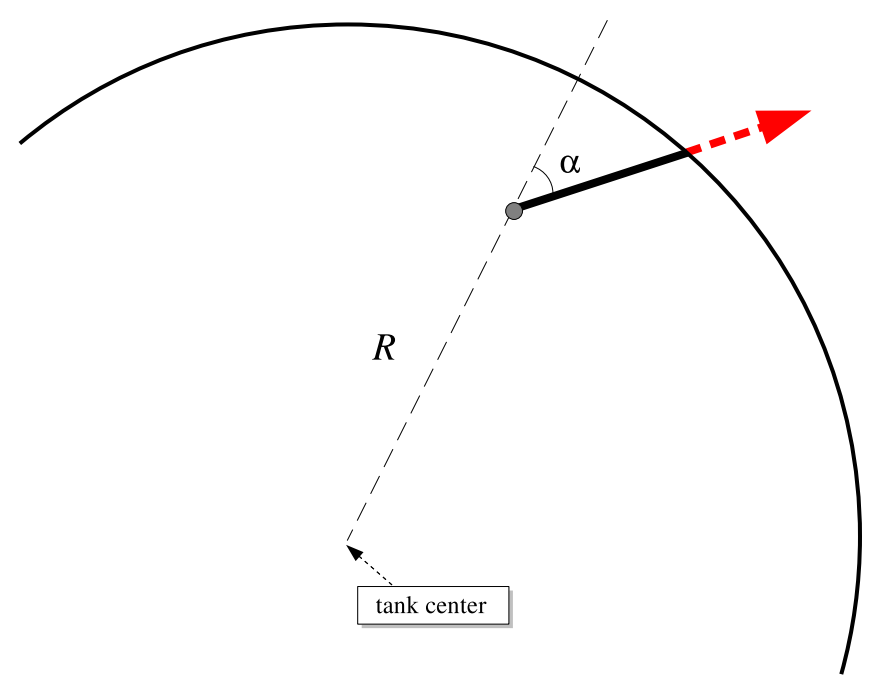

Figure 3.24: Geometry of a long outgoing event. 
The predicted charge as a function of the track parameters, $\mu_{i}(\mathbf{X})$, is for both light components, scintillation and Cherenkov light, as follows:

$$
\begin{aligned}
& \mu_{s c i}(\mathbf{X})=\epsilon \phi_{s c i}(E) \frac{\exp \left(-r / \lambda_{s c i}(R)\right)}{r^{2}} f(\cos \eta) F_{s c i}(E, \cos \theta, R) \operatorname{Corr}(E, \cos \alpha) \\
& \mu_{c e r}(\mathbf{X})=\epsilon \phi_{c e r}(E) \frac{\exp \left(-r / \lambda_{c e r}\right)}{r^{2}} f(\cos \eta) F_{c e r}(E, \cos \theta, R) \operatorname{Corr}(E, \cos \alpha)
\end{aligned}
$$

where the subscripts sci and cer stand for scintillation and Cherenkov, respectively, and:

- $\epsilon$ is the quantum efficiency, i.e., the probability that a photon is detected by a PMT; $\epsilon$ is different for old and new PMTs in MiniBooNE.

- $\phi$ is the light flux - the total number of photons per solid angle created in an event.

- $\lambda$ is the effective attenuation length; for scintillation light this parameter also depends on the radial shell of the event vertex, $R$, which models relatively well other effects besides attenuation, such as scattering, absorption and reemission, etc.

- $f(\cos \eta)$ accounts for the angular acceptance of the PMTs, where $\eta$ is the angle of the incident light with respect to the PMT normal.

- $F(E, \cos \theta, R)$ is the angular emission profile with dependence on energy, $E, \theta$ (the angle between the particle's direction and the line from the PMT to the vertex), and radial shell $R$.

- $\operatorname{Corr}(E, \cos \alpha)$ is a correction function for outgoing events where an energetic proton may leave the tank before losing all its energy and $\alpha$ being the angle between the event direction and the line from center of the tank - see Fig. 3.24.

For a clear picture of the geometric angles refer to Fig. 3.23 and Fig. 3.24.

The charge likelihood $f_{q}(q>0, \mu)$ is used by all reconstruction algorithms, and was obtained from the MiniBooNE laser calibration data described in §3.7.1. The time likelihood, 
$f_{t}\left(t_{i}, \mu_{i}(\mathbf{X}), \mathbf{E}\right)$, is calculated as:

$$
f_{t}\left(t_{c o r r}, \mathbf{X}, \mathbf{E}\right)=\frac{\mu_{s c i}}{\mu_{s c i}+\mu_{c e r}} f_{t}^{s c i}\left(t_{c o r r}, \mu_{s c i}, E\right)+\frac{\mu_{c e r}}{\mu_{c e r}+\mu_{s c i}} f_{t}^{c e r}\left(t_{c o r r}, \mu_{s c i}, E\right),
$$

with contributions from both scintillation and Cherenkov light, as explained below. The event time $\mathrm{t}$ was corrected to $t_{\text {corr }}$ to adjust for the time of photon propagation from the vertex to the PMT using:

$$
t_{c o r r, i}=t_{i}-t_{0}-\frac{r_{i}}{c_{n}}
$$

where $t_{i}$ is the PMT hit time, $t_{0}$ is the event time, $r_{i}$ is the distance to the PMT, and $c_{n}=19.50 \mathrm{~cm} / \mathrm{ns}$ represents the effective speed of light in the detector medium.

A Gaussian distribution is used to represent the Cherenkov light component,

$$
f_{t}^{c e r}\left(t_{c o r r}, \mu_{c e r}, E\right)=\frac{1}{\sqrt{2 \pi} \sigma\left(E, \mu_{c e r}\right)} \exp \left[-\frac{\left(t_{c o r r}-t_{0}\left(E, \mu_{c e r}\right)\right)^{2}}{2 \sigma^{2}\left(E, \mu_{c e r}\right)}\right]
$$

while a slightly more complicated expression is used the model the scintillation part, namely:

$$
\begin{aligned}
f_{t}^{s c i}\left(t_{c o r r}, \mu_{s c i}, E\right)= & (1-B) \frac{1}{2 \tau_{1}} \exp \left(\frac{\sigma^{2}}{2 \tau_{1}^{2}}-\frac{t_{c o r r}-t_{0}}{\tau_{1}}\right) \operatorname{Erfc}\left[\frac{\sigma}{\sqrt{2} \tau_{1}}-\frac{t_{c o r r}-t_{0}}{\sigma}\right] \\
& +B \frac{1}{2 \tau_{2}} \exp \left(\frac{\sigma^{2}}{2 \tau_{2}^{2}}-\frac{t_{c o r r}-t_{0}}{\tau_{2}}\right) \operatorname{Erfc}\left[\frac{\sigma}{\sqrt{2} \tau_{2}}-\frac{t_{c o r r}-t_{0}}{\sigma}\right]
\end{aligned}
$$

where Erfc is the error function complement,

$$
\operatorname{Erfc}(x)=1-\operatorname{Erf}(x) \quad \text { with } \quad \operatorname{Erf}(x)=\frac{2}{\sqrt{\pi}} \int_{0}^{x} e^{-t^{2}} d t
$$

The expression for the scintillation light is nothing else but the result of the convolution of a Gaussian (representing the PMT response) with two exponentials (of time constants $\tau_{1}$ and $\tau_{2}$ ) representing the exponential time response of the scintillator. As for Cherenkov light, the widths and the offsets, $\sigma$ and $t_{0}$, respectively, as well as the time "constants" $\tau_{1}$ and $\tau_{2}$, are actually functions of the predicted charge and event energy. For example, for 
a given energy, the widths of the Cherenkov distributions become narrower with increasing amount of charge, whereas for the same amount of light the distributions become wider with increasing energy - as the particle path-length increases.

For details on reconstruction methods used in MiniBooNE, see Ref. [106]; for the NCE reconstruction in particular and the development of the NCFitter see Ref. [105]. 


\section{Chapter 4}

\section{Analysis}

In this chapter we present the analysis of the antineutrino nucleus NCE scattering data. After

defining our signal, we define the selection cuts and describe the background subtraction. We then describe the procedure employed for unfolding the data to extract the cross section. We also present the error estimation method. Using the extracted antineutrino cross section we take a ratio of the antineutrino nucleus NCE cross section to the neutrino nucleus NCE cross section along with the associated errors. Finally we extract, the axial mass from the antineutrino NCE sample using a $\chi^{2}$ minimization method.

The work presented in this chapter was done as a part of this dissertation research unless otherwise stated.

\subsection{Cross section and Flux Integrated Differential Cross Section}

Before we embark on our stated aim to measure the antineutrino nucleus NCE scattering differential cross section, we clarify some basic terms.

Whenever one set of particles, $N^{s}$ (the source), is impinged upon another set of particles, $N^{t a r}$ (the target), then the cross section $(\sigma)$ is a measure of the probability that a source 
particle will hit or interact with a target particle. In the present experimental setup, the number of source particles $(\bar{\nu})$ is designated as flux $(\Phi)$ and is measured as a function of velocity or energy, and $\sigma$ is measured in terms of the momentum transferred squared $\left(Q^{2}\right)$ to the nucleon by the antineutrino. Finally, since measurements can only be made in terms of certain values of $Q^{2}$, we introduce the differential cross section. The flux is also integrated over the neutrino energy spectrum, as the incoming antineutrino energy cannot be reconstructed. The resulting flux-integrated differential cross section in terms of $Q^{2}$ is

$$
\frac{d \sigma}{d Q^{2}}=\frac{N^{i n t} /\left(\frac{d Q^{2}}{d T^{i n t}} \Delta T^{i n t}\right)}{N^{t a r} N^{P O T} \int \Phi_{\bar{\nu}} d E_{\bar{\nu}}}
$$

where $N^{i n t}$ is the number of interactions; $d Q^{2} / d T^{i n t}$ is the momentum transferred per interaction, $\Delta T^{i n t}$ is the bin width of the interaction spectrum, and $\int \Phi_{\bar{\nu}} d E_{\bar{\nu}}$ is the total integrated flux - a particle flux integrated over time is also called fluence.

\subsection{Signal definition}

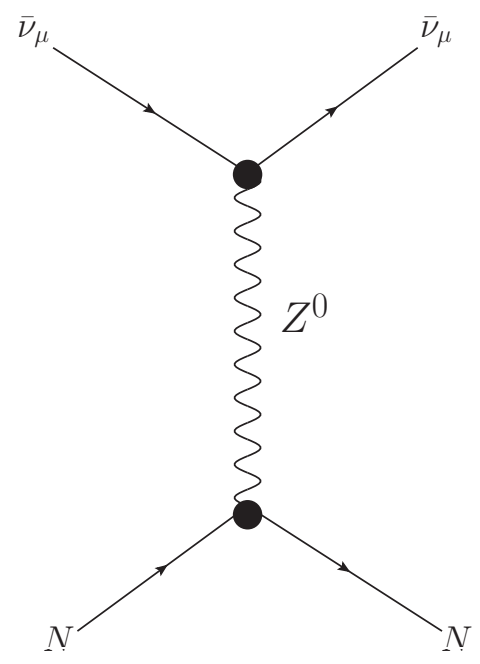

Figure 4.1: Antineutrino nucleon NCE interaction.

In this analysis, our aim is to measure the neutral current elastic scattering of antineutrinos off nucleons, which happens via an exchange of a $Z^{0}$ boson. There is no flavor change and 
the particles remain in the initial states:

$$
\bar{\nu}+N \rightarrow \bar{\nu}+N
$$

Complications to the measurement of the above signal arise due to the fact that MiniBooNE has a nuclear target $\left(\mathrm{CH}_{2}\right)$. The target nucleon can be any one of the 2 protons in the $\mathrm{H}$ atom, 6 protons in the $\mathrm{C}$ atom, or 6 neutrons in the $\mathrm{C}$ atom. In case of the nucleons in the carbon atom, a nucleon, after interacting with the antineutrino, may undergo additional interactions within the nucleus. These interactions are referred to as final state interactions (FSI). MiniBooNE can only detect neutrons if they undergo FSI that result in a proton emission. The final state interactions are not discernible by the detector and we have an "observable" scattering which has to be defined more broadly than shown above. However it should be pointed out that since an "observable" scattering is exactly what the detector sees, it is practically more useful.

To sum up, the the observed signal for the antineutrino neutral current elastic cross section, an antineutrino interacting with a nucleon is defined to include:

- an antineutrino interacting with a proton and the proton is knocked out of the nucleus;

- an antineutrino interacts with a proton and the proton undergoes FSI, resulting in multiple nucleons exiting the nucleus; the average energy of the antineutrino beam $(\sim 0.6 \mathrm{GeV})$ is lower than the threshold for inelastic scattering where the target nucleon is broken up.

- an antineutrino interacts with a neutron and the neutron undergoes FSI possibly knocking a proton out of the nucleus or another nucleus. Both cases result in multiple proton emission. 


\subsection{Analysis Cuts}

NCE events constitute about $18 \%$ of the total events in MiniBooNE. In order to isolate a sample of NCE events we perform a series of analysis cuts. These are various conditions on the experimental variables, like PMT charge, time or reconstructed energy, which differentiates the NCE events from other events in the MiniBooNE sample. These analysis cuts are based on the physics processes and Monte Carlo studies. The development of the selection cuts was done as a part of this dissertation research. We describe below each cut in detail.

\section{- Only 1 subevent.}

A subevent is a cluster of at least 10 tank PMT hits with no more than 10 ns between any two consecutive hits. In order to be classified as subevents of the same event, the subevents must occur within the same DAQ window $(19.2 \mu \mathrm{s})$. For example, a CCQE interaction has two subevents:

$$
\begin{aligned}
& 1^{s t} \text { subevent: } \nu_{\mu}+n \rightarrow \mu^{-}+p \\
& 2^{n d} \text { subevent: } \mu^{-} \rightarrow e^{-}+\bar{\nu}_{e}+\nu_{\mu}
\end{aligned}
$$

The first subevent is associated with the primary neutrino interaction where a muon and proton is produced. The second subevent is associated with the decay of the muon. Fig. 4.2 shows the PMT hit time distribution for a typical CCQE event. 


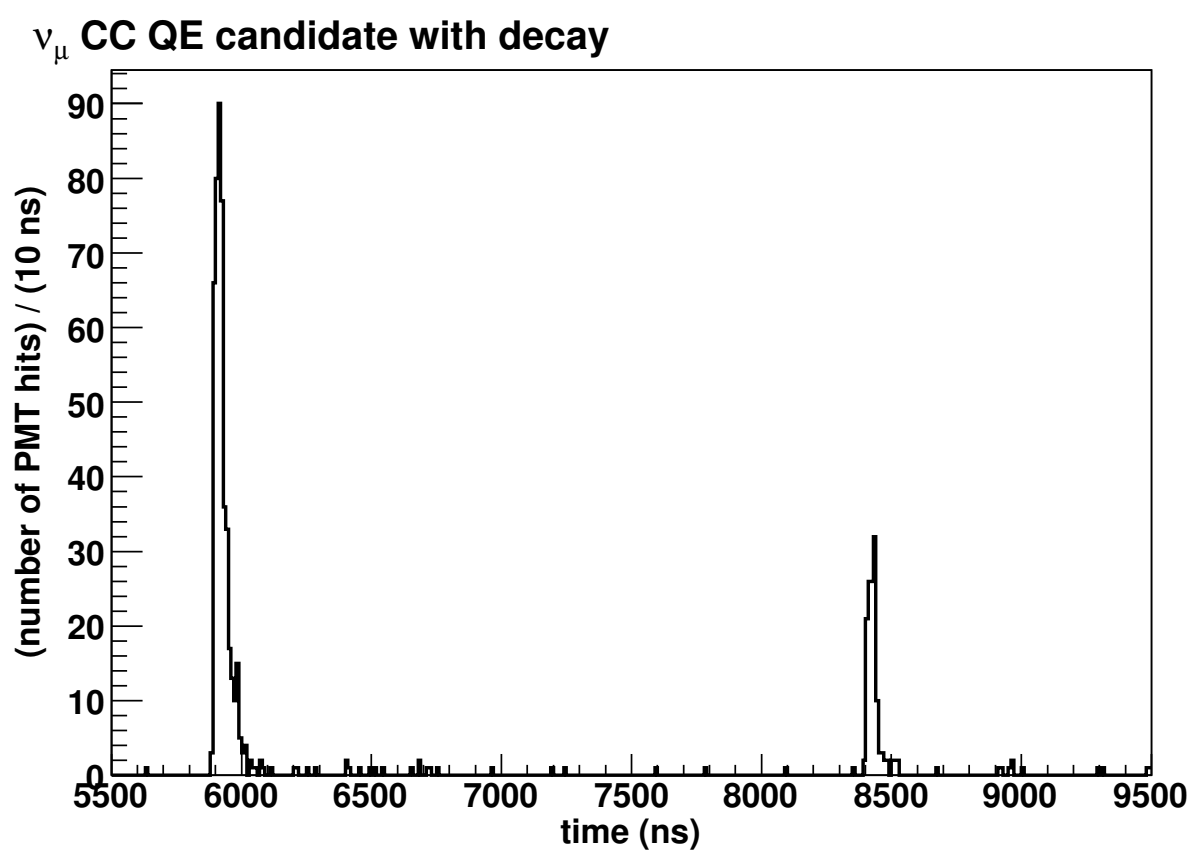

Figure 4.2: A part of beam spill having a CCQE candidate event. The first cluster of PMT hits is from the muon in the primary neutrino interaction, and the secund is due the delayed decay of the muon.From Ref [93]

A typical NCE interaction has only one subevent associated with the primary neutrino interaction.

\section{- Veto hits $<6$.}

This cut excludes events which are entering or exiting the detector and register activity in the veto region. Cosmic rays and neutrino interactions in the material surrounding the detector account for entering events. The veto cuts removes $99.99 \%$ of the cosmic ray background $\mathrm{A}$ CCQE interaction in which the muon exits the detector before decaying can be a background for this analysis. These events also trigger veto activity and are excluded by this cut. 


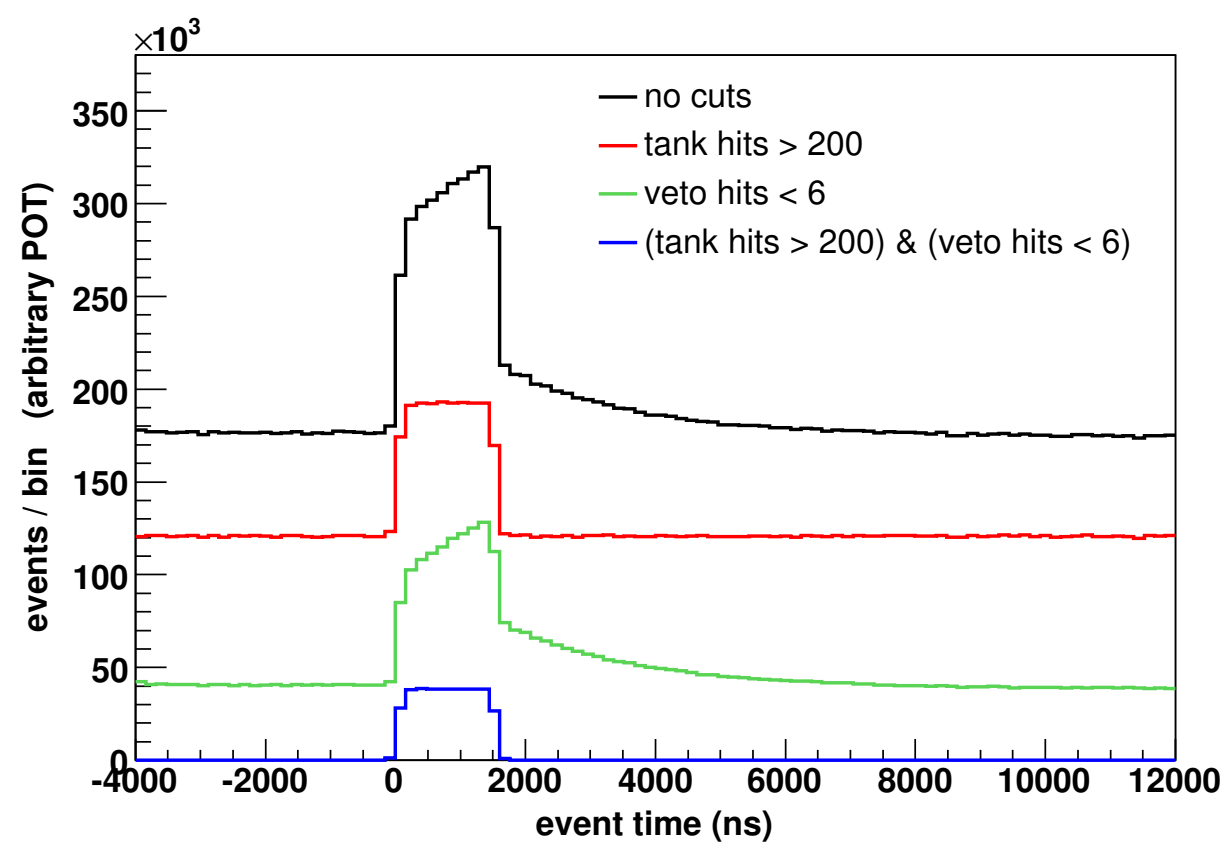

Figure 4.3: The above plot shows the distribution o subevent times after various cuts. The veto hits $<6$ (red to green) cut removes much of the beam-off background. The event window has been shifted to start from zero while making this plot. From Ref [93]

\section{- Tank hits $>12$.}

This cut ensures a minimum number of tank PMT hits so that the event is reconstructable. The NCFitter reconstructs 7 parameters of each event: 3 defining the position, 2 direction parameters, and 1 parameter each for time and energy. At low energies, when protons are below the Cherenkov threshold, only the event position and time can be reconstructed, while the energy can be computed from the total charge and event location. Therefore, to reconstruct the 4 event parameters we require to have at least 3 times as many PMT hits.

\section{- Event in beam window.}

A cut on the reconstructed event time in NCFitter ensures that the event happened within the beam time window, $4400 \mathrm{~ns}<t_{\text {evt }}<6500 \mathrm{~ns}$, and thus the beam-unrelated events are excluded. Here $t_{\text {evt }}=0$ corresponds to start of data taking by the detector which is $\sim 5 \mu$ s before the arrival of the beam. As seen from Fig. 4.3 most of the 
beam-unrelated events are due to Michel electrons from cosmic-ray muon decays.

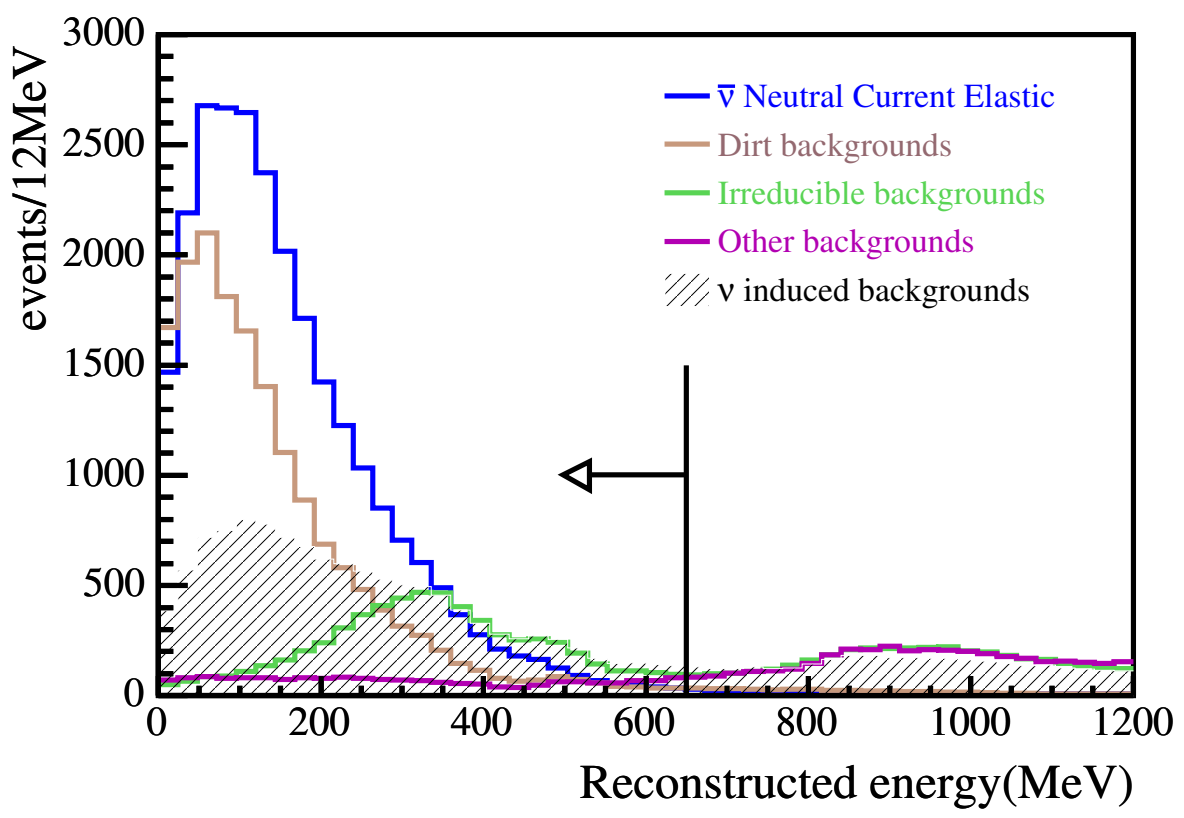

Figure 4.4: The energy cut. Reconstructed nucleon energy distribution for $\bar{\nu}$ NCE signal and various backgrounds. The arrow indicates the part included in the analysis.

\section{- Reconstructed energy $<650 \mathrm{MeV}$.}

As seen in Fig. 4.4 NCE events lie in the low energy region as they produce less light as compared to other neutrino interactions. A cut on the energy while retaining $\mathrm{NCE}$ events, excludes many backgrounds which tend to rise at higher energies. 


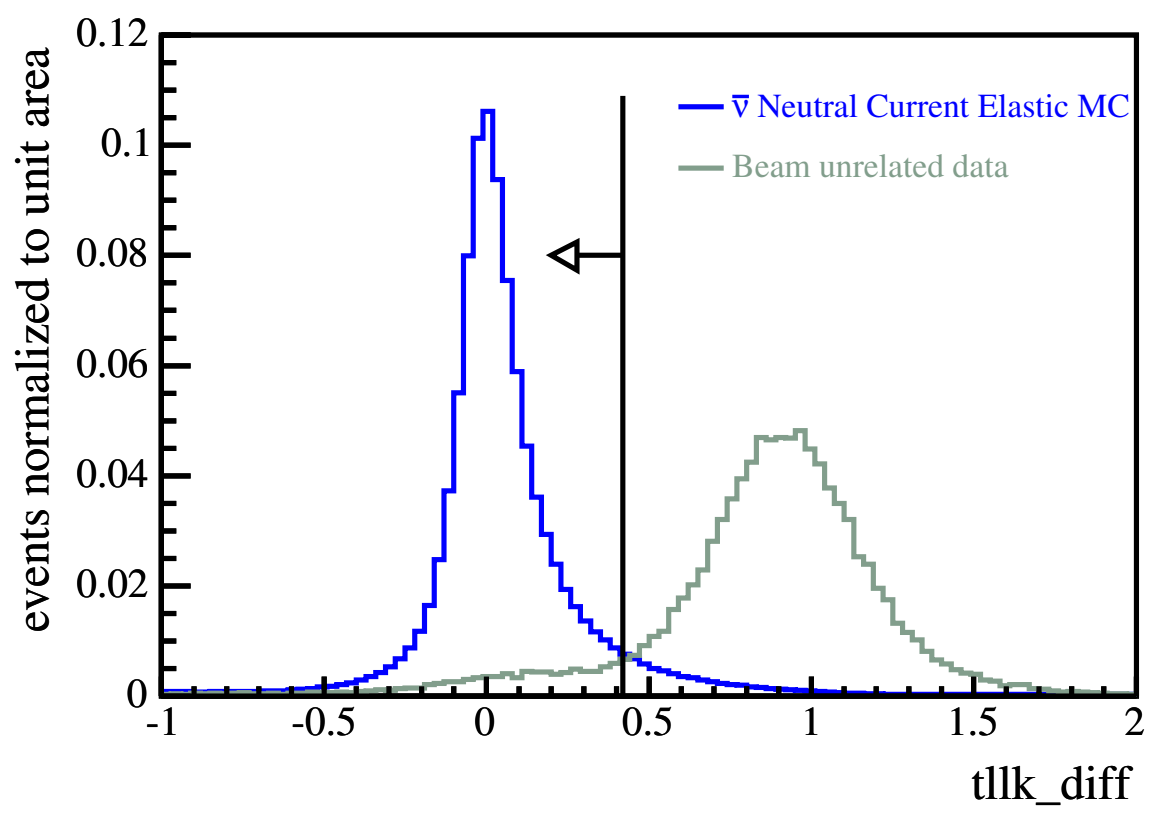

Figure 4.5: The particle identification cut. Time likelihood difference between the proton and electron hypotheses for MC $\bar{\nu} \mathrm{NCE}$ sample. The arrow indicates the part included in the analysis.

\section{- $\ln \left(\mathcal{L}_{e} / \mathcal{L}_{p}\right)<0.42$}

This is a particle identification cut which selects proton-like events while rejecting electron-like events. Fig. 4.5 shows the time likelihood difference between events reconstructed under an electron and a proton hypothesis, for both Monte Carlo NCE scattering events and beam-unrelated backgrounds. The beam-unrelated backgrounds are dominated by electrons, and are significantly reduced as a result of this cut.

\section{- Fiducial volume cut, $\mathrm{R}<5 \mathrm{~m}$.}

The final cut selects a fiducial volume of $R<5 \mathrm{~m}$ where $\mathrm{R}$ is the reconstructed radius in the NCFitter. This cut ensures that the events in the sample are well reconstructed, as the events close to the PMTs cannot be reconstructed reliably. Additionally, this cut also reduces the dirt background, which are neutrino interactions in the dirt surrounding the detector. 


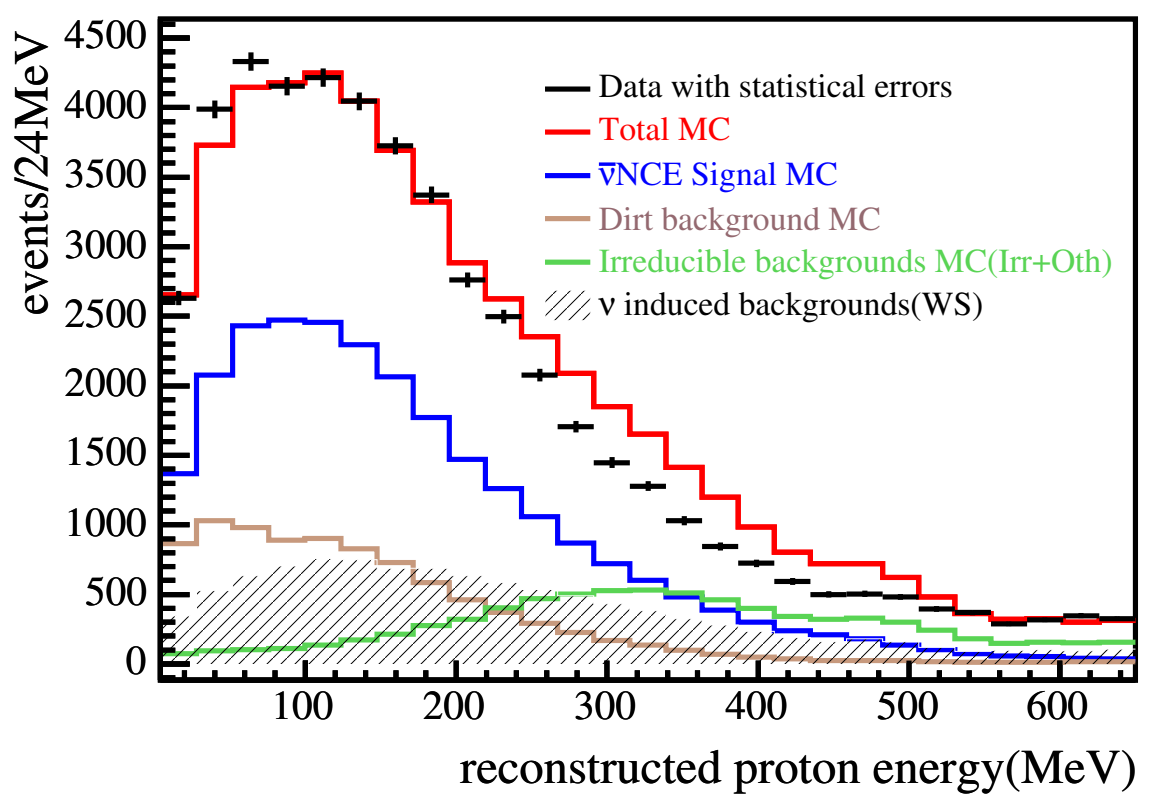

Figure 4.6: Sample composition (after analysis cuts). Reconstructed nucleon energy distribution for data and various backgrounds. Data points are shown with statistical error.

\subsection{Sample composition}

A total of 60,605 events pass the analysis cuts, representing the largest sample of antineutrino NCE scattering events ever collected. The data set corresponds to $10.1 \times 10^{20}$ POT. The cut efficiency is $33 \%$. According to the MC prediction, $48 \%$ of the sample are $\bar{\nu}$ NCE events, and the rest $52 \%$ are backgrounds. The three major backgrounds are dirt events (17\%), irreducible events (14\%) and neutrino-induced events (19\%). Figure 4.6 shows the reconstructed energy spectrum for the data along with the MC predictions for the signal and various backgrounds. There is some disagreement between data and the MC prediction in the low energy region, where the $\mathrm{MC}$ underpredicts the data, and in the intermediate energy region, where the $\mathrm{MC}$ overpredicts the data. We explore a possible reason for this discrepancy in $\S 4.12$.

The dirt events contribute to the background at low energy (below $300 \mathrm{MeV}$ ). These are mostly due to the neutrino interactions in the dirt surrounding the detector, and the 
resulting nucleon passes into the detector without firing enough veto PMTs. The dirt events are difficult to simulate as the material surrounding the detector has not been studied well enough. Hence we use the MiniBooNE data to measure the dirt contributions to the sample.

The irreducible events are so called because they mimic the NCE signal and we have to solely rely on the $\mathrm{MC}$ to estimate them. These are $\mathrm{NC}$ pion events in which the pion is absorbed and only the nucleon is visible, just as in NCE scattering events.

The neutrino-induced events are another major background. These are due to the intrinsic neutrino background in the antineutrino beam. The corresponding antineutrino background in the neutrino mode beam was negligible, due to the lower cross section. MiniBooNE has measured the neutrino background in its antineutrino mode [107] which is used by this analysis to constrain the neutrino induced background.

In the next section we describe the procedure of estimating the various backgrounds in the sample.

\subsection{Estimation of Backgrounds}

In this section we estimate the various backgrounds, namely the dirt background, the $\mathrm{NC} \pi$ irreducible background, and the neutrino induced wrong sign (WS) background.

\subsubsection{Dirt Background}

As mentioned earlier, dirt events in the detector result from neutrino interactions with the material surrounding the detector. The products of theses interactions (neutrons in the neutrino mode and protons in the antineutrino mode) penetrate the detector without firing enough veto PMTs to be vetoed and hence contaminate our sample.

The measurement of the dirt background was a part of this dissertation research.

The dirt background is estimated using the MiniBooNE data. The dirt events have distinct kinematics as compared to in-tank interactions: they are preferentially reconstructed 
upstream $(\mathrm{Z}<0 \mathrm{~m})$ and in the outer regions of the detector with relatively lower energies. The dirt energy spectrum is measured by performing a template fit of the dirt-enriched samples of MC to the MiniBooNE data in three variables, namely the reconstructed event $Z$ coordinate, the radial position, $R$, and energy.

\subsubsection{Generation of Dirt-Enriched Samples}

To measure the dirt background, three additional samples of events with an increased dirt event fraction are generated. This is done for three variables: the $\mathrm{R}$ distribution, the $\mathrm{Z}$ distribution and T (kinetic energy) distribution. They have the following precuts:

- Only 1 Subevent,

- Veto hits $<6$,

- Tank hits $>12$,

- Event in beam window, and

- $\ln \left(\mathcal{L}_{e} / \mathcal{L}_{p}\right)<0.42$.

These are the same selection criteria as for the NCE scattering sample with the exception of the fiducial volume and event energy cuts. In addition, the following cuts are applied on individual samples:

Sample Purpose of sample Cuts: Precuts+

\begin{tabular}{lc}
\hline \hline \multirow{2}{*}{ NCE } & NCE sample (dirt-reduced) \\
& $\mathrm{R}<4.2 \mathrm{~m}$ for $\mathrm{E}<200 \mathrm{MeV} \&$ \\
$\mathrm{R}<5 \mathrm{~m}$ for $\mathrm{E}>200 \mathrm{MeV}$
\end{tabular}

Dirt_Z Fit dirt with Z (dirt-enhanced) $\quad 3.8 \mathrm{~m}<\mathrm{R}<5.2 \mathrm{~m}$

Dirt_R Fit dirt with R (dirt-enhanced) $\quad \mathrm{Z}<0$

Dirt_E Fit dirt with energy (dirt-enhanced) $3.8 \mathrm{~m}<\mathrm{R}<5.2 \mathrm{~m} \& \mathrm{Z}<0$

Table 4.1: Sample names, purpose and cuts to create a dirt-enriched sample. 


\subsubsection{Dirt Measurement from the Z Distribution}

In the measurement of dirt using the $\mathrm{Z}$ distribution we make a total $\mathrm{MC}$ distribution histogram which is the sum of the in-tank MC and the dirt MC histograms. We also have the NCE data for this distribution. We vary the dirt MC and the in-tank MC to find the best fit to the data. We do this in different energy bins (10 bins of $61 \mathrm{MeV}$ each) between $40 \mathrm{MeV}$ and $650 \mathrm{MeV}$. The plots in Fig. 4.7 below show the $\mathrm{Z}$ distribution dirt estimation. Note that the "after fit" total MC better describes the data as compared to the "before fit" total MC. There is not many dirt events in energy bins greater than $350 \mathrm{MeV}$. 


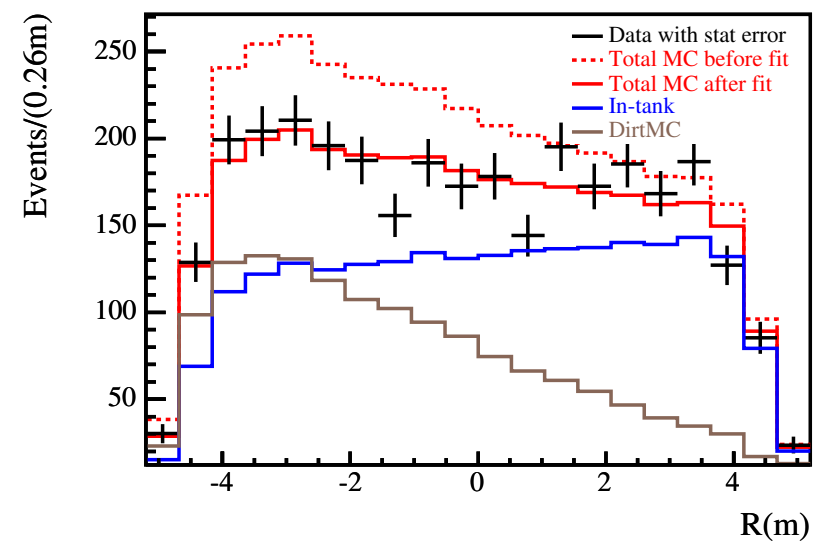

(a)

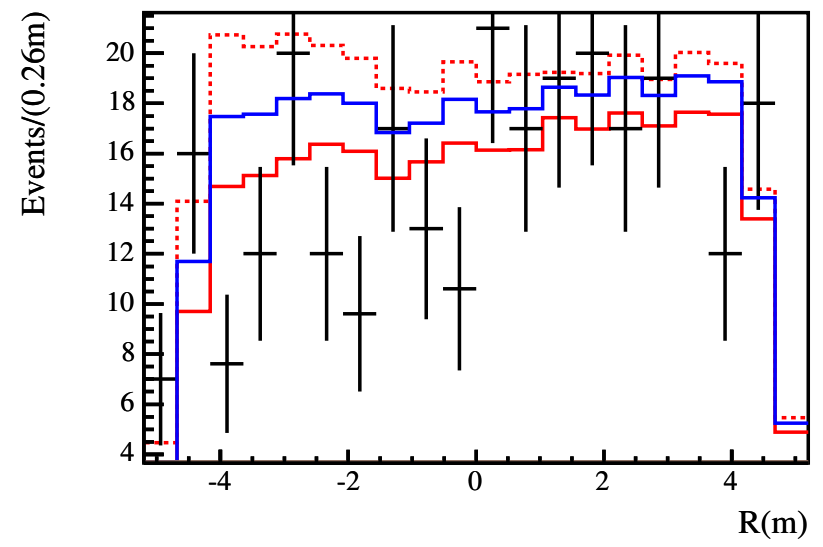

(c)

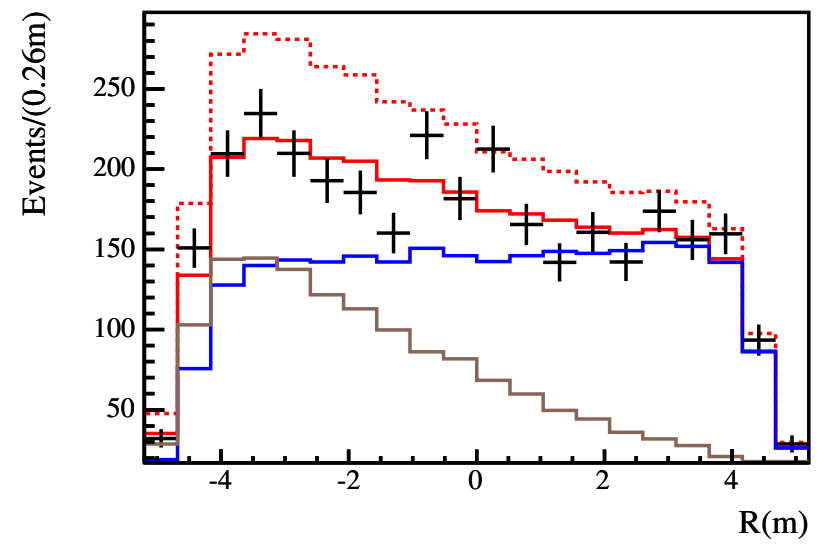

(b)

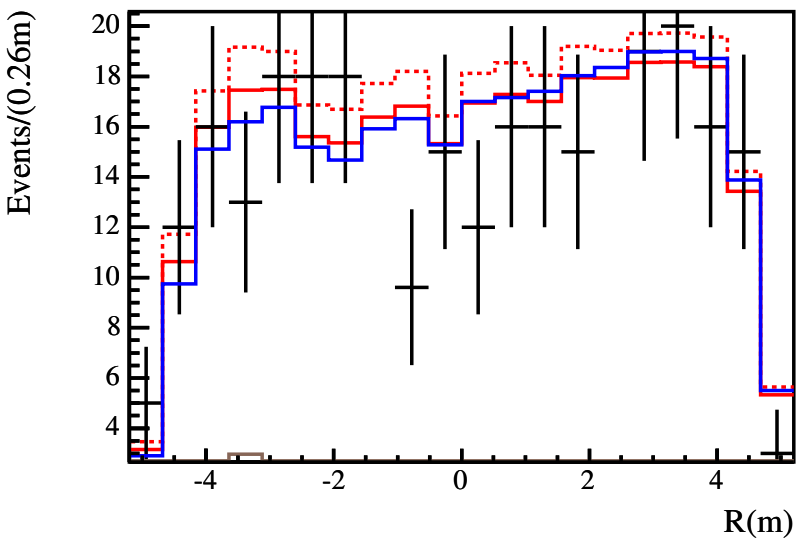

(d)

Figure 4.7: Dirt estimation using $\mathrm{Z}$ distribution. (a) and (b) show the first two energy bins (40-101 MeV and 101-162 MeV) of the 10 energy bins in $\mathrm{Z}$ where the dirt is estimated. Data(black) is shown with statistical errors. Dotted red shows the total (signal +dirt) before fit and solid red shows total MC after fit. Panels (c) and (d) show the last two energy bins, $528-589 \mathrm{MeV}$ and $598-650 \mathrm{MeV}$ bins of the 10 bins. The error bars are relatively large here due to the low statistics.

We obtain a correction function from the best fits in each energy bin. The dirt energy correction function we obtain is shown in Fig. 4.8. The error shown is the error returned by the MINUIT fitting package. 


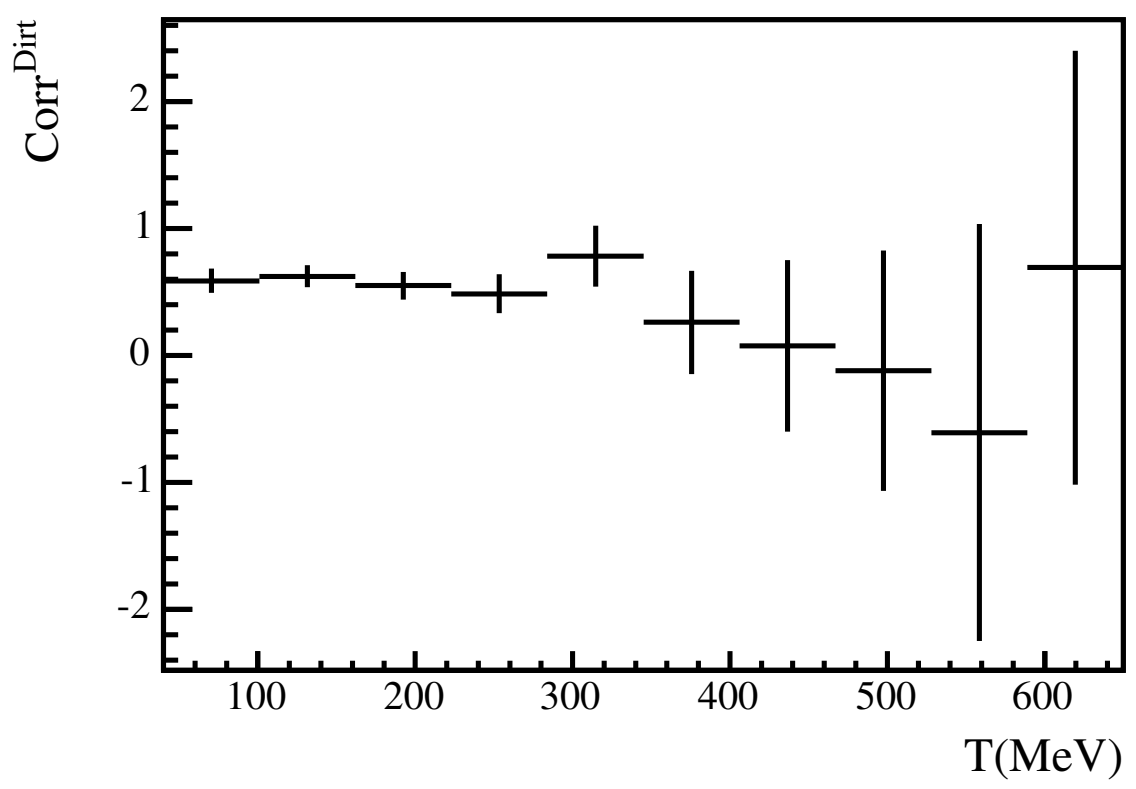

Figure 4.8: Dirt energy correction function from fits in the $\mathrm{Z}$ distribution.

\subsubsection{Dirt Measurement from the $\mathrm{R}$ Distribution}

The dirt measurement using the $\mathrm{R}$ distribution is performed by using the corresponding Dirt_R samples and repeating the above procedure, as shown in [Fig. 4.9]. 


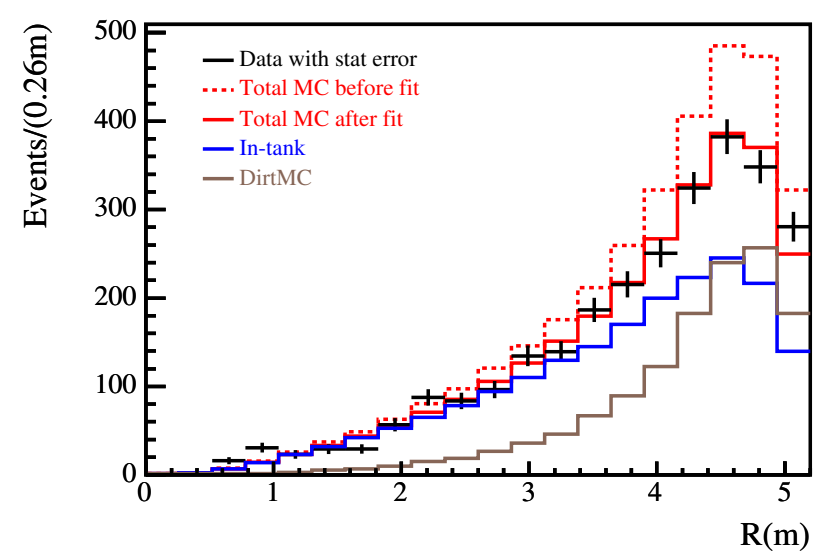

(a)

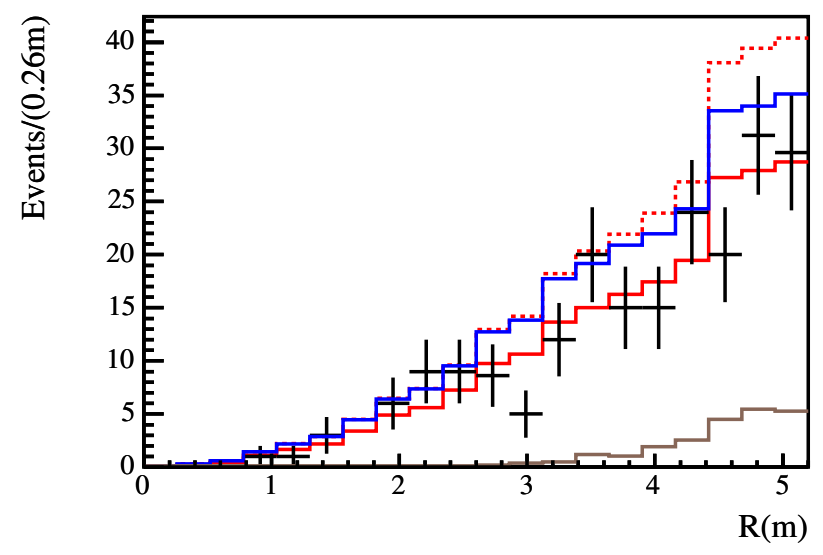

(c)

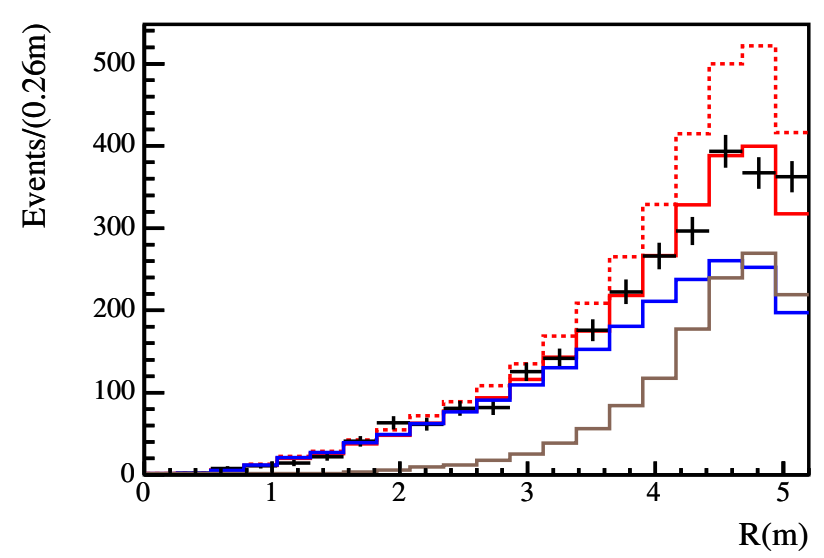

(b)

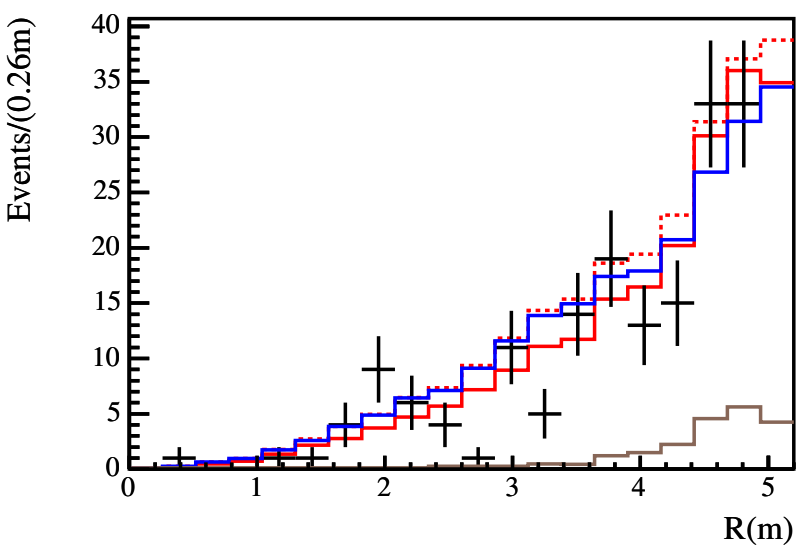

(d)

Figure 4.9: Dirt estimation using the $R$ distribution. Here also panels (a) and (b) show the first two bins ranging from $40-162 \mathrm{MeV}$ where Total MC (solid red) after fit matches better with data. Panels (c) and (d) show the last two energy bins, $528-589 \mathrm{MeV}$ and $598-650 \mathrm{MeV}$ bins, out of the 10 bins where the error bars are relatively large here due to low statistics.

The dirt energy correction function obtained from fitting the $\mathrm{R}$ distribution is shown in Fig. 4.10. 


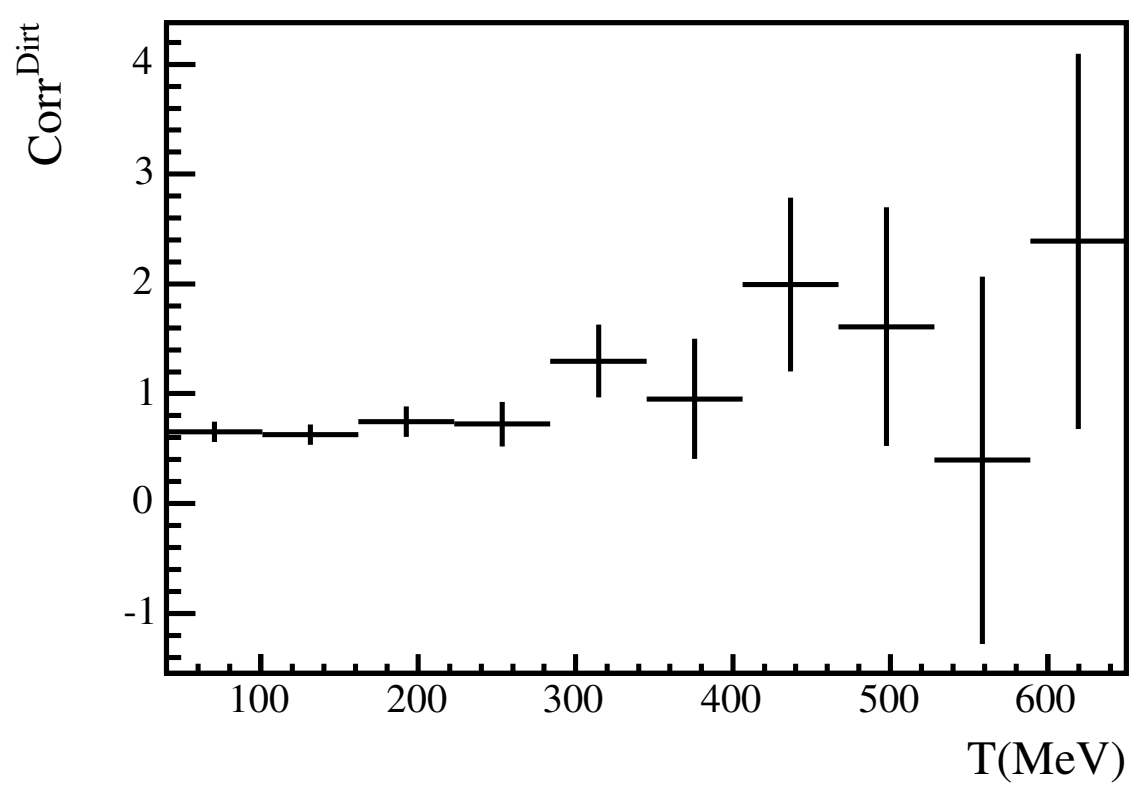

Figure 4.10: Dirt energy correction function from fits in the $\mathrm{R}$ distribution.

\subsubsection{Dirt Measurement from Energy distribution}

In this method dirt is measured using two samples: NCE signal and dirt-enriched sample with Dirt_E cuts. For both samples we have MiniBooNE data, MC prediction for NCE, dirt and in-tank background histograms, as illustrated in [Fig. 4.11]. 

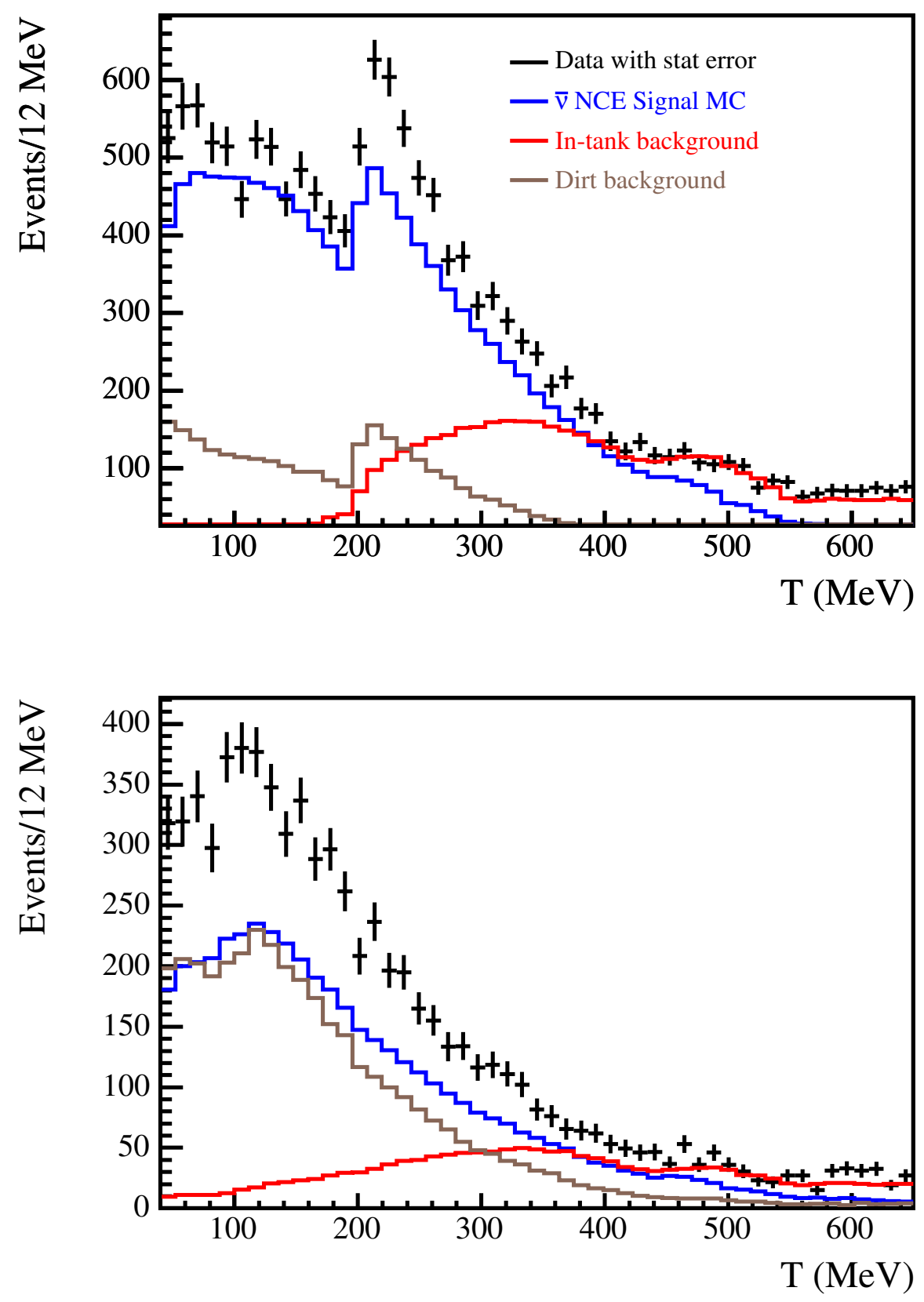

Figure 4.11: Reconstructed energy distribution with NCE cuts (top) and "Dirt_E" cuts (bottom) for data (black) with statistical errors, NCE signal MC (blue), dirt (brown) and intank-backgrounds (green). The spike at $200 \mathrm{MeV}$ for the spectrum on top is due to the energy dependent fiducial volume cut used to make a dirt reduced sample.

We define the following histograms:

$$
\begin{aligned}
& \bar{\nu}=\text { reconstructed energy spectrum for data, } \\
& B=\text { reconstructed energy for MC in-tank backgrounds, }
\end{aligned}
$$




$$
\begin{aligned}
& S=\text { reconstructed energy spectrum for MC NCE signal, and } \\
& D=\text { reconstructed energy spectrum for MC } \operatorname{dirt}
\end{aligned}
$$

with upper indices defined as:

$$
\begin{aligned}
& s=\text { NCE event sample and } \\
& d=\text { Dirt_E event sample }
\end{aligned}
$$

In terms of the definitions introduced we have the following relationships:

$$
\begin{aligned}
& B_{i}^{s}+S_{i}^{s}+D_{i}^{s}=\bar{\nu}_{i}^{s}, \\
& B_{i}^{d}+S_{i}^{d}+D_{i}^{d}=\bar{\nu}_{i}^{d} .
\end{aligned}
$$

Furthermore we define the following fractions in the two samples [Fig. 4.12]:

$$
f_{i}=\frac{D_{i}^{d}}{D_{i}^{s}} \quad \text { and } \quad g_{i}=\frac{S_{i}^{d}}{S_{i}^{s}} .
$$
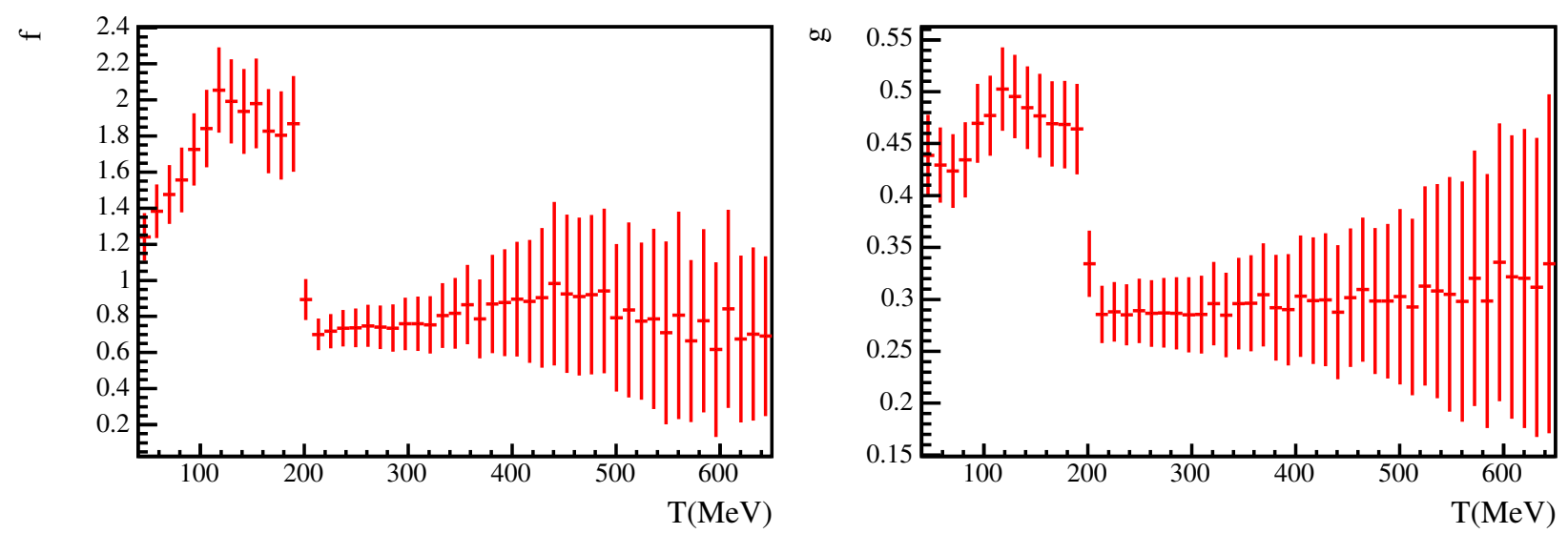

Figure 4.12: fractions $f$ and $g$ as functions of reconstructed energy

Finally one can express $D_{i}^{s}$ (the dirt energy spectrum in the NCE sample) in terms of the above definitions :

$$
D_{i}^{s}=\frac{g_{i}\left(\bar{\nu}_{i}^{s}-B_{i}^{s}\right)-\left(\bar{\nu}_{i}^{d}-B_{i}^{d}\right)}{g_{i}-f_{i}}
$$

The result of this measurement in terms of dirt energy correction function is shown in 
Fig. 4.13.

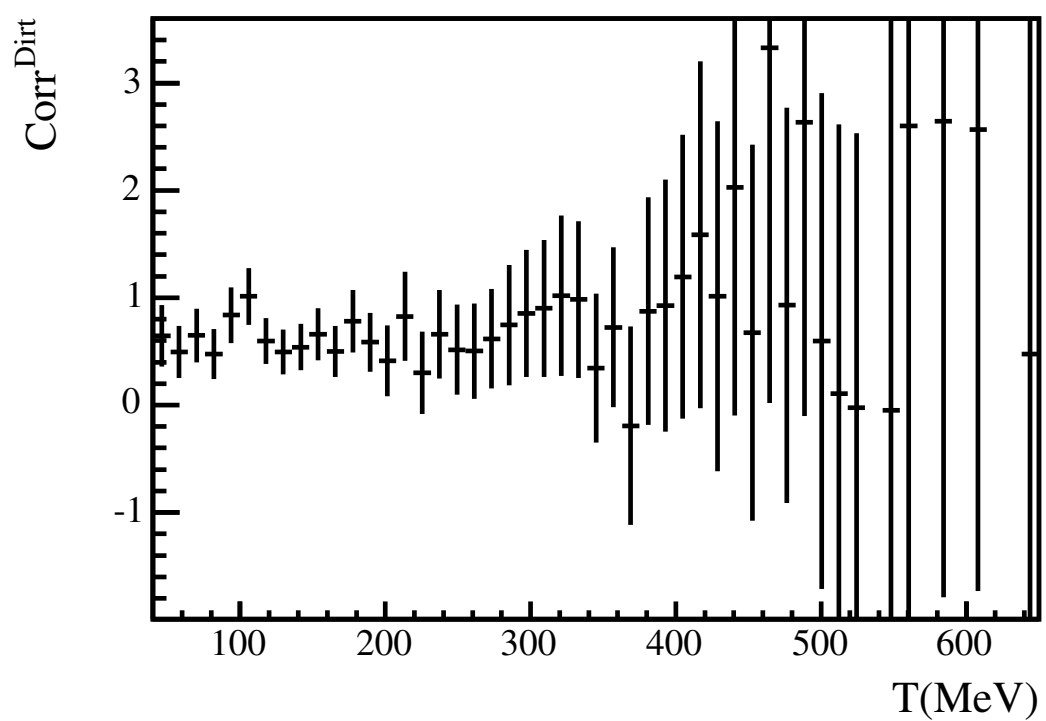

Figure 4.13: Dirt energy correction function from dirt measurement using energy distribution.

\subsubsection{Combined Dirt Correction}

We notice that the errors go up beyond $300 \mathrm{MeV}$ in the dirt energy correction functions obtained by all three methods (Z_dist, R_dist, E_dist). This is because most dirt events have energies below $300 \mathrm{MeV}$ and there are not many dirt events at higher energies to perform the fits. 


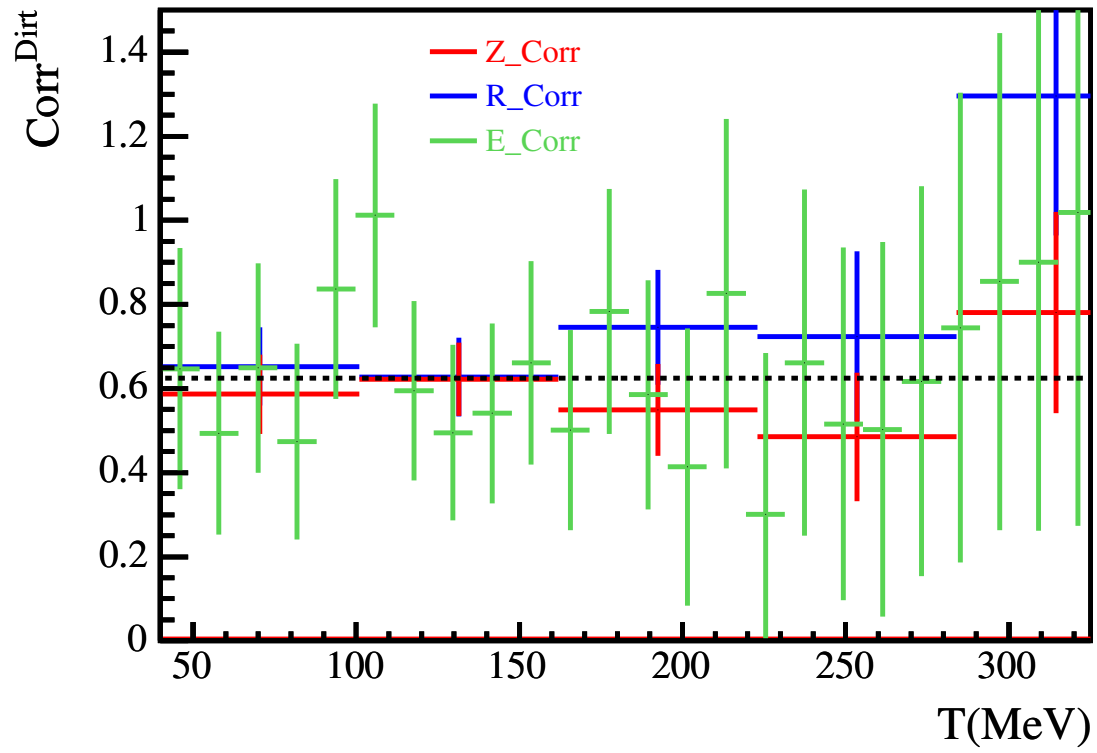

Figure 4.14: Dirt energy correction combined fit using a 0-th order polynomial.

We perform a combined fit on the dirt correction functions obtained by the three methods for the energy range $40 \mathrm{MeV}$ to $325 \mathrm{MeV}$, as shown in Fig. 4.14. The fitted correction function is to a 0 -th order polynomial and yields $0.625 \pm 0.032$, where the error is that returned by the MINUIT fitting package.

\subsubsection{Estimation of Neutrino Induced Background}

Neutrino-induced events in the detector are a major background for the antineutrino NCE scattering cross section measurement. The neutrino contamination in the antineutrino mode is more significant than the antineutrino contamination in the neutrino mode (see Fig. 3.7) due to the leading particle effect: the protons hitting the Be target produce 2 times as many $\pi^{+}$'s as compared to $\pi^{-}$'s, and cross section: at MiniBooNE energies $(\sim 1 \mathrm{GeV})$ the neutrino cross section is 3 times higher than the antineutrino cross section.

In order to estimate the wrong sign (WS) background we refer to MiniBooNE's published measurement of the neutrino component in the antineutrino beam [107] to correct the WS flux and the measured NCE neutrino cross section [60] to correct the WS cross section. 
As discussed in $§ 2.4 .5$, the MiniBooNe flux estimation has inputs from the HARP experiment which studied the proton-Be interactions on a replica MiniBooNE target. However, as seen from Fig. 4.15, there are regions in phase space not covered by the HARP measurements, particularly at small angles where forward peaked pions which are not deflected by the MiniBooNE horn, which are a major source of neutrino contamination at the detector.

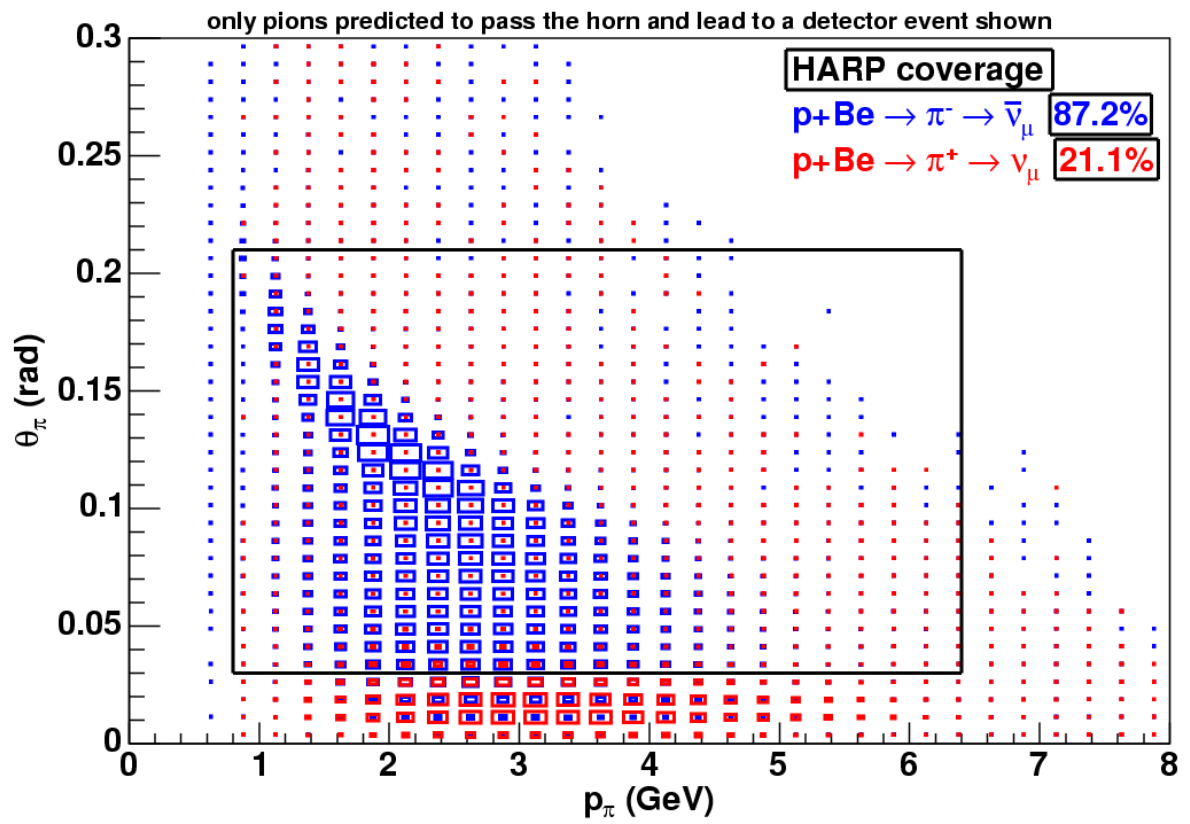

Figure 4.15: Momentum vs pion angle for the MiniBooNE pion production based on MC. The black box encloses the measurements covered by HARP.

MiniBooNE has measured the neutrino component of the antineutrino beam using two independent methods:

1. Using the single $\pi^{+}\left(\mathrm{CC} 1 \pi^{+}\right)$sample: In the antineutrino mode, the $\mathrm{CC} 1 \pi^{+}$events are exclusively due to $\nu_{\mu}$ interactions which can be clearly identified by MiniBooNE. Comparing the data with model prediction for this sample allows to measure the neutrino background.

2. Using the neutrino and antineutrino charged-current quasi elastic (CCQE) sample: The primary muon resulting from a CCQE interaction is more forward peaked in the 
antineutrino induced CCQE interaction as compared to a neutrino induced CCQE interaction. This difference is exploited by performing a simultaneous angular fit to data.

The results of the measurement are summarized in Fig. 4.16 : The $\mathrm{CC} 1 \pi^{+}$analysis indicates that the $\nu_{\mu}$ flux should be scaled by $0.76 \pm 0.11$, while the CCQE angular fits indicate a correction factor of $0.65 \pm 0.23$.

Of the two methods outlined above, method 2 utilizes the antineutrino CCQE scattering interaction. Since the antineutrino NCE scattering interaction shares many of the cross section parameters with the antineutrino CCQE interaction $-M_{A}$ and $\kappa$, for instance - using the WS correction factor from method 2 would instill a model dependence on the present cross section measurement. In a separate study, a third method was used to estimate the neutrino component of the antineutrino beam [108]. This method exploits the fact that stopped negative muons undergo nuclear capture on carbon atom at a rate of $8 \%$ [109], so neutrino induced CCQE events have a significantly lower probability for producing Michel electrons compared to antineutrino CCQE events. The third method suggested that the $\nu_{\mu}$ flux be scaled by $0.81 \pm 0.15$.

For the present analysis, we take the average between the first and the third method and arrive at a correction factor of $0.78 \pm 0.12$ to correct the wrong sign background in the NCE sample. 


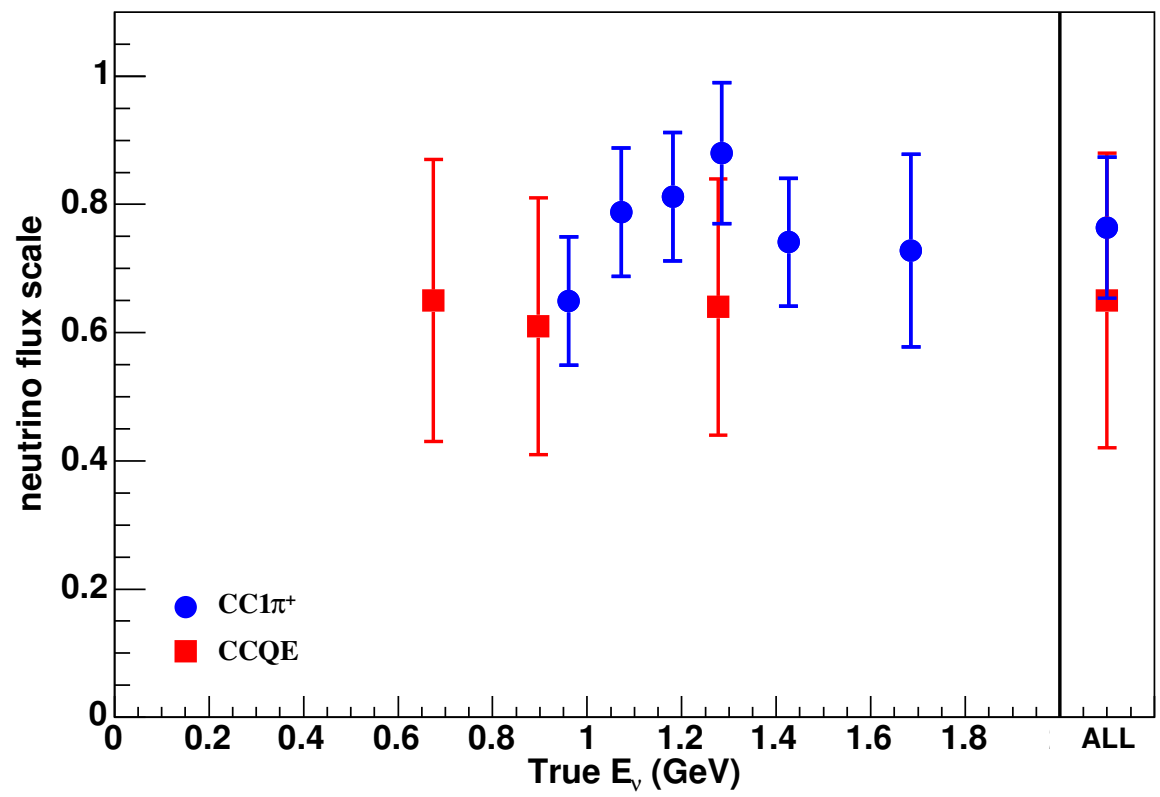

Figure 4.16: Correction factor for neutrino induced events using two independent methods. From Ref [107]

In order to correct the WS cross section we use the neutrino NCE measurement. To summarize the WS component in the antineutrino NCE sample was corrected as:

$$
B_{i}^{W S \_c o r r}=0.78 \times B_{i}^{W S} \times \frac{\nu N C E_{i}^{\text {data }}}{\nu N C E_{i}^{M C}}
$$

where $B_{i}^{W S \_c o r r}$ is the neutrino induced background after WS correction, $B_{i}^{W S}$ is the WS background before correction, and the last term is the correction factor from the neutrino mode cross section measurement. The error associated with the WS estimation is accounted for in the error analysis discussed in $\S 4.7$. 


\subsubsection{The Irreducible Background}

The irreducible background is due to nucleon excitation into a resonant state and subsequent decay of the resonant state to a pion and a nucleon:

$$
\begin{gathered}
\bar{\nu}+p \rightarrow \bar{\nu}+p+\pi^{0}, \\
\bar{\nu}+p \rightarrow \bar{\nu}+n+\pi^{+}, \\
\bar{\nu}+n \rightarrow \bar{\nu}+n+\pi^{0}, \\
\bar{\nu}+n \rightarrow \bar{\nu}+p+\pi^{-} .
\end{gathered}
$$

In addition to the above antineutrino interactions, the irreducible background also includes the corresponding neutrino interactions. The pions may experience an absorption nucleus and as a result the final state of such a neutrino interaction is only nucleons, which is equivalent to the products of the NCE interaction. The MC model is used to estimate the irreducible background.

\subsection{Unfolding}

After the backgrounds have been subtracted the signal needs to be corrected for limited detector resolution, mis-reconstructions, and detector efficiency. This procedure is referred to as unfolding or unsmearing.

The first step in unfolding is using the MC information to migrate the reconstructed energy spectrum to the true energy spectrum, where the true nucleon kinetic energy is the sum of the kinetic energies of all nucleons in final state. In order to do this we employ a $(m \times n)$ migration matrix which correlates the reconstructed and true energy of events that pass the selection cuts:

$$
M_{i j} \quad \text { where } i=1, \ldots, n \text { and } j=1, \ldots, m
$$


where $M_{i j}$ is the number of events with true energy in bin $i$ and reconstructed energy in bin $j$.

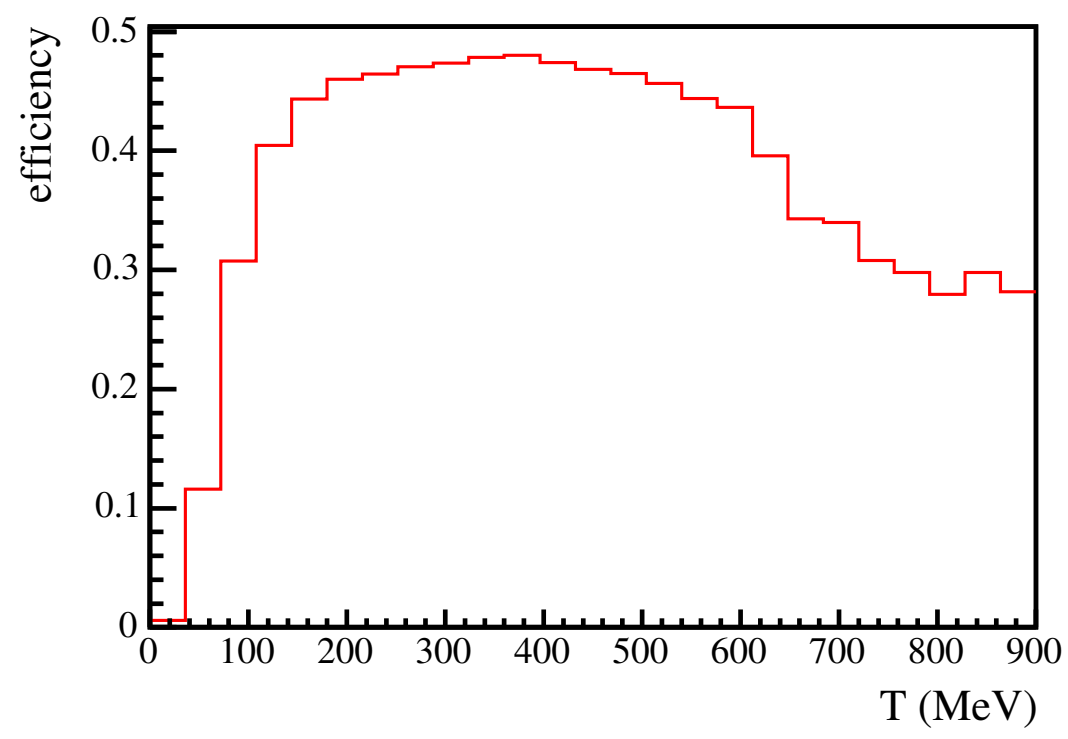

Figure 4.17: Efficiency for $\bar{\nu}$ NCE events with the selection cuts.

The second step is correcting for the limited detector efficiency that distort the original spectrum. This is achieved by defining the efficiency as the ratio of the true energy spectrum before the selection cuts to the same spectrum after cuts:

$$
\epsilon_{i}=\frac{\mu_{i}^{M C, a f t e r \text { cuts }}}{\mu_{i}^{M C, \text { beforecuts }}}
$$

Putting the above two steps together we construct the unfolding matrix $U_{i j}$ :

$$
U_{i j}=\frac{M_{i j}}{\epsilon_{i} \sum_{k=1}^{m} M_{k j}}
$$

where the migration matrix is normalized by dividing each element by the sum of the rows. This method is called the direct matrix method and is based on Bayes' theorem [110]. The 
reconstructed energy spectrum is thus unfolded to the true energy spectrum as follows:

$$
\sigma_{i}^{C V}=\sum_{j} U_{i j}^{C V} D_{j} \frac{S_{j}^{C V}}{S_{j}^{C V}+B_{j}^{C V}}
$$

In the above formula, $\sigma_{i}^{C V}$ is the unfolded energy spectrum. The superscript CV denotes that the unfolding matrix was constructed using the $\mathrm{MC}$ central value (CV) prediction for the migration matrix and efficiency. Here central value refers to the fact that all parameters in the Monte Carlo simulation are set to their nominal values. Similarly we use the CV prediction of signal and backgrounds to subtract the backgrounds from data using the method of signal fraction.

The direct matrix method gives a well-behaved solution but is inherently biased as it depends on the MC prediction of the true and reconstructed spectra. We account for the error introduced due to this bias as a systematic error, which is estimated in $§ 4.8$.

\subsection{Measurement Uncertainties}

Before we measure the final cross section we need to asses the systematic and statistical error associated with the measurement. The systematic errors can be broadly classified into one of the following three types:

A. Flux errors: the uncertainty in the flux is due the combination of the following uncertainties :

- POT error: this error is due to the uncertainty in number of POT delivered to the MiniBooNE target by the Fermilab Booster.

- Beam error: this is the error associated with horn uncertainties and uncertainties in the target that affect the neutrino flux. The uncertainty involves parameters like: skin depth, horn current, nuclear inelastic cross section, nuclear quasi-elastic cross section, pion inelastic cross section, pion quasi-elastic cross section, and pion total cross section. 
- WS error: this error is due to the uncertainty in estimation of neutrino fraction in the antineutrino mode as described in $\$ 4.5 .2$. An uncertainty of $12 \%$ is assigned to this measurement as per Ref. [107].

The next four errors are due to the uncertainties in the meson production in the target namely

- $\mathrm{K}^{0}$ production in the target

- $\mathrm{K}^{+}$production in the target

- $\pi^{+}$production in the target

- $\pi^{-}$production in the target

B. Cross section errors: this class of errors include the uncertainties in the cross section modelling.

- Hadronic model error: the uncertainties in the hadron production model in the MiniBooNE target gives rise to this error.

- $\pi^{0}$ yield error: the uncertainty in the $\pi^{0}$ production in the detector.

- Cross section error: this error accounts for the uncertainties in the cross section parameters shown on table. For the $\bar{\nu}$ NCE cross section measurement, these parameters are allowed to vary only for the background events.

- Dirt error: the uncertainty in the dirt background measurement of $\S 4.5 .1$.

C. Detector errors: this class of errors are due to the limitations of the detector hardware.

- Discriminator threshold error: the error due to the fluctuation of the discriminator threshold value for each PMT.

- Q-T correlation: the error due to the uncertainty in the model of charge-time simulation of the PMTs. 
- Optical model error: this error is due to the uncertainty in the modelling of the optical properties of the detector target medium. The MiniBooNE optical model has 35 parameters which describe among other things, the Cherenkov and scintillation light production, reflections, etc.

The uncertainties associated with the volume, density, and chemical composition of the target and detector materials factor into the uncertainty in the total number of $\operatorname{targets}\left(N^{\operatorname{tar}}\right)$. The density of oil is known to within $0.1 \%\left( \pm 0.001 \mathrm{~g} / \mathrm{cm}^{3}\right)$, the thermal expansion $\left(\sim 10^{-5} / \mathrm{K}\right)$ is negligible [87], and so is the error on Avogardo's number [1] - which is used to calculate the number of target nucleons. For the chemical composition we use $\left(\mathrm{CH}_{2.06}\right)_{n}$ as a model of the oil which differs from the simple model $\left(\mathrm{CH}_{2}\right)_{n}$ by $0.3 \%$. Since the total error associated with the number of target nucleons $(0.3 \%)$ is negligible as compared to the other errors, we do not assign a separate error for it.

Finally, the list of uncertainties also includes

- Statistical error: the statistical fluctuation in the reconstructed energy distribution accounts for the statistical error.

- Unfolding error: the last error is to account for the bias introduced due to the unfolding procedure explained in $\S 4.9$.

Thus we have a total of 16 errors.

\subsection{Multisims and Unisims}

The MC sample used in MiniBooNE depends on various model predictions of flux, cross section, detector effects, etc. As discussed in Chapter 3, the various uncertainties in the model predictions manifest as systematic errors in our measurement. In order to account for these uncertainties, we vary the MC sample with some parameters floated around their best estimates within an allowed region. 
The MC with the best estimation of all its parameters is called the CV. A unisim is a set of MC variation, when only one parameter in a model is varied, and a set of MC is created with the parameter value chosen randomly around its mean with a variance of $1-\sigma$. When several parameters are varied at once, with some parameters correlated, then the resulting set of MC variation is called a multisim.

For each of the errors mentioned in $\S 4.7$ a set of multisim/unisim is created and the uncertainty due to that error is estimated as described below in $\S 4.8$.

\subsection{Error Calculation}

In this section we describe the method used to estimate the various errors, namely the systematic errors, the statistical error, the unfolding error, and finally the total error.

\subsubsection{Systematic Errors}

In the present analysis we treat each MC variation or multisim as a possible MC CV (best estimate of the prediction). We then calculate the migration matrix and efficiency associated with each MC variation and unfold data.

For each $k$-th multisim the unfolded true spectrum is:

$$
\sigma_{i}^{k}=\sum_{j} U_{i j}^{k} D_{j} \frac{S_{j}^{k}}{S_{j}^{k}+B_{j}^{k}}
$$

where $i$ is the bin of true energy, $j$ is the bin of reconstructed energy, $U_{i, j}^{k}$ is the unfolding matrix for the $k$-th multisim, $D$ is the data, $S$ the signal, and $B$ backgrounds. Again the error associated with the unfolding procedure is calculated as a separate systematic error §4.9.3. The error associated with each systematic error is calculated as:

$$
E_{i}^{\sigma}=\sqrt{\frac{1}{N} \sum_{k=1}^{N}\left(\sigma_{i}^{k}-\sigma_{i}^{C V}\right)^{2}},
$$


where $N$ is the number of $\mathrm{MC}$ variations for each multisim.

\subsubsection{Statistical Error}

The statistical error for this measurement is due to the statistical fluctuations in the observable variable- the reconstructed proton energy. The error on each reconstructed energy spectrum for data is $\sqrt{D_{j}}$.

We construct a unisim where each bin of the spectrum has a value randomly chosen from

a Gaussian with a mean value of $D_{j}$ and variance $\sqrt{D_{j}}$. We then propagate this unisim through the unfolding procedure to estimate the final statistical uncertainty in the cross section.

\subsubsection{Unfolding Errors}

The final error we consider is the error due to the bias in the unfolding procedure. As mentioned earlier, the unfolding matrix depends on the $\mathrm{MC}$ prediction that was used to produce it, and is biased. To eliminate the bias we need to unfold it over several iterations, and for each iteration, using the unfolded distribution as the new true energy spectrum for the next iteration. Theoretically, the unfolded distribution should converge after several iterations. However, when unfolding data, certain bins with low statistics do not converge as compared to MC.

Based on MC studies (which were based on 8 times the statistics as compared to the data) we observed that the difference between the unfolded spectrum and the "true" spectrum is less than $1 \%$ after 7 iterations. Hence, we assume that the bias goes away after approximately 7 iterations, and we calculate the bias in unfolding for each $k$-th multisim:

$$
\operatorname{Bias}_{i}^{k}=\sigma_{i}^{k, 7^{t h} i t e r}-\sigma_{i}^{k, 1^{s t} i t e r}
$$

where $i$ is the bin of true energy for the $k$-th MC variation. The bias for the particular 
systematic error (particular multisim/unisim) is calculated as:

$$
\text { Multisim_Bias } s_{i}^{a}=\sqrt{\frac{1}{N_{a}} \sum_{k=1}^{N_{a}}\left(\text { Bias }_{i}^{k}\right)^{2}} \text {, }
$$

where $N_{a}$ is the number of MC variations for the $a^{\text {th }}$ error.

The total unfolding bias for the 15 systematic and statistical multisims (unisims) is

$$
\text { Unfolding_error }_{i}=\sqrt{\frac{1}{15} \sum_{k=1}^{15}\left(\text { Multisim_Bias }_{i}^{k}\right)^{2}} .
$$

\subsubsection{Total Error}

Adding up all the 16 errors (14 systematic errors, 1 statistical error, and the unfolding error) in quadrature gives the total uncertainty in the cross section measurement.

The individual contribution of each error towards the total uncertainty was also estimated. We do this by taking the ratio of the integral of an individual error over the range of true energy spectrum to the total integral of the unfolded true energy spectrum. Table 4.2 lists the individual error contribution thus calculated and also the total error.

\subsection{The $\bar{\nu}$ Neutral Current Elastic Differential Cross-section}

As explained in $\$ 4.1$, the differential cross section is measured in terms of the momentum transfer squared to the nucleon, $Q^{2}$. In MiniBooNE $Q^{2}$ is is the total kinetic energy of the outgoing nucleons in the interaction, assuming the target nucleon is at rest viz.

$$
Q^{2}=2 M_{N} \sum_{i} T_{i}=2 M_{N} T
$$




\begin{tabular}{clr} 
Type of Error & Source of error & value $\%$ \\
\hline \hline \multirow{2}{*}{ Statistical } & & 6.3 \\
\hline & POT & 1.7 \\
& Beam & 2.9 \\
\multirow{2}{*}{ Flux } & $\mathrm{K}^{0}$ production in the target & 0.1 \\
& $\mathrm{~K}^{+}$production in the target & 0.3 \\
& $\pi^{-}$production in the target & 4.7 \\
& $\pi^{+}$production in the target & 0.1 \\
& WS estimation & 3.5 \\
\hline \multirow{2}{*}{ Cross section } & Hadronic interactions & 2.9 \\
& Cross-section (backgrounds) & 3.1 \\
& Dirt estimation & 1.7 \\
\hline \multirow{2}{*}{ Detector } & Discriminator threshold & 1.1 \\
& Charge-time PMT response & 1.8 \\
& Optical Model & 15.0 \\
\hline Unfolding & & 7.3 \\
\hline Total & & 21.0
\end{tabular}

Table 4.2: Individual error contributions to the total antineutrino NCE scattering cross section error.

where $M_{N}$ is the nucleon mass and $\mathrm{T}$ is the sum of the kinetic energies of the final state nucleons (the summation index $i$ goes over the final state particles). Experimentally, $\sum T_{i}$ is proportional to the total visible energy deposited in the PMTs in the detector.

All that is left to do is to calculate the last few ingredients that go into the differential cross section formula, namely:

- Number of target nucleons, $N^{\text {tar }}$

This is calculated as $N^{t a r}=N_{A} \rho_{\text {oil }}\left(4 \pi R^{3} / 3\right)$ where $N_{A}$ is Avogadro's number, $\rho_{\text {oil }}=$ $0.845 \pm 0.001 \mathrm{~g} / \mathrm{cm}^{3}$, and $\mathrm{R}=610.6 \mathrm{~cm}$ (MiniBooNE detector radius). Since the error on $N^{\text {tar }}$ is negligible (0.3\%) as compared to the other errors we do not assign an error on it. See $\S 4.7$.

- Number of protons on target $\left(N_{P O T}\right)$

This corresponds to $10.1 \times 10^{20}$ for the data set. 
- Integrated neutrino flux $\left(\int \Phi_{\bar{\nu}} d E_{\bar{\nu}}\right)$

This is the total integrated neutrino flux for $\bar{\nu}_{\mu}$ and $\bar{\nu}_{e}$

Putting it all together, the flux-integrated differential cross section is given by:

$$
\frac{d \sigma_{i}^{\bar{\nu} N C E}}{d Q^{2}}=\frac{\sigma_{i} /\left(\frac{d Q^{2}}{d T_{N}} \Delta T_{N}\right)}{N^{t a r} N^{P O T} \int \Phi_{\bar{\nu}} d E_{\bar{\nu}}}
$$

where $\sigma_{i}$ is the number of entries in the $i^{\text {th }}$ bin of the unfolded kinetic energy distribution; $d Q^{2} / d T_{N}=2 M_{N}=1.877 \mathrm{GeV}$, and $\Delta T_{N}=0.018 \mathrm{GeV}$ is the bin width of the unfolded kinetic energy distribution.

Figure 4.18 shows the MiniBooNE antineutrino nucleon NCE scattering cross section. The uncertainties include both systematic and statistical errors. Even though the systematic errors are mostly scaling errors, they do show bin by bin variation in case of certain errors. For instance, the optical model has large uncertainties in the low $Q^{2}$ region, while the flux error has large uncertainties in the high $Q^{2}$ region. 


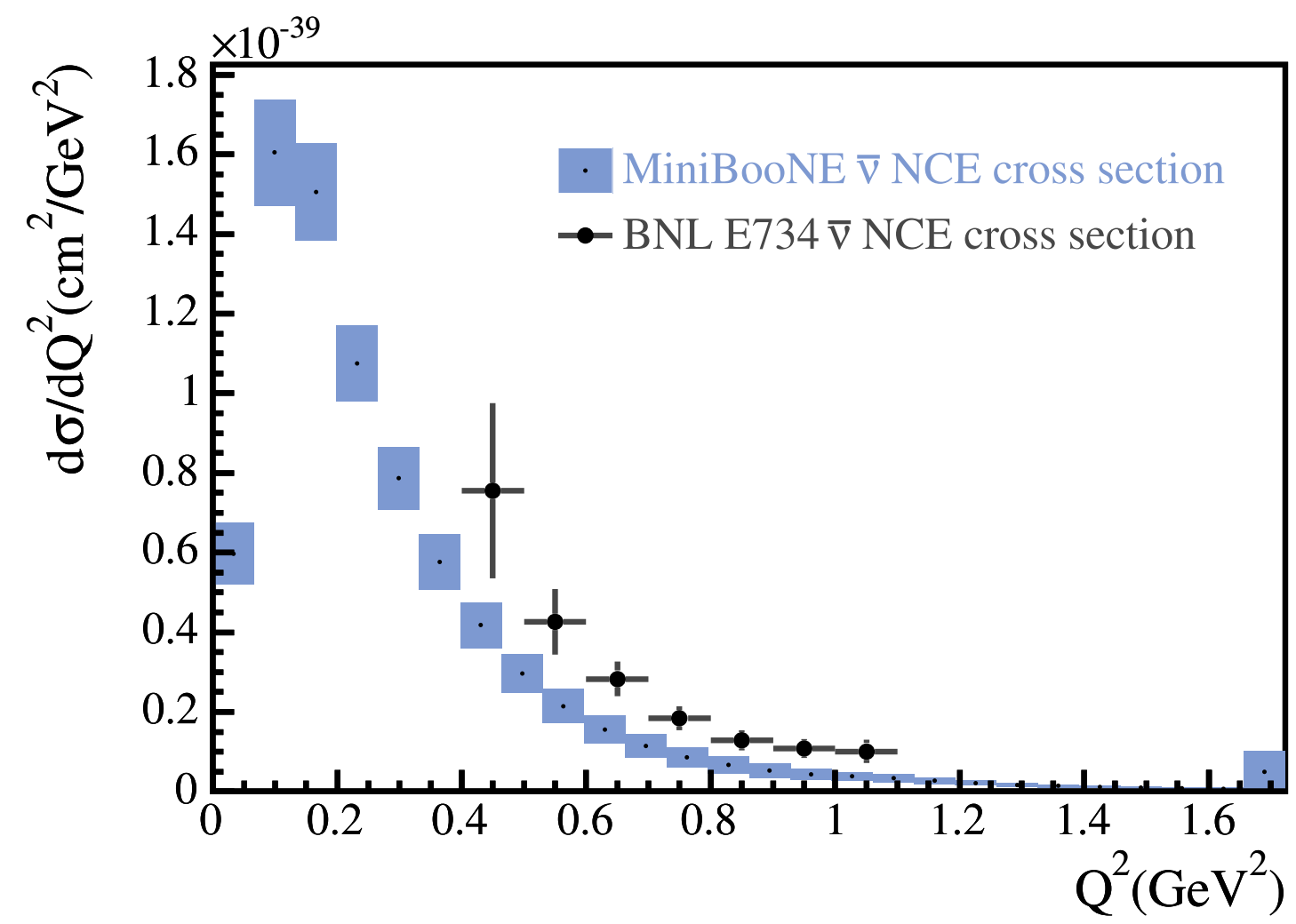

Figure 4.18: MiniBooNE $\bar{\nu} N \rightarrow \bar{\nu} N$ flux-integrated differential cross section on $\mathrm{CH}_{2}$ (grey band) and the BNL E734 $\bar{\nu} p \rightarrow \bar{\nu} p$ flux-averaged differential cross section (brown). The uncertainty includes all errors - systematic and statistical.

Also shown in Fig. 4.18, is the previous measurement of this cross section done by the BNL E734 experiment [62]. The two measurements are not directly comparable, since they represent flux-averaged cross sections with different average neutrino energies. The BNL E734 antineutrino beam had an average energy of about $1.2 \mathrm{GeV}$, as compared to the MiniBooNE antineutrino beam of approximately $0.6 \mathrm{GeV}$. The higher BNL E734 beam energy is the reason for the higher cross section. (Note that the errors in the BNL E734 measurement shown in Fig. 4.18 have been calculated from Tables VIII, IX, and X in Ref. [62].)

The MiniBooNE target is essentially $\mathrm{CH}_{2}$, which implies that the antineutrino NCE scattering cross section includes three different processes:

1. the antineutrino scattering off free protons in the hydrogen atom, 
2. the antineutrino scattering off bound protons in the carbon atom and

3. the antineutrino scattering off bound neutrons in the carbon atom,

Therefore, the MiniBooNE $\bar{\nu} N \rightarrow \bar{\nu} N$ cross section is expressed as:

$$
\frac{d \sigma_{\bar{\nu} N \rightarrow \bar{\nu} N}}{d Q^{2}}=\frac{1}{7} \frac{d \sigma_{\bar{\nu} p \rightarrow \bar{\nu} p, H}}{d Q^{2}}+\frac{3}{7} \frac{d \sigma_{\bar{\nu} p \rightarrow \bar{\nu} p, C}}{d Q^{2}}+\frac{3}{7} \frac{d \sigma_{\bar{\nu} n \rightarrow \bar{\nu} n, C}}{d Q^{2}}
$$

where each individual term is the the cross section per nucleon. 


\subsection{Antineutrino to Neutrino Neutral Current Cross-Section Ratio}

MiniBooNE has already reported the neutrino nucleus neutral current scattering cross section using the world's largest data sample of such events [60]. This unprecedented statistics allowed the measurement of a differential cross section. The antineutrino cross section reported here also represents the largest sample of such events collected to date. Since both measurements were made in the same beamline, with the same detector and using the same analysis software, we expect that many of the systematic errors are common to both. A bin-by-bin ratio of the two cross section measurements would result in cancellation of the common systematic errors. The resulting cross section ratio plot would encompass information from both the neutrino and antineutrino NCE scattering cross section while minimizing the errors. One of the main motivations for measurement of this cross section is to better understand and model the neutrino nucleon interactions. We believe that such a ratio measurement where the errors are carefully accounted for would aid the theoretical physics community to test the various models.

\subsubsection{The Data Set}

The data set for the ratio measurement consists of the entire neutrino mode and antineutrino mode neutral current cross section data. This consists of 94,531 neutrino-nucleon neutral current elastic candidate events which passed the selection cuts corresponding to $6.46 \times 10^{20}$ POT. In the antineutrino mode, a total $10.1 \times 10^{20}$ POT was collected resulting in some 60,605 events that pass the selection cuts. Figure 4.19 shows both the neutrino and the antineutrino cross sections as a function of same $Q^{2}$ bins. 


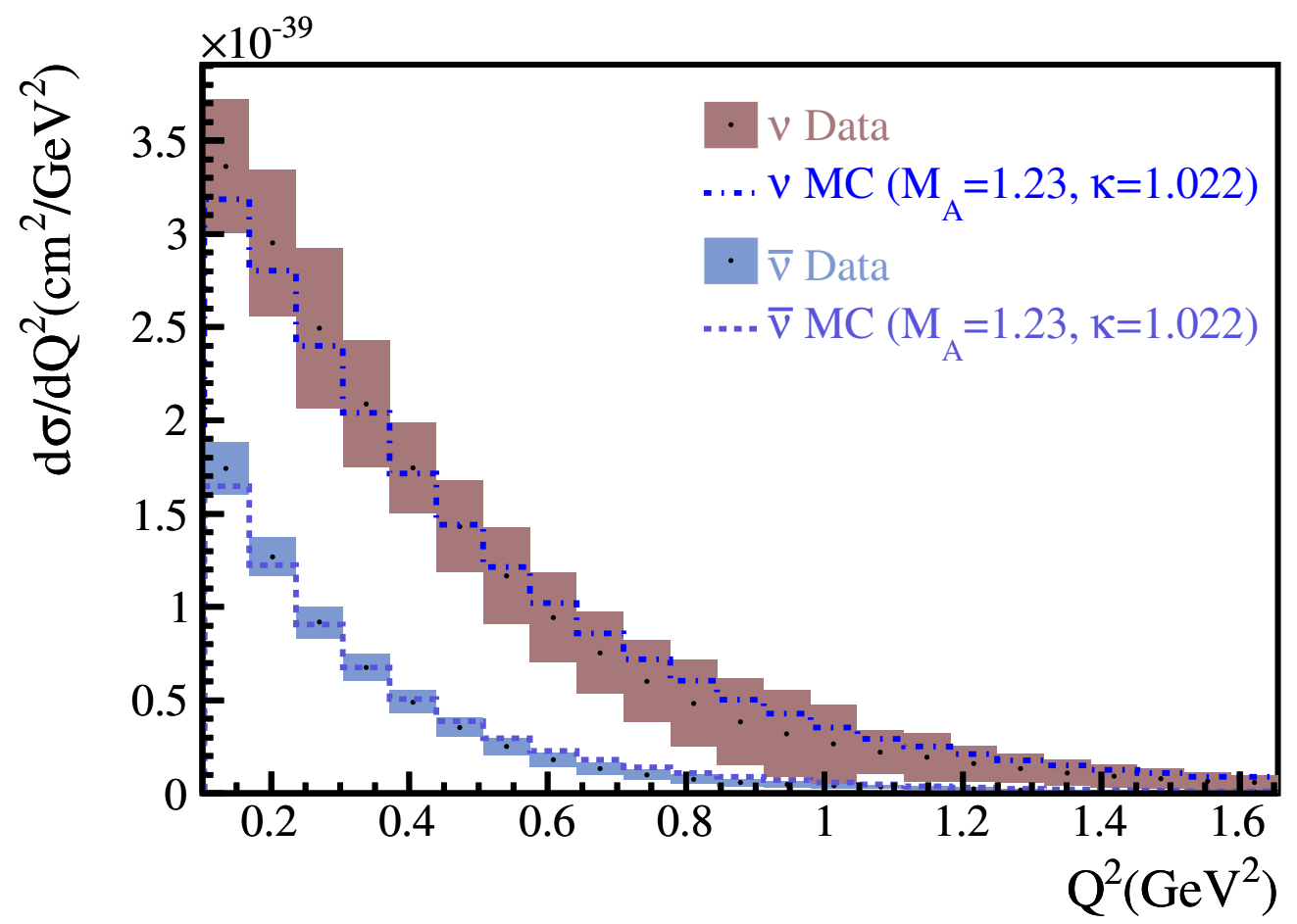

Figure 4.19: A bin-by-bin comparison of the neutrino and antineutrino NCE scattering differential cross sections.

\subsubsection{Error Estimation}

In order to account for the errors in the ratio of antineutrino to neutrino cross section, we divide the errors into two classes, namely correlated errors and uncorrelated errors.

\subsubsection{Correlated errors}

Correlated errors are those errors which are common to both the neutrino and antineutrino modes. These include: the POT error, beam error, $K^{0}$ production error, $K^{ \pm}$production error, $\pi^{ \pm}$production error, hadronic interactions, cross-section, $\pi^{0}$ yield error, discriminator threshold error, charge-time PMT response error, and the optical model error. For a detailed description of the stated errors see $\S 4.7$.

To estimate the error, we employ the same multisim method described in $\S 4.8$. First we obtain the unfolded true energy spectrum for each $k$-th multisim for both the antineutrino 
and neutrino modes:

$$
\sigma_{i}^{k, \bar{\nu}}=\sum_{j} U_{i j}^{k, \bar{\nu}} D_{j}^{\bar{\nu}} \frac{S_{j}^{k, \bar{\nu}}}{S_{j}^{k, \bar{\nu}}+B_{j}^{k, \bar{\nu}}} \quad \text { and } \quad \sigma_{i}^{k, \nu}=\sum_{j} U_{i j}^{k, \nu} D_{j}^{\nu} \frac{S_{j}^{k, \nu}}{S_{j}^{k, \nu}+B_{j}^{k, \nu}}
$$

where the superscripts $\bar{\nu}$ and $\nu$ denote antineutrino and neutrino, $i$ is the bin of true energy, $j$ is the bin of reconstructed energy, $U_{i, j}^{k}$ is the unfolding matrix for the $k$-th multisim, $D$ is the data, $S$ the signal, and $B$ backgrounds.

In the above unfolding procedure, the $k$-th multisim denotes the same variation of $\mathrm{MC}$ parameters in both modes - antineutrino and neutrino. Thus, the unfolded spectrum for the ratio is:

$$
\sigma_{i}^{k, \bar{\nu} / \nu}=\frac{\sigma^{k, \bar{\nu}}}{\sigma_{i}^{k, \nu}}
$$

The CV unfolded spectrum for the ratio is

$$
\sigma_{i}^{C V, \bar{\nu} / \nu}=\frac{\sigma^{C V, \bar{\nu}}}{\sigma_{i}^{C V, \nu}}
$$

where we use the same unfolding procedure as for the multisims, except now it is just for the one "best-estimate" or central value.

For each of the correlated errors, the error matrix is evaluated as:

$$
E_{i, j}^{\bar{\nu} / \nu}=\sqrt{\frac{1}{N} \sum_{k=1}^{N}\left(\sigma_{i}^{k, \bar{\nu} / \nu}-\sigma_{i}^{C V, \bar{\nu} / \nu}\right)^{2}}
$$

where $N$ is the number of MC variations for each multisim.

\subsubsection{Uncorrelated errors}

The uncorrelated errors, which include the dirt estimation error, the unfolding error, and the WS estimation error, do not cancel out in the ratio. In order to estimate the uncorrelated error contribution to the total error in the ratio measurement, first the individual 
uncorrelated error in each mode is estimated:

$$
E_{i}^{\bar{\nu}}=\sqrt{\frac{1}{N} \sum_{k=1}^{N}\left(\sigma_{i}^{k, \bar{\nu}}-\sigma_{i}^{C V, \bar{\nu}}\right)^{2}} \text { and } E_{i}^{\nu}=\sqrt{\frac{1}{N} \sum_{k=1}^{N}\left(\sigma_{i}^{k, \nu}-\sigma_{i}^{C V, \nu}\right)^{2}} \text {. }
$$

Then, we use the formula for error propagation in a ratio where there is no correlation between the individual errors:

$$
E_{i}^{\bar{\nu} / \nu}=\sqrt{\left(\frac{\sigma_{i}^{C V, \bar{\nu}}}{\sigma_{i}^{C V, \nu}}\right)^{2}\left[\left(\frac{E_{i}^{\bar{\nu}}}{\sigma_{i}^{C V, \bar{\nu}}}\right)^{2}+\left(\frac{E_{i}^{\nu}}{\sigma_{i}^{C V, \nu}}\right)^{2}\right]}
$$

The total error on the ratio is evaluated by adding each of the $E_{i}^{\bar{\nu} / \nu}$ s in quadrature. Table 4.3 shows the individual and total error, in the antineutrino mode, the neutrino mode, and the ratio of the two modes.

\begin{tabular}{llrrr} 
Type of Error & Source of error & \multicolumn{3}{c}{ value $\%$} \\
\hline \hline \multirow{2}{*}{ Statistical } & & $\bar{\nu}$ mode & $\nu$ mode & ratio \\
\hline \multirow{3}{*}{ Flux } & POT & 6.3 & 3.1 & 6.9 \\
& Beam & 1.7 & 1.8 & 0.0 \\
& $\mathrm{~K}^{0}$ production in the target & 2.9 & 4.7 & 7.3 \\
& $\mathrm{~K}^{+}$production in the target & 0.1 & 0.1 & 0.1 \\
& $\pi^{-}$production in the target & 4.7 & 0.1 & 0.4 \\
& $\pi^{+}$production in the target & 0.1 & 4.1 & 4.7 \\
& WS estimation & 3.5 & 0.0 & 4.0 \\
\hline \multirow{2}{*}{ Cross section } & Hadronic interactions & 2.9 & 4.2 & 5.9 \\
& Cross-section (backgrounds) & 3.1 & 3.4 & 5.9 \\
& Dirt estimation & 1.7 & 1.7 & 2.3 \\
\hline \multirow{2}{*}{ Detector } & Discriminator threshold & 1.1 & 1.6 & 1.4 \\
& Charge-time PMT response & 1.8 & 2.3 & 2.1 \\
& Optical Model & 15.0 & 15.0 & 6.6 \\
\hline Unfolding & & 7.3 & 8.3 & 15.0 \\
\hline Total & & 21.0 & 25.0 & 25.0
\end{tabular}

Table 4.3: NCE scattering cross section errors for the neutrino mode, the antineutrino mode, and the ratio. 


\subsubsection{Result}

The bin-by-bin ratio of the antineutrino NCE cross section to the neutrino NCE cross section was made and is shown in Fig 4.20. The correlated and uncorrelated errors were estimated and are also shown in the same plot. The ratio measurement cancels out many of the systematic errors. Statistical fluctuations account for the residual error in those systematics which are fully correlated. Also shown in Fig. 4.20 are MC predictions for different values of $M_{A}$ and $\kappa$. We note that the ratio is relatively independent of variations in $M_{A}$ and $\kappa$.

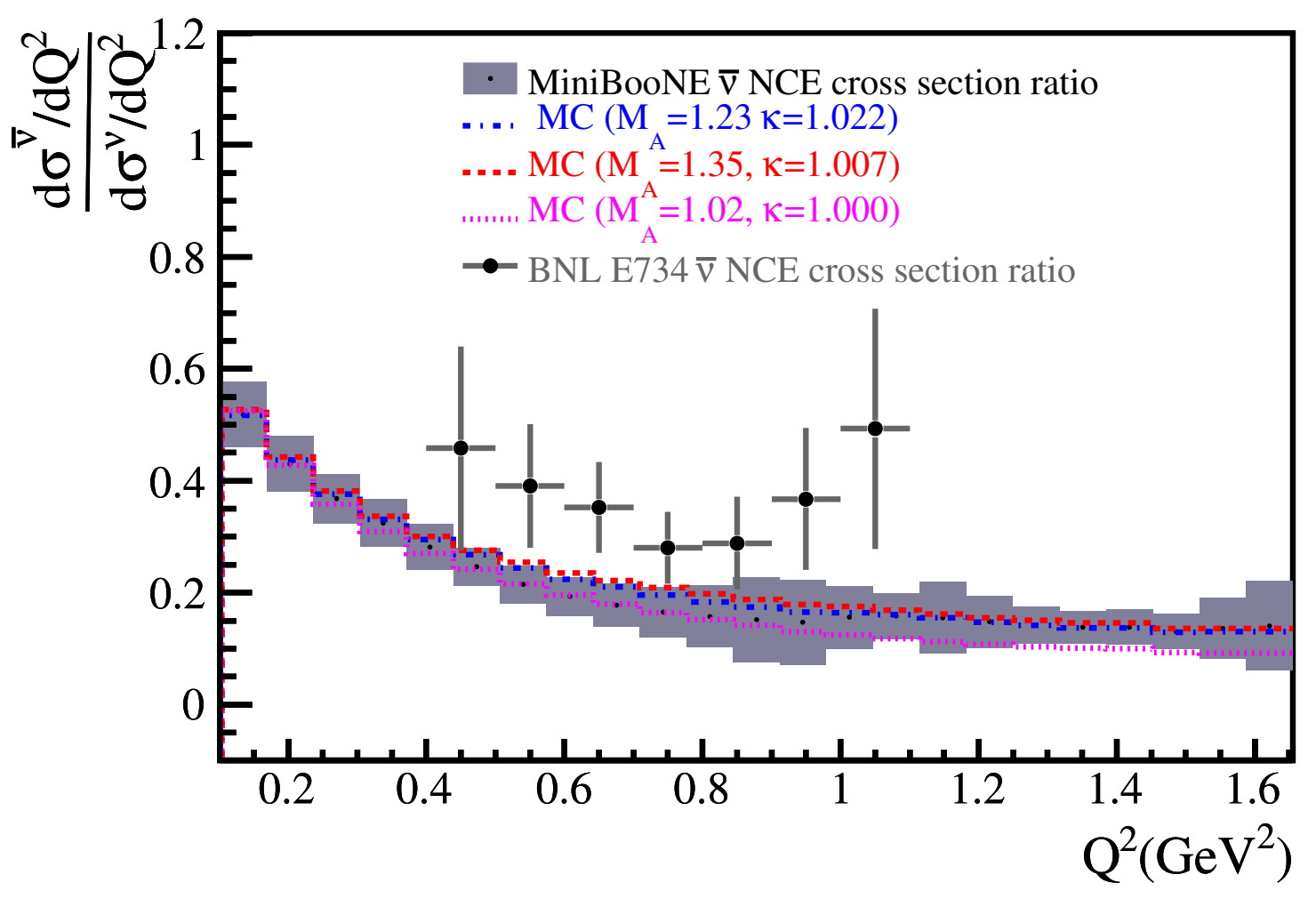

Figure 4.20: Ratio of antineutrino to neutrino NCE scattering cross section in MIniBooNE with total error (grey band). The predicted ratio from MC simulations with different $M_{A}$ and $\kappa$ values is shown superimposed. Also shown is the calculated ratio of antineutrino to neutrino NCE scattering cross section from the BNL E734 experiment (brown) [62] with the errors in the two modes added in quadrature. This overestimates the error in the ratio for the BL E734 measurement as many systematic errors are expected to be correlated and hence cancel.

An antineutrino to neutrino differential cross section ratio plot for the BNL E734 experiment was generated from Fig. 35 from Ref. [62] and overlaid on the MiniBooNE ratio 
measurement in Fig. 4.20. The errors on the BNL E734 experiment are overestimated as the errors in the individual neutrino and antineutrino measurement have been added in quadrature. Many systematic errors would cancel in the case of BNL E734 experiment just as in the MiniBooNE measurement.

We note that the BNL E734 ratio measurement is higher than the MiniBooNE measurement. However, as previously stated, the two experiments are not strictly comparable; the different ratio of the antineutrino to neutrino average beam energies in the BNL E734 and MiniBooNE results in different cross section ratios. For the BNL E734 experiment the average neutrino and antineutrino beam energies were about $1.3 \mathrm{GeV}$ and $1.2 \mathrm{GeV}$, respectively, whereas in MiniBooNE the average neutrino and antineutrino beam energies were approximately $0.8 \mathrm{GeV}$ and $0.6 \mathrm{GeV}$, respectively.

The MiniBooNE antineutrino NCE cross section measurement allows us to make a direct comparison to the neutrino mode NCE cross section measurement made by MiniBooNE, which was done using the same target and beamline, but with the opposite polarity of the magnetic focussing horn. 


\subsection{Axial Vector Mass Measurement}

One of the parameters in the antineutrino-nucleus neutral current cross section is the axial vector mass, $M_{A}$. As discussed in $\S 2.4$, measurements made by deuterium-based bubble chamber experiments have reported a value of $M_{A}=1.026 \pm 0.021 \mathrm{GeV}$. However recent experiments on nuclear targets (such as MiniBooNE with a $\mathrm{CH}_{2}$ target) have reported $M_{A}$ values which are about 20-30\% higher. In the the CCQE channel MiniBooNE reported a value of $M_{A}=1.35 \pm 0.17 \mathrm{GeV}[59]$ and the neutrino mode NCE analysis reported a value of $M_{A}=1.39 \pm 0.11 \mathrm{GeV}[60]$, consistent with that from the CCQE channel.

In this section we attempt a $\chi^{2}$ - fit to the antineutrino neutral-current data, to extract the value of $M_{A}$ which best describes it. In principle, one needs to fit the observed cross section data to the cross section MC prediction for different values of $M_{A}$ in $Q^{2}$ bins. However, we can exploit the relation between the momentum transfer and the nucleon kinetic energy in MiniBooNE NCE interactions, $Q^{2}=2 M_{N} T_{N}$ to do the fitting in bins of reconstructed energy instead of $Q^{2}$. This avoids the unfolding procedure and the associated unfolding error. In order to account for the uncertainties, both systematic and statistical, we also construct an error matrix which is used in the fitting procedure.

For this study, the data sample has the standard NCE cuts and background subtraction procedure as described in $\S 4.3$ and $\S 4.5$, respectively. A reweighting procedure was used to generate antineutrino NCE signal MC with different values of $M_{A}$ and $\kappa$.

Figure 4.21 shows the $\mathrm{MC}$ with different values of $M_{A}$ compared to data in the reconstructed energy variable. We see that $M_{A}$ changes the overall normalization of the spectrum with minimal shape variation. In Fig. 4.22 we see data compared to MC with different $\kappa$ values. $\kappa$ denotes a Pauli blocking scaling parameter which modifies the shape of the spectrum at low $Q^{2}$ while the normalization is unchanged. 


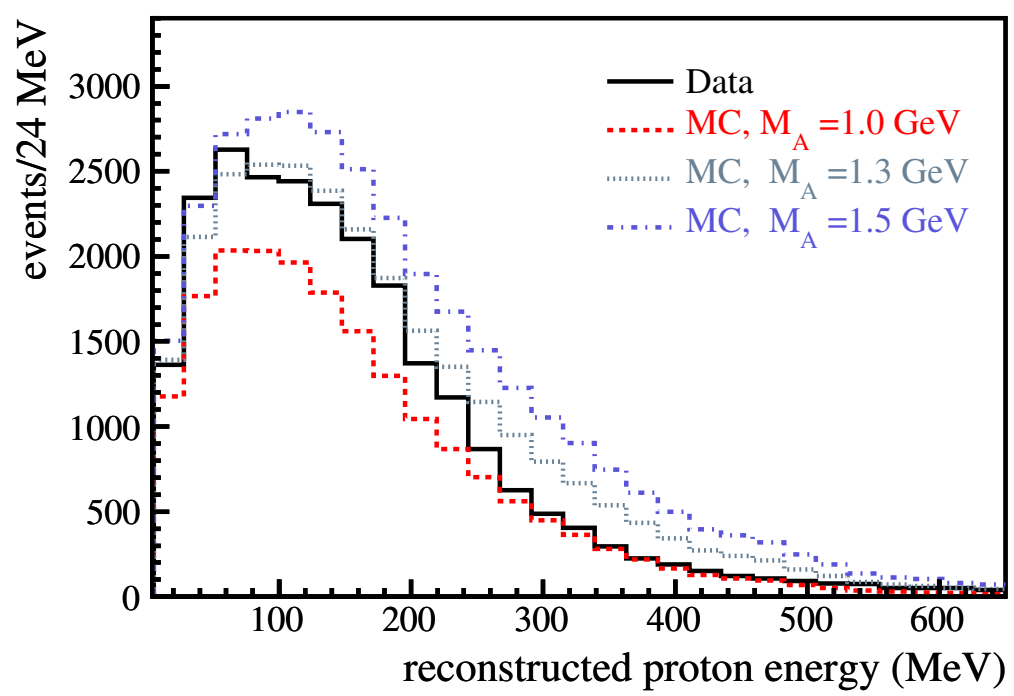

Figure 4.21: Absolutely normalized reconstructed energy spectra for antineutrino NCE data and $\mathrm{MC}$ with different $M_{A}$ values and $\kappa=1.022$.

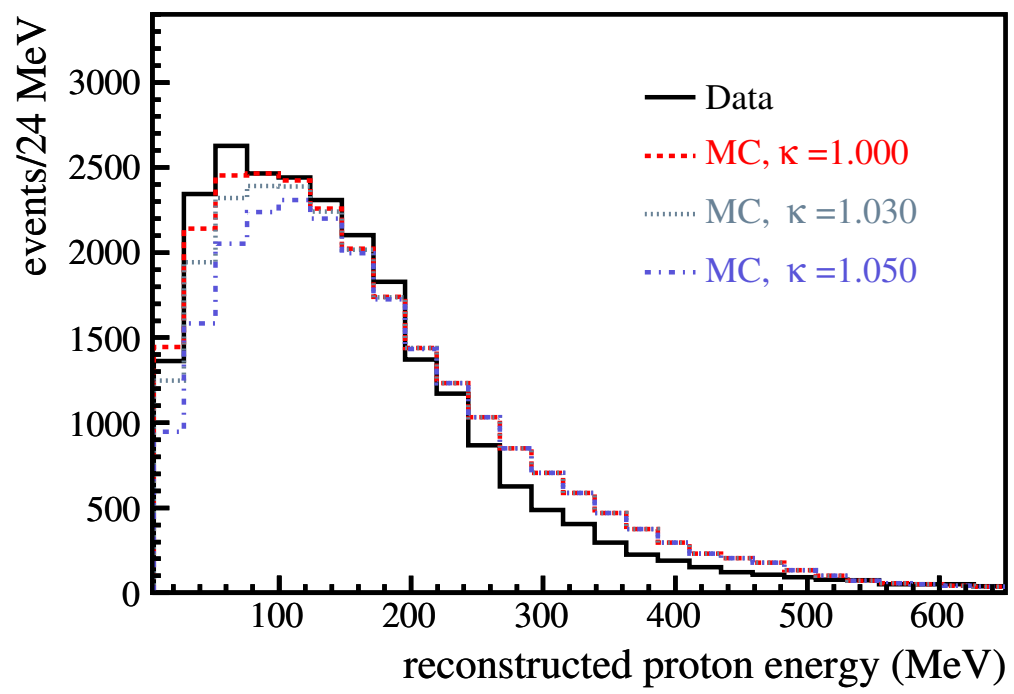

Figure 4.22: Absolutely normalized reconstructed energy spectra for antineutrino NCE data and $\mathrm{MC}$ with different $\kappa$ values and $M_{A}=1.23 \mathrm{GeV}$.

\subsubsection{Error Matrix}

To generate the error matrix which has the information of the systematic and statistical uncertainties we use the method of multisims as described before $\$ 4$.8. For each each $k$-th 
multisim we generate a value of the reconstructed energy $\mathrm{T}$ :

$$
T_{i}^{k}=D_{i} \frac{S_{i}^{k}}{S_{i}^{k}+B_{i}^{k}}
$$

where $i$ is the bin of reconstructed energy, $D$ is the data, $S$ the signal, and $B$ backgrounds. For each of the error we calculate the error matrix as:

$$
M_{i, j}=\frac{1}{N} \sum_{k=1}^{N}\left(T_{i}^{k}-T_{i}^{C V}\right)\left(T_{j}^{k}-T_{j}^{C V}\right),
$$

where $i, j=1, \ldots, n$ number of reconstructed energy bins, $N$ is the number of MC variations in the multisim, and $T^{k}$ is the $k$-th multisim and $T^{C V}$ is the $\mathrm{CV}$.

The total error matrix $\left(M_{i, j}^{\text {tot }}\right)$ is the sum of the 15 systematic and one statistical error matrices. Table 4.4 shows the list of the errors along with the normalization error for each error, which was evaluated as:

$$
\operatorname{Error}^{N}=\frac{\sqrt{\sum_{i, j} M_{i, j}}}{\int T^{C V}} .
$$

\begin{tabular}{lr} 
Source of error & value $\%$ \\
\hline \hline Beam & 3.4 \\
$\mathrm{~K}^{+}$production in the target & 0.3 \\
$\pi^{-}$production in the target & 4.7 \\
POT & 1.8 \\
Cross-section (backgrounds) & 12.0 \\
Optical Model & 18.0 \\
Dirt estimation & 1.7 \\
WS estimation & 4.9 \\
Statistical & 7.6 \\
Discriminator threshold & 0.2 \\
Charge-time PMT response & 2.9 \\
\hline Total & 24.5
\end{tabular}

Table 4.4: Individual normalization errors and the total normalization error. 


\subsubsection{Simultaneous $M_{A}$ and $\kappa$ Fit}

In order to perform a simultaneous $M_{A}$ and $\kappa$ fit to MiniBooNE data, we first generate the reconstructed MC spectrum corresponding to different combinations of $M_{A}$ and $\kappa$. For this fit we choose 54 values of $M_{A}$ from $1.00 \mathrm{GeV}$ to $1.54 \mathrm{GeV}$, and 54 values of $\kappa$ from 1.00 to 1.054. The $\chi^{2}$ is calculated as:

$$
\chi^{2}\left(M_{A}, \kappa\right)=\sum_{i=1}^{n} \sum_{j=1}^{n}\left[T_{i}^{M C}\left(M_{A}, \kappa\right)-T_{i}^{D a t a}\right]\left(M_{i, j}^{t o t}\right)^{-1}\left[T_{j}^{M C}\left(M_{A}, \kappa\right)-T_{j}^{\text {Data }}\right]
$$

where $\left(M_{i, j}^{t o t}\right)^{-1}$ is the inverse of the total error matrix evaluated in $\S 4$ 11.1. The result of the $\chi^{2}$-fit for 25 degrees of freedom is shown in Fig. 4.23 below. The best fit value of the $\chi^{2}$ corresponds to an $M_{A}=1.29 \pm 0.02 \mathrm{GeV}$ and $\kappa=1.026 \pm 0.02$.

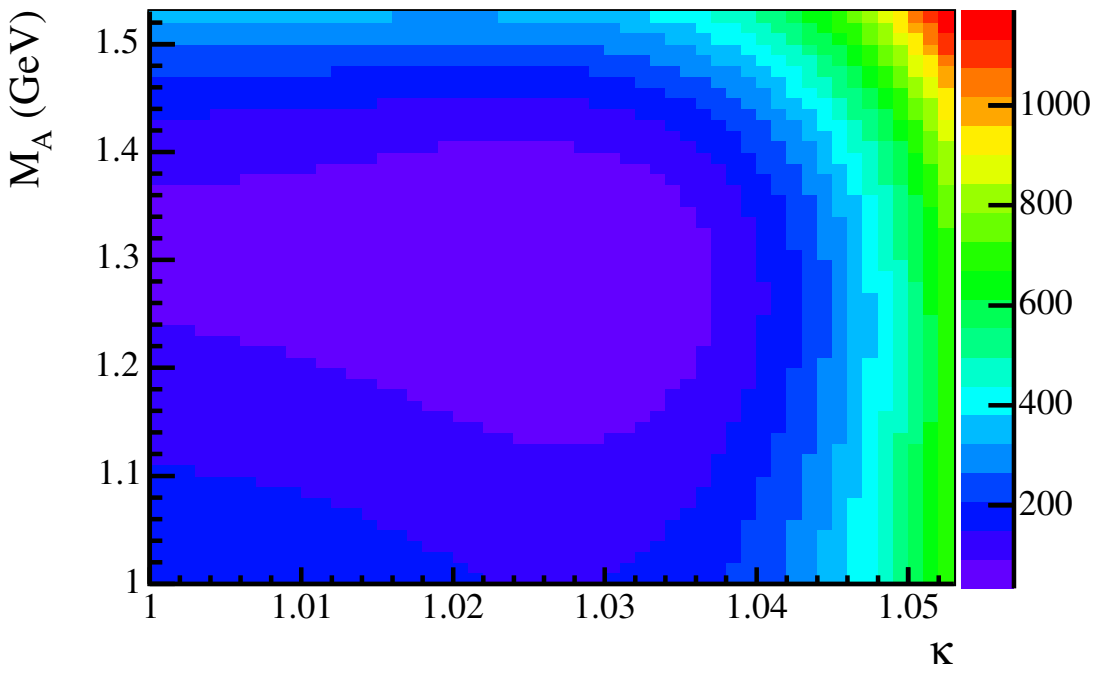

Figure 4.23: Simultaneous fit to $M_{A}$ and $\kappa$ using $\chi^{2}$ minimization. The $\chi^{2}$ values are for 25 degrees of freedom (DOF). The $\chi^{2}$ has a minimum, $\chi_{\min }^{2}=33.4$, at $M_{A}=1.29 \mathrm{GeV}$ and $\kappa=1.026$.

We also looked at the following values of $M_{A}$ and $\kappa$ which are of special interest:

- $M_{A}=1.02$ and $\kappa=1.000$, the world average value of these parameters from deuteriumbased bubble chamber experiments, 
- $M_{A}=1.39$ and $\kappa=1.000$, which was the result from the neutrino mode NCE scattering measurement [60], and

- $M_{A}=1.35$ and $\kappa=1.007$, which was the result from the neutrino mode CCQE scattering measurement [59].

Figure 4.24 shows the reconstructed energy spectrum with errors, along with the three different values of $M_{A}$ and $\kappa$, mentioned above. We see that at low energies, a higher $M_{A}$ agrees with the antineutrino NCE scattering data, however at higher energies there is disagreement between data and previous $M_{A}$ measurements in the neutrino mode. When the antineutrino NCE scattering data is compared to the world-average values of $M_{A}$ and $\kappa$, there is significant difference in normalization at low energies.

Overall we see the antineutrino NCE scattering data agrees with a high value of $M_{A}$ as compared to the world-average, which is consistent with previous measurements in the neutrino mode NCE scattering and CCQE scattering. However, the shape of the antineutrino NCE scattering spectrum is not reproduced by either the world-average value or the neutrino mode MiniBooNE measurements, which is reflected in the poor values of $\chi^{2}$ for those values of $M_{A}$ and $\kappa$. 


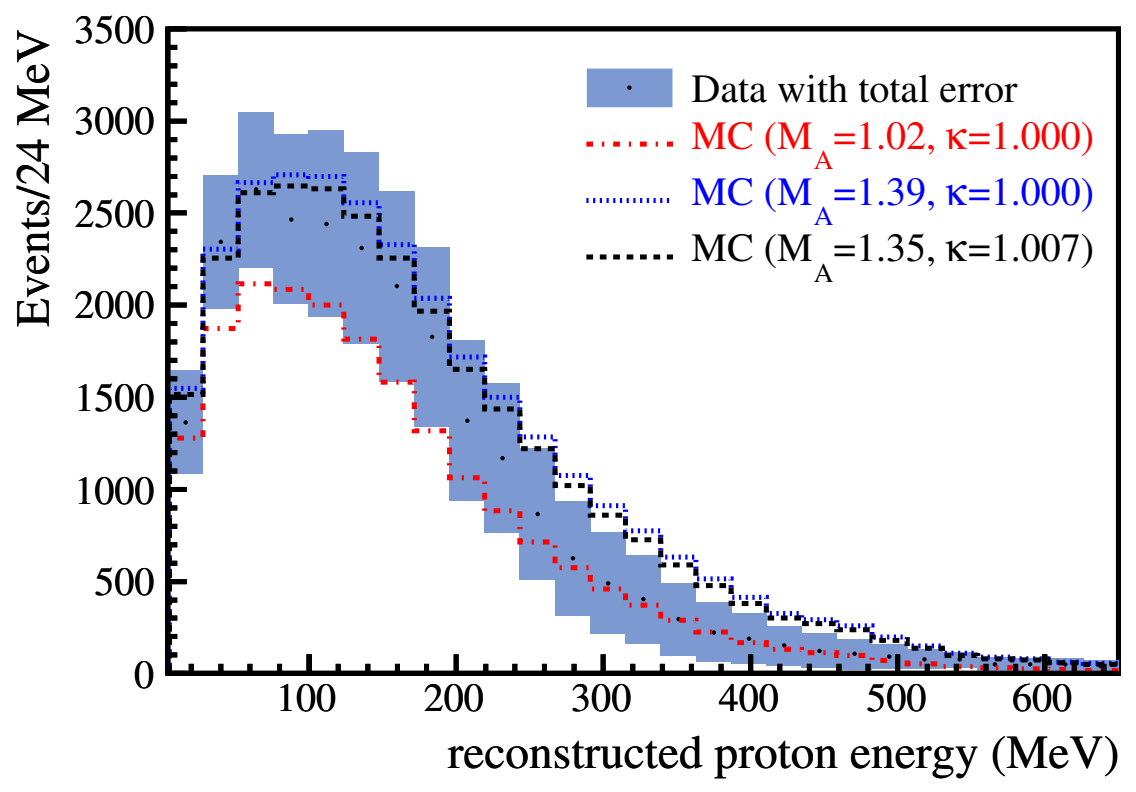

Figure 4.24: $\bar{\nu}$ NCE reconstructed energy spectrum with the total normalization error. Also shown are three different values of $M_{A}: 1.35,1.39$, and 1.02. The $\chi^{2}$ values are $78.9,107.6$, and 177.0 for 25 DOF, respectively. The distributions are absolutely normalized. 


\section{Chapter 5}

\section{Conclusions}

One of the main goals of the next-generation neutrino experiments is to test the three neutrino flavor paradigm by obtaining high-precision values of the neutrino oscillation parameters. Accelerator-based neutrino experiments play an important role in testing the oscillation model, as both the neutrino energy and oscillation length can be relatively well controlled. However, there is a dearth of neutrino (and antineutrino) cross section information in the energy region important for accelerator-based neutrino experiments [111]. The CCQE channel is an important signal interaction for the oscillation physics and there is a discrepancy between the low $(1 \mathrm{GeV})$ energy regime, reported by MiniBooNE [59] and the high (3GeV) region, as reported by NOMAD [112]. Recent results from the MiniBooNE neutrino CCQE scattering measurement and the neutrino NCE measurements point to an enhancement, with many possible theoretical explanations [40, 41, 42, 43, 44, 45].

As most of the new generation of accelerator-based neutrino detectors are made of nuclear targets such as carbon, there is an urgent need to understand the neutrino nuclear

interactions. The NCE channel provides an important cross check to the CCQE channel measurement as both have similar nuclear effects and cross section formula, furthermore NCE interactions provide information on nucleon kinematics in the experiment.

This thesis reports the antineutrino-nucleus neutral current scattering cross section (Chap- 
ter 4). The data set consists of 60,605 events passing the selection cuts corresponding to $10.1 \times 10^{20}$ POT, which represents the world's largest sample of such events. The final sample is more than one order of magnitude lager that the previous antineutrino NCE scattering cross section measurement reported by the BNL E734 experiment. Furthermore, the measurement presented in this dissertation spans a wider range in $Q^{2}$, including the low- $Q^{2}$ regime where the cross section rollover is clearly visible.

A $\chi^{2}$-based minimization was performed to determine the best value of $M_{A}$ and $\kappa$ that matches the antineutrino NCE scattering data. However, the best fit values of $M_{A}=1.29 \mathrm{GeV}$ and $\kappa=1.026$ still give a relatively poor $\chi^{2}$, which suggests that the underlying nuclear model (based largely on the relativistic Fermi gas model) may not be an accurate representation for this particular interaction.

Additionally, we present a measurement of the antineutrino/neutrino-nucleus NCE scattering cross section ratio. The neutrino mode NCE sample used in this study, corresponding to $6.4 \times 10^{20} \mathrm{POT}$, is also the world's largest sample (also by an order of magnitude). We have demonstrated that the ratio measurement is robust, as most of the correlated errors cancel, as expected. Furthermore, this ratio also proves to be rather insensitive to variations in the axial mass and the Pauli blocking parameter. This is the first time that this ratio has been experimentally reported. We believe this measurement will aid the theoretical physics community to test various model predictions of neutrino-nucleon/nucleus interactions.

In Appendix A, we discuss a possibility of searching for a light ( $\mathrm{MeV}$-scale) dark matter candidate events which can appear in the neutral-current nucleon scattering sample. Currently, most searches for dark matter are looking for a weakly interacting massive particle (WIMP) of GeV-scale mass. However, a MeV-scale dark matter particle is theoretically equally probable. 


\section{Appendix A}

\section{Search for a light dark matter particle at MiniBooNE}

\section{A.1 Introduction}

Recently there have been a number of publications exploring the sensitivity of fixed-target neutrino experiments to $\mathrm{MeV}$-scale dark matter candidates $[113,114]$. Many of these models look for non-gravitational interaction signatures which are of particular interest to particle physics. Light dark matter candidates or mediator particles, that annihilate, scatter or decay, have been explored to explain anomalous experimental observations. [115, 116].

An interesting consequence of models with light (sub-GeV) dark matter is the production of high intensity "dark matter beam", generated as dark matter particles are pair-produced as a result proton-target interactions and boosted along the beam direction. The ensuing scattering of these light dark matter would manifest as an excess over the known neutralcurrent type scattering events. 

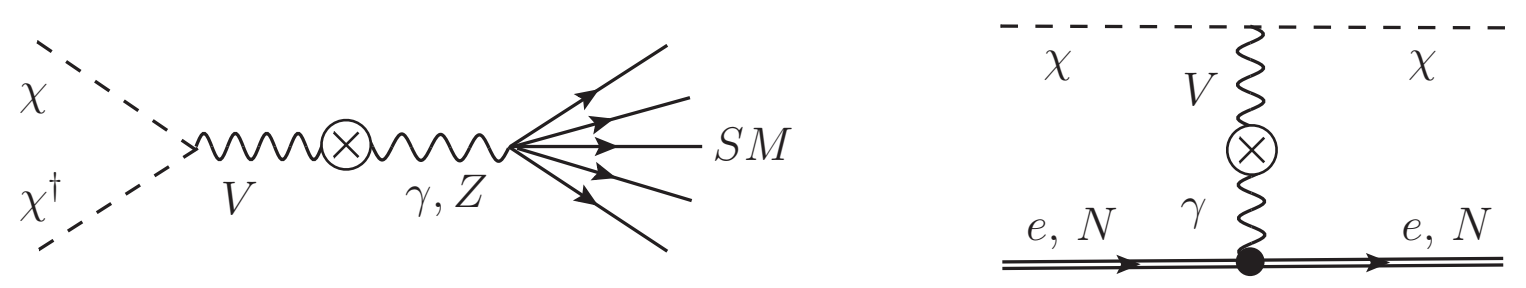

Figure A.1: Tree-level annihilation (left) and scattering (right) of scalar dark matter in the $\mathrm{U}(1)^{\prime}$ hidden sector.

\section{A.2 MiniBooNE Neutral Current Interactions}

A recent paper [117] is of particular interest. In this analysis the authors explore a class of MeV-scale dark matter models, originally used to explain the strength of $511 \mathrm{keV}$ emission from the galactic center [118].

The authors employ a modified Lagrangian:

$$
\mathcal{L}_{V, \chi}=-\frac{1}{4} V_{\mu \nu}^{2}+\frac{1}{2} m_{v}^{2} V_{\mu}^{2}+\kappa V_{\mu} \partial_{\mu} F^{\nu \mu}+\left|D_{\mu} \chi\right|^{2}-m_{\chi}^{2}|\chi|^{2}+\mathcal{L}_{h^{\prime}}
$$

which assumes a hidden sector charged under a U(1)' gauge group, with a vector portal coupling to the SM via kinetic mixing [119], which is spontaneously broken at a low scale by a Higgs' sector leading to a mass for the vector mediator $V_{\mu}$. The model has four parameters: $m_{\chi}, m_{V}$-the masses of the dark matter candidate and its vector mediator, the $\mathrm{U}(1)$ ' gauge coupling e', and the kinetic mixing coefficient $\kappa$.

The annihilation of the dark matter candidate $\chi$ is given by the diagram in Fig.A.1 (left). Rotating the diagram gives and corresponding scattering rate off electrons and nucleons in Fig.A.1 (right) which can, in principle, be used to detect the dark matter candidate. A dark matter beam can be produced via the following processes (as taken from Ref. [117], where some of the emerging particles have been suppressed), for a small $m_{V}$ :

1. $p+p \rightarrow X+\pi^{0}, \eta$

2. $\pi^{0}, \eta \rightarrow \gamma+V$ 


\section{3. $V \rightarrow 2 \chi$}

and may be detected through neutral current-like processes with electrons or nucleons:

1. $e+\chi \rightarrow e+\chi$

2. $N+\chi \rightarrow N+\chi$

By calculating the number of dark matter neutral current-like scattering events that would be expected at MiniBooNE and comparing it to the actual number, from the neutrinonucleon data [60], and estimating the number of neutrino-electron scattering events, the authors were able to probe the parameter space of this model. The sensitivity is limited due to the fact that the authors had to estimate the rate of electron scattering in MiniBooNE which has not been published. The results are shown in Fig.4.

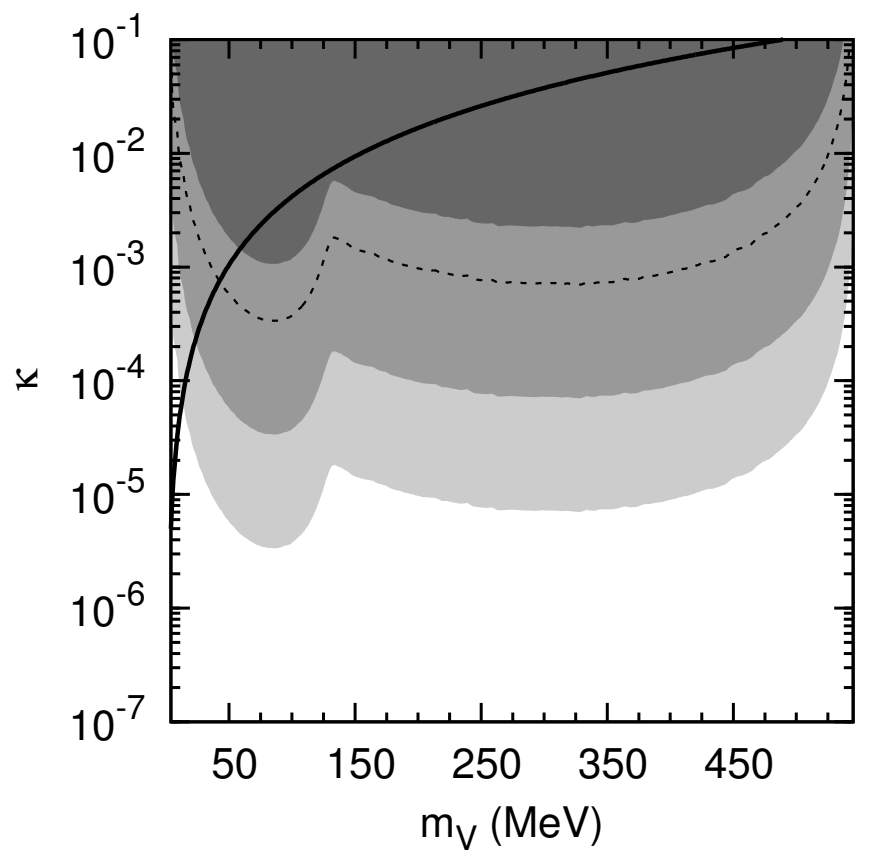

Figure A.2: Expected number of neutral current-like dark matter nucleon scattering events at MiniBooNE. The regions represent greater than 10 (light), 1000 (medium) and $10^{6}$ (dark) expected events for $m_{\chi}=1 \mathrm{MeV}$. The black line corresponds to a particular value of $\kappa$ and the dotted line indicates the total number of neutrino events observed. Figure from Ref. [117] 


\section{A.3 Plan of the Project}

We propose to do a search for light $(\sim 1 \mathrm{MeV}$ scale) dark matter at the MiniBooNE experiment. The analysis will be along similar lines as discussed in §A.2.

First we will calculate the number dark matter of neutral current-like scattering events expected at MiniBooNE detector, following [117] and compare it to the total number of such events actually observed. The sample would include:

1. all neutrino-nucleus and antineutrino-nucleus scattering data collected by MiniBooNE up to May 2011.

2. hitherto unpublished electron scattering data, again both neutrino-electron and antineutrinoelectron scattering data accumulated in the same run period as above. (MiniBooNE has the ability to identify electron scattering events, see [120] for details)

We believe that by looking at a larger sample of neutral current-like events and having access to accurate detector efficiency information, we will be able to probe a larger region of the parameter space described in this model.

\section{Possibility of using time of flight}

Assuming that some neutral current scattering events are due to massive dark matter particles as opposed to (almost) massless neutrinos, there will be a time difference between such scattering events and those caused by neutrinos. The (more) massive dark matter candidates would likely cause scattering events after the neutrino beam has arrived at the detector.

MiniBooNE employs a resistive wall monitoring (RWM) system which gives us accurate information of beam arrival time to the detector. We propose to exploit the excellent timing

resolution of the MiniBooNE detector $(\sim 2 \mathrm{~ns})$ to look for any potential accompanying dark matter beam by looking for late events within the beam window. See [70] for a description of the timing resolution of MiniBooNE detector. 


\section{A.4 Conclusion}

The proposed dark matter search in MiniBooNE opens an exciting experimental avenue to search for the hidden sector. It would also help in excluding exotic models as we look for beyond standard model physics. 


\section{References}

[1] J. Beringer et al., "Review of particle physics," Phys. Rev., vol. D86, p. 010001, 2012.

[2] S. Chatrchyan et al., "Observation of a new boson at a mass of $125 \mathrm{GeV}$ with the CMS experiment at the LHC," 2012.

[3] G. Aad et al., "Observation of a new particle in the search for the Standard Model Higgs boson with the ATLAS detector at the LHC," 2012.

[4] W. Pauli, "Dear radioactive ladies and gentlemen," Phys. Today, vol. 31N9, p. 27, 1978.

[5] E. Fermi, "Versuch einer Theorie der $\beta$-Strahlen. 1," Z. Phys., vol. 88, pp. 161-177, 1934.

[6] T. D. Lee and C.-N. Yang, "Question of Parity Conservation in Weak Interactions," Phys. Rev., vol. 104, pp. 254-258, 1956.

[7] C. S. Wu, E. Ambler, R. W. Hayward, D. D. Hoppes, and R. P. Hudson, "Experimental test of parity conservation in beta decay," Phys. Rev., vol. 105, pp. 1413-1414, 1957.

[8] E. C. G. Sudarshan and R. E. Marshak, "Chirality invariance and the universal Fermi interaction," Phys. Rev., vol. 109, pp. 1860-1860, 1958.

[9] R. P. Feynman and M. Gell-Mann, "Theory of the Fermi interaction," Phys. Rev., vol. 109, pp. 193-198, 1958.

[10] C. L. Cowan, F. Reines, F. B. Harrison, H. W. Kruse, and A. D. McGuire, "Detection of the free neutrino: A Confirmation," Science, vol. 124, pp. 103-104, 1956.

[11] G. Danby, J. Gaillard, K. A. Goulianos, L. Lederman, N. B. Mistry, et al., "Observation of High-Energy Neutrino Reactions and the Existence of Two Kinds of Neutrinos," Phys.Rev.Lett., vol. 9, pp. 36-44, 1962. 
[12] S. L. Glashow, "Partial Symmetries of Weak Interactions," Nucl. Phys., vol. 22, pp. 579-588, 1961.

[13] S. Weinberg, "A Model of Leptons," Phys. Rev. Lett., vol. 19, pp. 1264-1266, 1967.

[14] A. Salam and J. C. Ward, "Weak and electromagnetic interactions," Nuovo Cim., vol. 11, pp. 568-577, 1959.

[15] P. W. Higgs, "Broken Symmetries And The Masses Of Gauge Bosons," Phys. Rev. Lett., vol. 13, pp. 508-509, 1964.

[16] F. J. Hasert et al., "Observation of neutrino-like interactions without muon or electron in the Gargamelle neutrino experiment," Phys. Lett., vol. B46, pp. 138-140, 1973.

[17] A. Astbury, "Found: W and Z. The discovery of the W and Z intermediate vector bosons at CERN's proton anti-proton collider," Phys.Bull., vol. 34, pp. 434-436, 1983.

[18] C. Quigg, Gauge Theories Of Strong, Weak, And Electromagnetic Interactions (Advanced Book Classics). Westview Press, 1997.

[19] C. Quigg, "Nature's Greatest Puzzles," eConf, vol. C040802, p. L001, 2004.

[20] V. M. Abazov et al., "Measurement of the anomalous like-sign dimuon charge asymmetry with $9 \mathrm{fb}^{-} 1$ of p pbar collisions," Phys.Rev., vol. D84, p. 052007, 2011. Submitted to Phys. Rev. D.

[21] G. L. Fogli, E. Lisi, D. Montanino, and G. Scioscia, "Neutrino oscillations. A Phenomenological overview," Nucl.Phys.Proc.Suppl., vol. 66, pp. 315-334, 1998.

[22] J. Davis, Raymond, D. S. Harmer, and K. C. Hoffman, "Search for neutrinos from the sun," Phys.Rev.Lett., vol. 20, pp. 1205-1209, 1968.

[23] J. N. Bahcall, "Solar neutrinos. I: Theoretical," Phys.Rev.Lett., vol. 12, pp. 300-302, 1964.

[24] K. Hirata et al., "Experimental Study of the Atmospheric Neutrino Flux," Phys.Lett., vol. B205, p. 416, 1988.

[25] Y. Fukuda et al., "Measurement of a small atmospheric muon-neutrino / electronneutrino ratio," Phys.Lett., vol. B433, pp. 9-18, 1998. 
[26] Q. Ahmad et al., "Direct evidence for neutrino flavor transformation from neutral current interactions in the Sudbury Neutrino Observatory," Phys.Rev.Lett., vol. 89, p. 011301, 2002.

[27] M. Ahn et al., "Measurement of Neutrino Oscillation by the K2K Experiment," Phys.Rev., vol. D74, p. 072003, 2006.

[28] D. Michael et al., "Observation of muon neutrino disappearance with the MINOS detectors and the NuMI neutrino beam," Phys.Rev.Lett., vol. 97, p. 191801, 2006.

[29] Y. Abe et al., "Reactor electron antineutrino disappearance in the Double Chooz experiment," 2012.

[30] J. Ahn et al., "Observation of Reactor Electron Antineutrino Disappearance in the RENO Experiment," Phys.Rev.Lett., vol. 108, p. 191802, 2012.

[31] F. An et al., "Observation of electron-antineutrino disappearance at Daya Bay," Phys.Rev.Lett., vol. 108, p. 171803, 2012.

[32] V. Lobashev et al., "Direct search for neutrino mass and anomaly in the tritium beta-spectrum: Status of 'Troitsk neutrino mass' experiment," Nucl.Phys.Proc.Suppl., vol. 91, pp. 280-286, 2001.

[33] D. N. Spergel et al., "First year wilkinson microwave anisotropy probe (wmap) observations: Determination of cosmological parameters," The Astrophysical Journal Supplement Series, vol. 148, no. 1, p. 175, 2003.

[34] P. A. M. Dirac, "A theory of electrons and protons," Proceedings of the Royal Society of London. Series A, vol. 126, no. 801, pp. 360-365, 1930.

[35] W. Pauli and V. Weisskopf Helv. Phys. Acta., vol. 7, 1934.

[36] C. D. Anderson, "The Positive Electron," Physical Review, vol. 43, pp. 491-494, Mar. 1933.

[37] E. Stueckelberg, "The theory of positrons," Helv. Phys. Acta., vol. 15, pp. 51-80, 1942.

[38] R. P. Feynman, "The theory of positrons," Phys. Rev., vol. 76, pp. 749-759, Sep 1949.

[39] E. Majorana, "Teoria simmetrica dell'elettrone e del positrone (in Italian)," Nuovo Cimento, vol. 14, p. 171, 1937. 
[40] M. Martini, M. Ericson, and G. Chanfray, "Neutrino quasielastic interaction and nuclear dynamics," Phys.Rev., vol. C84, p. 055502, 2011.

[41] J. Nieves, I. R. Simo, and M. J. V. Vacas, "Inclusive charged-current neutrino-nucleus reactions," Phys. Rev. C, vol. 83, p. 045501, Apr 2011.

[42] J. Amaro, M. Barbaro, J. Caballero, and T. Donnelly, "Meson-exchange currents and quasielastic antineutrino cross sections in the SuperScaling Approximation," Phys.Rev.Lett., vol. 108, p. 152501, 2012.

[43] O. Benhar and G. Veneziano, "Nuclear effects in neutral current quasi-elastic neutrino interactions," Phys.Lett., vol. B702, pp. 433-437, 2011.

[44] A. Meucci, C. Giusti, and F. D. Pacati, "Relativistic descriptions of final-state interactions in neutral-current neutrino-nucleus scattering at MiniBooNE kinematics," Phys.Rev., vol. D84, p. 113003, 2011.

[45] A. M. Ankowski, O. Benhar, and N. Farina, "Analysis of the $Q^{2}$-dependence of chargedcurrent quasielastic processes in neutrino-nucleus interactions," Phys.Rev., vol. D82, p. 013002, 2010.

[46] A. W. Thomas and W. Weise, The Structure of the Nucleon. Wiley-VCH, 2001.

[47] E. Rutherford, "The Scattering of $\alpha$ and $\beta$ Particles by Matter and the Structure of the Atom," Philos. Mag., vol. 6, p. 21, 1911.

[48] N. Mott Proc. Roy. Soc. London, vol. A 124/A 135, p. 425/429, 1929/1932.

[49] N. Mott Proc. Roy. Soc. London, vol. A 135, p. 429, 1932.

[50] O. Stern Nature, vol. 132, p. 103, 1933.

[51] E. E. Chambers and R. Hofstadter, "Structure of the proton," Phys. Rev., vol. 103, pp. 1454-1463, Sep 1956.

[52] M. N. Rosenbluth Phys. Rev., vol. 79, 1950.

[53] M. R. Yearian and R. Hofstader Phys. Rev., vol. 110, p. 552, 1958.

[54] V. Punjabi, C. Perdrisat, K. Aniol, F. Baker, J. Berthot, et al., "Proton elastic formfactor ratios to $Q^{2}=3.5-G_{e} V^{2}$ by polarization transfer," Phys.Rev., vol. C71, p. $055202,2005$. 
[55] B. Aubert, A. Benvenuti, D. Cline, W. T. Ford, R. Imlay, et al., "Further Observation of Muonless Neutrino Induced Inelastic Interactions," Phys.Rev.Lett., vol. 32, pp. 1454$1457,1974$.

[56] J. E. Kim, P. Langacker, M. Levine, and H. H. Williams, "A theoretical and experimental review of the weak neutral current: a determination of its structure and limits on deviations from the minimal $\mathrm{SU}(2)_{L} \times \mathrm{U}(1)$ electroweak theory," Rev. Mod. Phys., vol. 53, pp. 211-252, Apr 1981.

[57] J. Ashman et al., "A Measurement of the Spin Asymmetry and Determination of the Structure Function g(1) in Deep Inelastic Muon-Proton Scattering," Phys.Lett., vol. B206, p. 364, 1988.

[58] E. A. Paschos, Electroweak Theory. Cambridge University Press, 2007.

[59] A. Aguilar-Arevalo et al., "First Measurement of the Muon Neutrino Charged Current Quasielastic Double Differential Cross Section," Phys.Rev., vol. D81, p. 092005, 2010.

[60] A. Aguilar-Arevalo et al., "Measurement of the Neutrino Neutral-Current Elastic Differential Cross Section on Mineral Oil at $E_{\nu} \sim 1 \mathrm{GeV}$," Phys.Rev., vol. D82, p. 092005, 2010.

[61] C. Llewellyn Smith, "Neutrino Reactions at Accelerator Energies," Phys.Rept., vol. 3, pp. 261-379, 1972.

[62] L. Ahrens, S. Aronson, P. Connolly, B. Gibbard, M. Murtagh, et al., "Measurement of Neutrino - Proton and anti-neutrino - Proton Elastic Scattering," Phys.Rev., vol. D35, p. $785,1987$.

[63] G. Garvey, E. Kolbe, K. Langanke, and S. Krewald Phys. Rev., vol. C48, no. 1919, 1993.

[64] E. Church et al., "A letter of intent for an experiment to measure $\nu_{\mu} \rightarrow \nu_{e}$ oscillations and $\mathrm{nu} / \mathrm{mu}$ disappearance at the Fermilab booster: BooNE," 1997.

[65] C. Athanassopoulos et al., "Evidence for $\nu_{\mu} \rightarrow \nu_{e}$ neutrino oscillations from LSND," Phys. Rev. Lett., vol. 81, pp. 1774-1777, 1998.

[66] C. Athanassopoulos et al., "Evidence for $\nu_{\mu} \rightarrow \nu_{e}$ neutrino oscillations from the LSND experiment at LAMPF," Phys.Rev.Lett., vol. 77, pp. 3082-3085, 1996. 
[67] A. Aguilar-Arevalo et al., "A Search for electron neutrino appearance at the $\Delta m^{2} \sim$ 1eV² scale," Phys.Rev.Lett., vol. 98, p. 231801, 2007.

[68] A. Aguilar-Arevalo et al., "A Search for Electron Antineutrino Appearance at the Delta m**2 1-eV**2 Scale," Phys.Rev.Lett., vol. 103, p. 111801, 2009.

[69] A. Aguilar-Arevalo et al., "Event Excess in the MiniBooNE Search for $\bar{\nu}_{\mu} \rightarrow \bar{\nu}_{e}$ Oscillations," Phys.Rev.Lett., vol. 105, p. 181801, 2010.

[70] A. Aguilar-Arevalo et al., "The MiniBooNE Detector," Nucl.Instrum.Meth., vol. A599, pp. 28-46, 2009.

[71] I. Stancu et al., "Technical Design Report for the MiniBooNE Neutrino Beam," 2001.

[72] B. Engineering, "http://www.bartoszekeng.com/mboone/mboone.htm.,"

[73] S. Agostinelli et al., "GEANT4: A simulation toolkit," Nucl. Instrum. Meth., vol. A506, pp. 250-303, 2003.

[74] D. C. Carey, K. Brown, and F. Rothacker, "Third order TRANSPORT with MAD input: A Computer program for designing charged particle beam transport systems," 1998.

[75] R. Glauber and G. Matthiae, "High-energy scattering of protons by nuclei," Nucl.Phys., vol. B21, pp. 135-157, 1970.

[76] K. J. Foley et al., "Small-Angle Elastic Scattering of Protons and Pions, 7-20 BeV/c," Phys. Rev. Lett., vol. 11, pp. 425-429, 1963.

[77] R. M. Barnett et al., "(particle data group)," Phys. Rev., vol. D54, 1996.

[78] A. A. Aguilar-Arevalo et al., "The Neutrino Flux prediction at MiniBooNE," Phys. Rev., vol. D79, p. 072002, 2009.

[79] M. G. Catanesi et al., "Measurement of the production cross-section of positive pions in the collision of $8.9 \mathrm{GeV} / \mathrm{c}$ protons on beryllium," Eur. Phys. J., vol. C52, pp. 29-53, 2007.

[80] J. R. Stanford and C. L. Wang BNL Note 11299, 1967.

[81] I. Chemakin et al., "Pion Production by Protons on a Thin Beryllium Target at 6.4, 12.3, and $17.5 \mathrm{GeV} / \mathrm{c}$ Incident Proton Momenta," Phys. Rev., vol. C77, p. 015209, 2008. 
[82] T. Abbott et al., "Measurement of particle production in proton induced reactions at 14.6-GeV/c," Phys. Rev., vol. D45, pp. 3906-3920, 1992.

[83] I. A. Aleshin, Y. D. Drabkin and V. V. Kolesnikov, "Production of KÂś Mesons from Be Targets at 62-Mrad at 9.5-GeV/c Incident Proton Momenta," ITEP, Moscow, 1977.

[84] F. Abe et al., "Production of neutral strange particles $K^{0}(S)$ and $\Lambda_{0}$ by 12-GeV protons on nuclear targets," Phys. Rev., vol. D36, pp. 1302-1319, 1987.

[85] N. V. Mokhov et al., "MARS Code Developments," 1998.

[86] A. Ferrari, P. R. Sala, A. Fasso, and J. Ranf, "FLUKA: A multi-particle transport code (Program version 2005)," cERN-2005-010.

[87] A. Aguilar-Arevalo et al., "The miniboone detector," Nuclear Instruments and Methods in Physics Research Section A: Accelerators, Spectrometers, Detectors and Associated Equipment, vol. 599, no. 1, pp. 28 - 46, 2009.

[88] H. Meyer, "Index of Refraction of Marcol 7," MiniBooNE Techincal Note, no. TN 190.

[89] J. Raaf, E. Hawker, R. Johnson, A. Bazarko, G. Garvey, et al., "Mineral oil tests for the MiniBooNE detector," IEEE Trans.Nucl.Sci., vol. 49, pp. 957-962, 2002.

[90] S. Brice et al., "Photomultiplier tubes in the MiniBooNE experiment," Nucl.Instrum.Meth., vol. A562, pp. 97-109, 2006.

[91] B. T. Fleming, L. Bugel, E. Hawker, V. Sandberg, S. Koutsoliotas, et al., "Photomultiplier tube testing for the MiniBooNE experiment," IEEE Trans.Nucl.Sci., vol. 49, pp. 984-988, 2002.

[92] R. Imlay, B. Metcalf, S. Ouedraogo, M. Sung, and M. Wascko, "Energy Calibration of Stopping Muons in MiniBooNE Using the Muon Tracker and Cubes," MiniBooNE Techincal Note, no. TN 106.

[93] R. B. Patterson, "A search for muon neutrino to electron neutrino oscillations at $\Delta m^{2}>0.1 e V^{2}, " 2007$. URA Thesis Award 2008.

[94] D. Casper, "The Nuance neutrino physics simulation, and the future," Nucl.Phys.Proc.Suppl., vol. 112, pp. 161-170, 2002. To be published in Proceedings of the First International Workshop on Neutrino-Nucleus Interactions in the Few-GeV Region (NUINT'01). 
[95] R. A. Smith and E. J. Moniz, "Neutrino reactions on nuclear targets," Nucl. Phys., vol. B43, p. 605, 1972 .

[96] H. S. Budd, A. Bodek, and J. Arrington, "Modeling quasielastic form-factors for electron and neutrino scattering," 2003.

[97] D. Rein and L. M. Sehgal, "Coherent pi0 Production in Neutrino Reactions," Nucl.Phys., vol. B223, p. 29, 1983.

[98] A. A. Aguilar-Arevalo et al., "Measurement of nu(mu) and anti-nu(mu) induced neutral current single pi0 production cross sections on mineral oil at E(nu) O(1- GeV)," Phys.Rev., vol. D81, p. 013005, 2010.

[99] T. Katori, "A Measurement of the Muon Neutrino Carged Current Quasielastic Interaction and a Test of LorentZ Violation with the MiniBooNE Experiment," 2008. Ph.D. thesis.

[100] R. Brun, F. Bruyant, M. Maire, A. McPherson, and P. Zanarini, "GEANT3," 1987. Revised version.

[101] D. C. Cox, "A measurement of the neutral current neutrino-nucleon elastic cross section at MiniBooNE," 2008. Ph.D. Thesis.

[102] C. Zeitnitz and T. A. Gabriel, "The GEANT - CALOR interface and benchmark calculations of ZEUS test calorimeters," Nucl. Instrum. Meth., vol. A349, pp. 106-111, 1994.

[103] A. Bazarko et al., "Studies of Scattering in Marcol 7 Mineral Oil," MiniBooNE Techincal Note, no. TN 144.

[104] D. Toptygin, "Time-Resolved Fluorescence of MiniBooNE Mineral Oil," MiniBooNE Techincal Note, no. 122.

[105] D. Perevalov, "Neutrino-nucleus neutral current elastic interactions measurement in MiniBooNE," 2009. Ph.D. Thesis (advisor: Ion Stancu).

[106] R. Patterson, E. Laird, Y. Liu, P. Meyers, I. Stancu, and H. A. Tanaka, "The Extendedtrack reconstruction for MiniBooNE," Nucl.Instrum.Meth., vol. A608, pp. 206-224, 2009.

[107] A. Aguilar-Arevalo et al., "Measurement of the neutrino component of an anti-neutrino beam observed by a non-magnetized detector," Phys.Rev., vol. D84, p. 072005, 2011. 
[108] J. Grange, "New Results from MiniBooNE Charged-Current Quasi-Elastic AntiNeutrino Data," AIP Conf.Proc., vol. 1405, pp. 83-88, 2011.

[109] T. Suzuki, D. F. Measday, and J. Roalsvig, "Total Nuclear Capture Rates for Negative Muons," Phys.Rev., vol. C35, p. 2212, 1987.

[110] G. Cowan, "Statistics," Phys. Lett., vol. B667, p. 1, 2008.

[111] J. Hewett, H. Weerts, R. Brock, J. Butler, B. Casey, et al., "Fundamental Physics at the Intensity Frontier," 2012.

[112] V. Lyubushkin et al., "A Study of quasi-elastic muon neutrino and antineutrino scattering in the NOMAD experiment," Eur.Phys.J., vol. C63, pp. 355-381, 2009.

[113] J. D. Bjorken, R. Essig, P. Schuster, and N. Toro, "New Fixed-Target Experiments to Search for Dark Gauge Forces," Phys.Rev., vol. D80, p. 075018, 2009.

[114] B. Batell, M. Pospelov, and A. Ritz, "Exploring Portals to a Hidden Sector Through Fixed Targets," Phys.Rev., vol. D80, p. 095024, 2009.

[115] D. Hooper and K. M. Zurek, "A Natural Supersymmetric Model with MeV Dark Matter," Phys.Rev., vol. D77, p. 087302, 2008.

[116] N. Arkani-Hamed, D. P. Finkbeiner, T. R. Slatyer, and N. Weiner, "A Theory of Dark Matter," Phys.Rev., vol. D79, p. 015014, 2009.

[117] P. deNiverville, M. Pospelov, and A. Ritz, "Observing a light dark matter beam with neutrino experiments," Phys.Rev., vol. D84, p. 075020, 2011.

[118] C. Boehm, D. Hooper, J. Silk, M. Casse, and J. Paul, "MeV dark matter: Has it been detected?," Phys.Rev.Lett., vol. 92, p. 101301, 2004.

[119] M. Pospelov and A. Ritz, "Astrophysical Signatures of Secluded Dark Matter," Phys.Lett., vol. B671, pp. 391-397, 2009.

[120] S. A. Ouedraogo, "Limit on the muon neutrino magnetic moment and a measurement of the CCPIP to CCQE cross section ratio," 2008. Ph.D. Thesis (advisors: William J. Metcalf, Richard L. Imlay). 